\title{
Assessment of the allocation of HIV funding in Indonesia
}

Richard T. Gray ${ }^{1}$, Josephine Reyes ${ }^{1}$, Mardiati Nadjib², Pandu Harimurti ${ }^{3}$, David P. Wilson ${ }^{1}$

October 2012

${ }^{1}$ The Kirby Institute, University of New South Wales, Sydney, Australia

${ }^{2}$ School of Public Health, University of Indonesia

${ }^{3}$ World Bank Group, Jakarta, Indonesia

\section{Table of contents}

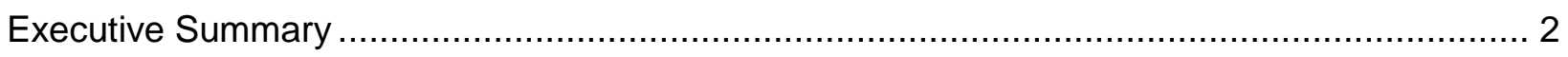

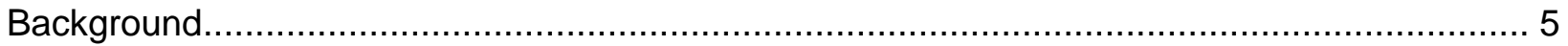

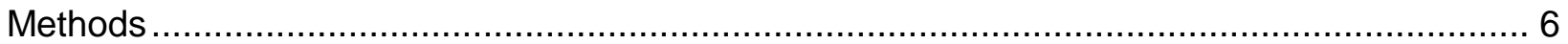

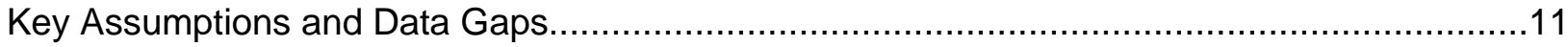

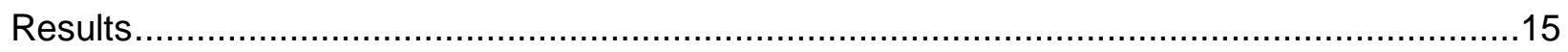

Appendix 1: HIV Spending Data Collation, Estimation of Costs......................................26

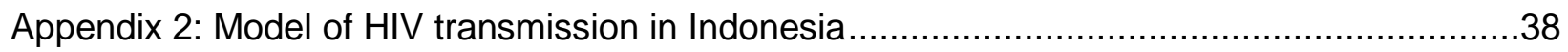

Appendix 3: Calibration of model to the HIV epidemics in non-Papua and Papua ....................40

Appendix 4: Relationships between HIV/AIDS Spending and Behavioral Parameters..............44

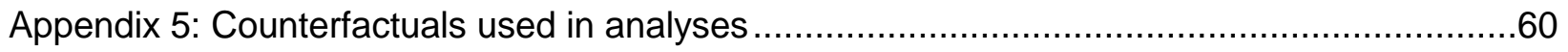

Appendix 6: Optimal Allocation of Future HIV Funding ...............................................72

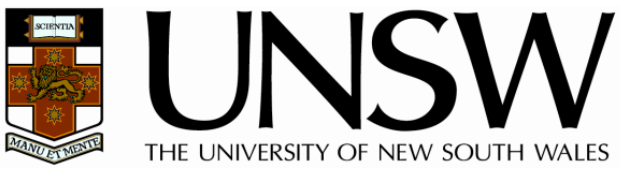


(C) International Bank for Reconstruction and Development / The World Bank

1818 H Street NW, Washington DC 20433

Internet: www.worldbank.org; Telephone: 2024731000

This work is a product of the staff of The World Bank. The findings, interpretations, and conclusions expressed in this work do not necessarily reflect the views of the Executive Directors of The World Bank or other partner institutions or the governments they represent. The World Bank does not guarantee the accuracy of the data included in this work. The boundaries, colors, denominations, and other information shown on any map in this work do not imply any judgment on the part of The World Bank concerning the legal status of any territory or the endorsement or acceptance of such boundaries.

Nothing herein shall constitute or be considered to be a limitation upon or waiver of the privileges and immunities of The World Bank, all of which are specifically reserved.

\section{Rights and Permissions}

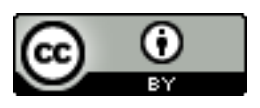

This work is available under the Creative Commons Attribution 3.0 Unported licence (CC BY 3.0) http://creativecommons.org/licences/by/3.0. Under the Creative Commons Attribution license, you are free to copy, distribute and adapt this work, including for commercial purposes, under the following conditions:

Attribution - Please cite the work as follows: The World Bank. 2018. Assessment of the allocation of HIV funding in Indonesia. Washington DC: World Bank. License: Creative Commons Attribution CC BY 3.0

Translations - If you create a translation of this work, please add the following disclaimer along with the attribution: This translation was not created by The World Bank and should not be considered an official World Bank translation. The World Bank shall not be liable for any content or error in its translation.

All queries on rights and licenses should be addressed to the Office of the Publisher, The World Bank, 1818 H Street NW, Washington DC, 20433, USA; fax: 202-522-2625; email:

pubrights@worldbank.org. 


\section{Executive Summary}

This study aimed to estimate the cost-effectiveness and return on investment of HIV programs implemented during 2003-2010 in Indonesia and to contribute to the allocative efficiency of the HIV prevention response in Indonesia through analyses to inform the prioritization of resources for the 2015-2019 national HIV budget in the context of reductions in international donor funding. This study aims to establish evidence of cost-effectiveness and optimal allocations of limited resources for greatest epidemiological impact. Costs of programs were ascertained through review of National AIDS Spending Assessments, an Indonesian costing for harm reduction study, and utilization of unit cost calculations. Relationships between program spending over time and program-targeted risk factors or other relevant end points were assessed and incorporated into a mathematical epidemiological HIV model of Papua/non-Papua Indonesia. The spending-outcome relationships and epidemiological model were used to compare observed conditions with counterfactual scenarios of reduced/no programs to calculate the cost-effectiveness and estimate healthcare costs saved and thus the return on investment. Model simulations of epidemic projections over many combinations of possible resource allocations were used to identify optimal allocations for reducing new infections over the next HIV budget period.

\section{$\underline{\text { Key results }}$}

- $\quad$ The HIV/AIDS response in Indonesia has rapidly scaled up over the last 10 years with annual spending almost tripling from US\$25m in 2003 to US\$70m in 2010. A total of $\$ 363 \mathrm{~m}$ was spent on the HIV/AIDS response over this period.

- HIV/AIDS spending was split roughly between: one-third treatment, care and support; one-third prevention programs; one-third indirect costs to support programs.

- The investment in the HIV/AIDS response in Indonesia over the period 2000-2010:

- Averted approximately 130,000-240,000 HIV infections, corresponding to a 53-61\% reduction in population incidence. That is, it is estimated to have cost around US\$1,500-2,800 per infection averted overall or US\$500-800 in direct prevention funding per infection averted. The vast majority of the benefit is estimated to have occurred in non-Papua regions.

- Led to scale-up of life-sustaining antiretroviral therapy (ART), with $\sim \mathbf{2 0 , 0 0 0}$ people living with HIV maintained on ART by 2010.

- Averted an estimated 11,000-13,000 HIV/AIDS-related deaths.

- Approximately $42 \%$ of spending for prevention was allocated to most at-risk populations (MARPs): injecting drug users (IDUs); female sex workers (FSWs); waria and men who have sex with men (MSM). Most of these funds were spent on programs for IDUs (67\%) and relatively little was spent on programs for MSM (3\%), with $30 \%$ spent on programs for FSWs.

- The cost-effectiveness of the HIV prevention programs were assessed by calculating the direct cost per quality-adjusted life year gained, over the period 2000-2010. 
- Prevention programs for MSM/waria were deemed to be the most cost-effective programs at US\$61-114 per QALY gained. These programs had moderate populationlevel impact, averting an estimated 19,000-52,000 HIV infections.

- The next most cost-effective programs were needle-syringe programs (NSPs) targeting IDUs (\$US105-321 per QALY gained). The relatively large scale-up of NSPs had moderate-to-large population-level impact, averting 57,000-102,000 HIV infections.

- Resources for prevention among IDUs were allocated in roughly similar amounts between NSPs and methadone maintenance therapy (MMT). However, NSPs were estimated to be approximately twice as cost-effective in preventing new HIV infections compared to MMT. This is largely due to the substantially higher unit costs of MMT and retention-related individual-level efficacy.

- Prevention programs prioritized to FSWs were estimated to avert 4,200-9,200 HIV infections and cost US\$3,073-6,688 per QALY gained.

- Prevention programs aimed at the general population did not have evidence of noticeable impact and were deemed not to be cost-effective.

- Whether the prevention programs implemented over the period 2003-2010 are deemed to be cost-effective is questionable according to some willingness to pay thresholds, especially considering that these cost-effectiveness ratios do not include indirect costs which would inflate the estimates by $50-100 \%$. However, the programs could still be considered acceptable according to other willingness to pay thresholds.

- The economic benefits of the programs are pronounced if long-term projections are considered, as infections averted lead to aversions of clinical and disease-related outcomes in the future.

- Financial investment in HIV/AIDS programs over 2000-2010 totaling US $\$ 363 \mathrm{~m}$ is estimated to be entirely recovered in healthcare cost savings by 2050, with a total future return on investment of $\$ 1.15-\$ 1.32$ for every $\$ 1$ invested (3\% discounting). Consideration of indirect costs reduces the future return to $\sim \$ 0.55-0.63$ for every $\$ 1$ invested.

\section{$\underline{\text { Recommendations }}$}

- Relatively large management and other indirect costs reduce the overall cost-effectiveness of the HIV programs in Indonesia. With the need to do more with less funding, there is large need for greater technical efficiency and reduction of overhead costs.

- It is important to maximize allocative efficiency by distributing resources across programs in ways that minimize the expected number of new HIV infections.

- There is likely to be a shift in dominant modes of HIV transmission in Indonesia, from injecting to sexual. But to ensure IDU-driven transmission continues to be curtailed, IDU programs need to be maintained first because large increases can arise without control efforts. However, there is urgent need to ensure that emerging sexual transmission epidemics are mitigated before they become further established.

- It was estimated that a reallocation of current funding levels could result in $\sim 19 \%$ reduction in total new HIV infections if resources were allocated in an optimal way. The 
optimal allocation of prevention funding would shift funding from general prevention programs towards increased resources for MARPs: first substantially increase harm reduction for IDUs, particularly NSPs, and then base programs for MSM, followed by MMT programs to the reachable target population. It would then be most allocatively efficient to further scale-up MSM programs and commence scale-up of programs for FSW and their clients. Each program, but particularly FSW programs, should then be scaled up further as more resources become available.

- FSWs are important in acting as a bridge for HIV transmission from MARPs to the general population, particularly over longer time horizons. Therefore, with a long-term view it is important to target these groups but it is essential that programs targeting FSWs and their clients are made more efficient.

- Although non-MARP targeted programs should be reduced, sexual partners of MARPs and other discordant couples should be covered as a priority.

- With regards to the objective of minimizing new HIV infections, minimizing deaths and maximizing total health benefits (i.e. QALYs), using antiretroviral treatment for prevention (i.e., initiating therapy for people with CD4 counts greater than 350 ) should not be a large priority with currently available resources because greater benefits could be gained through behavioral prevention programs (harm reduction).

- However, scaling up ART to considerably greater levels for the large number of people who are treatment-eligible is essential for addressing the objective of reducing overall morbidity and mortality among people living with HIV and improving population health.

- Financial data were available at the national level and did not differentiate by provinces. However, with budget decreases, the government will need to re-focus the programs and prioritize selected provinces. The fiscal capacity of districts will be of importance, with the central government providing subsidy in order to address funding gaps. 


\section{Background}

Estimating the resources needed in response to HIV/AIDS epidemics is critical for determining the most efficient and effective approach to reducing new infections. HIV/AIDS effectiveness evaluation and cost-effectiveness analyses are important tools for understanding what HIV investments have bought, whether the interventions averted new infections and AIDS deaths, and at what cost. They can support decision-making and policy development by informing the HIV/AIDS response with its overall goals of minimizing the burden of disease and maximizing health outcomes.

The majority of financial resources to support the HIV/AIDS response in Indonesia have been donated by the Global Fund to Fight AIDS, Tuberculosis and Malaria (GFATM). Multilateral organizations, such as the GFATM, and other international donors have decreased their commitment to invest in HIV/AIDS in Indonesia as well as in many other middle-income countries. Indonesia will not be applying for GFATM support at the end of the current round in 2014. The Indonesian government, which currently contributes $~ 30 \%$ to the overall HIV budget, intends to increase its contribution to fill in as much of the funding gap as possible, and as efficiently as possible, over the next national strategy timeframe 2015-2019.

The goal of this study is to estimate the cost-effectiveness and return on investment of HIV programs implemented over the period 2000-2010 and to contribute to the improvement of the effectiveness and efficiency of HIV prevention responses in Indonesia, specifically to inform the prioritization of resources for the 2015-2019 national HIV budget in the context of reductions in international donor funding. This study aims to establish evidence of cost-effectiveness and optimal allocations of limited resources for greatest epidemiological impact. It does this through:

1. collating all available data on the funding of HIV prevention programs, from National AIDS Spending Assessments and other program unit cost calculations, in the non-Papua and Papua regions of Indonesia and the breakdown of Indonesia's national budget;

2. assessing relationships in data between funding for programs, risk behaviors and the resulting HIV epidemics in Indonesia;

3. estimating the cost-effectiveness of past HIV prevention programs in Indonesia, with the use of data-driven modeling to estimate the number of HIV infections and deaths averted and QALYs gained due to HIV programs in comparison to the costs of the programs;

4. projecting the impact of reductions in the 2015-2019 HIV budget on the HIV epidemic and determining the optimal allocation of available funding to reduce the adverse impact of these reductions.

This study also estimates the optimal balance of funding between prevention programs and treatment, considering the use of antiretroviral therapy for prevention and improved survival and health of the population of people living with HIV. 


\section{Methods}

\section{Mathematical epidemic transmission model}

A mathematical epidemic model was developed to investigate the impact of HIV prevention programs in Indonesia in the non-Papua and Papuan regions. This model is a modified version of the HIV in Indonesia Model (HIM), specifically designed for Indonesia using best-practice HIV epidemic modeling techniques and incorporating realistic biological transmission processes, detailed infection progression, and sexual mixing patterns and drug injection behaviors. Informed by available HIV surveillance data, the model includes 10 distinct population groups: Male and female injecting drug users (IDU), Direct and indirect female sex workers (FSWs), Clients of FSWs, Waria, Bisexual men, Men who have sex with men (MSM) and low-risk males and females in the general population. This model is similar in approach to the Asian Epidemic Model (AEM) with Spectrum, which has previously been applied to Indonesia, but the current model is flexible to Indonesian-specific characteristics and data and is amenable to analyses directly relevant to the current research questions including full health economic analyses, production of uncertainty bounds, and resource optimization. Further details of HIM are available in Appendix 2.

\section{Model calibration}

Most of the model inputs associated with sexual and injecting risk behaviors were taken from BSS or IBBS data from previous studies in Indonesia, endorsed by the Ministry of Health. Figure 1 shows cumulative new HIV infections in whole of Indonesia from 2000-2010. (See Appendix 3: Calibration of model to the HIV epidemics in non-Papua and Papua.)

Figure 1: Cumulative number of new infections from 2000 to 2010 (National), by population group

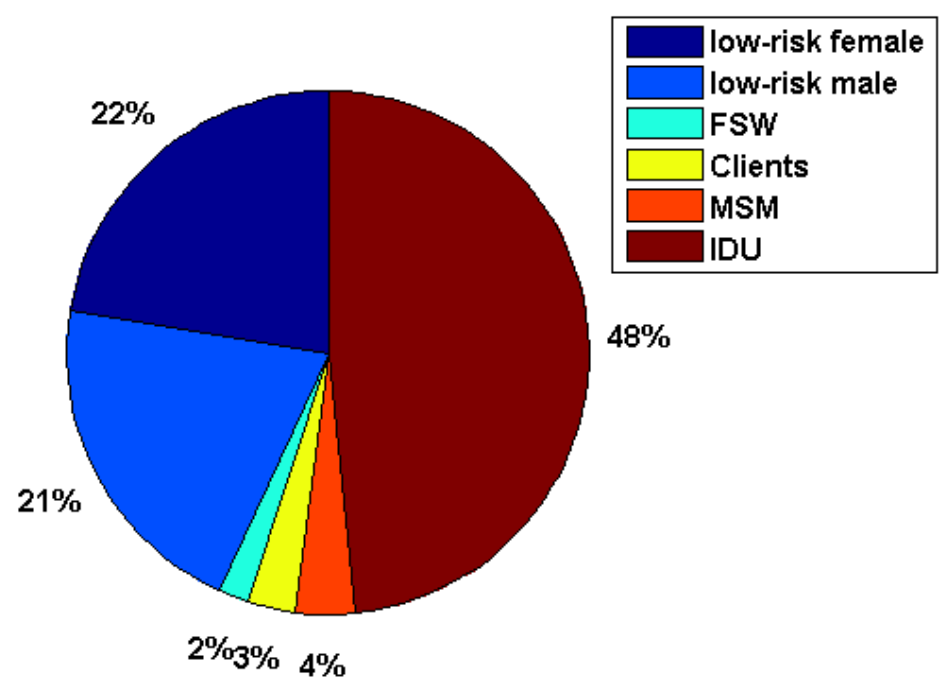




\section{Collation of costing data}

\section{Costs of HIV/AIDS prevention programs in Indonesia}

Using available HIV funding, spending and budgeting data from Indonesia - informed by the National AIDS Spending Assessment (NASA) and supported by other available sources including an Indonesian costing for harm reduction study (by the University of Indonesia for the World Bank) and other program unit cost calculations - the amount of funding allocated to each prevention program (targeted to specific priority populations or the general population) over 2003-2010 was calculated. Itemized breakdowns of total budgets to program areas and supporting costs were only available for years 2008-2010. NASA exercises were conducted for 2008, 2009 and 2010. Due to differences in cost disaggregation in 2008 compared to 20092010 associated with different approaches taken, the more consistent spending breakdown for 2009-2010 was used to calculate proportional allocations to be applied to prior years. The total spending on all programs in each previous year was multiplied by the average proportion allocation to each program in 2009-2010 to estimate the disaggregated program-specific cost by year. Five core prevention programs were identified:

1. Programs for FSW and their clients;

2. Programs for MSM;

3. Needle-syringe programs (NSP);

4. Methadone Maintenance Therapy; and

5. $\quad$ Programs for Low-risk population (general population).

HIV spending data (that were sufficiently broken down by item) were only available at the national level. However, given the distinct HIV epidemics in Papua compared to non-Papua regions, it was important that assessments of epidemiological impacts were split across these broad regions. Assumptions were made regarding geographical cost and program breakdowns (see Appendices).

In order to split spending into different programs for IDUs, groupings used in the Costing for Harm Reduction 2007 study were adopted. The final budget breakdown was determined through a systematic process outlined in Appendix 1 and a summary of its outcomes is presented in Table 1.

\section{Costs for economic analyses and return on investment}

Health care costs as well as programmatic costs were required for the cost-effectiveness analyses. Unit healthcare costs were agreed upon among investigators at UNSW and the University of Indonesia, School of Public Health (see Table 9 in Appendix 1).

Consumer price indices for healthcare as well as discount rates were incorporated into the monetary values to calculate the total investment in each program for the period 2000-2010 and 2000-lifetime, where lifetime costs are computed in the period 2000-2090 with discounting at $0 \%, 3 \%$ and $10 \%$.

\section{Economic analysis methods}

Using HIM, the effectiveness of past HIV programs were calculated by comparing the expected number of new infections and HIV/AIDS related deaths according to actual conditions with the 
estimated numbers under counterfactual scenarios of reduced-to-no funding for specific programs. Data were used to formulate evidence-based assumptions on the relationship between funding and risk factors, particularly for hypothetical intermediate funding levels.

An example of counterfactual scenarios and the resulting projected change in the HIV epidemic is shown in Figure 1. Two choices of counterfactual scenarios are used for sensitivity analysis: an extreme scenario of no funding with baseline risk factors remaining constant over time and a second scenario whereby the midpoint between the actual change in behaviors and the no funding scenario is simulated over time to account for the potential indirect effects of other programs and exogenous factors. Further details of all counterfactual assumptions and the costeffectiveness calculations are provided in Appendix 5. HIM was used to project epidemic trajectories according to the counterfactual scenarios; comparing these trajectories with the calibrated epidemic trajectory according to actual conditions resulted in an estimation of the effectiveness of the programs.

Figure 2: Example of assumed counterfactuals (red) compared with actual conditions (blue) for evaluating the effectiveness of HIV prevention programs for MSM in non-Papua. The solid red curve represents no funding with baseline risk factors remaining constant over time and the dashed red curve is the midpoint between the actual change in behaviors and the no funding scenario which accounts for potential indirect effects of other programs.
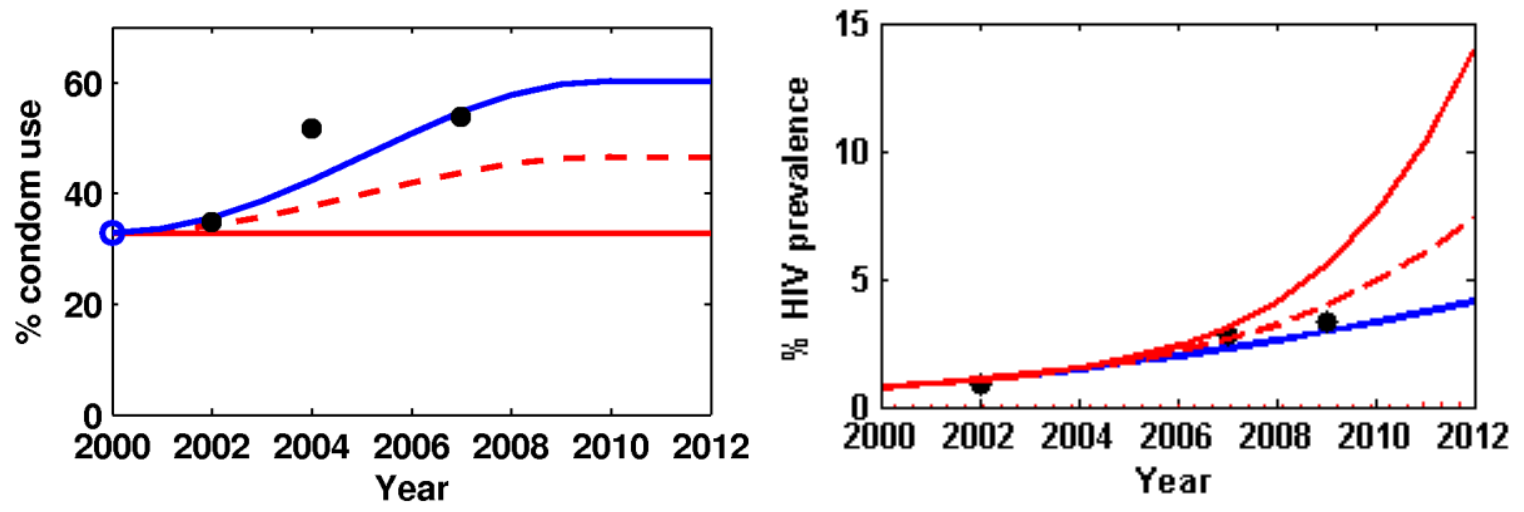

The cost-effectiveness of each program was then assessed by comparing the spending of each program, as well as estimated annual healthcare costs incurred / saved, with the estimated effectiveness of the programs. An estimate of the incremental cost-effectiveness ratio (ICER) of each program was calculated based on the counterfactual scenarios. Two time horizons were used: 2000-2010 and 2000-lifetime. This process was carried out for each intervention program targeting specific population groups. Further details are in Appendix 5. This economic analysis was conducted using detailed data on costs. The outcome of interest from the economic analysis was Quality Adjusted Life Years (QALYs). Discounting and consumer price indices $(\mathrm{CPI})$ were used to assess the value of money at different time periods. Healthcare CPIs were based on year-to-year data from Global Rates CPI, which sources its data from Statistics Indonesia. 
The timeline from 2000-2010 was investigated to estimate the number of HIV infections averted due to implementation of the prevention programs in the past. The model was then used to project long-term health outcomes and healthcare costs incurred over the next 80 years (20112090) in order to estimate the future benefits of the past programs. The cost-effectiveness of the HIV prevention programs were assessed by calculating the cost per quality-adjusted life year gained, over the period 2000-2010. A return on investment analysis considered the future healthcare costs saved that are attributable to the past financial investment in HIV/AIDS programs.

\section{Optimal allocation of funding for reduced HIV prevention budget}

In order to calculate an optimal allocation of funding across HIV programs, it was necessary to make assumptions about the relationship between a given program funding amount and targeted risk behaviors of the program. The approach taken in this study is illustrated in Figure 3 through an example - of Papuan indirect sex workers.

Figure 3: Example of the relationship between spending on FSW/client programs and the HIV epidemic.

\section{Relationship between funding level and indirect FSW behaviour in Papua}
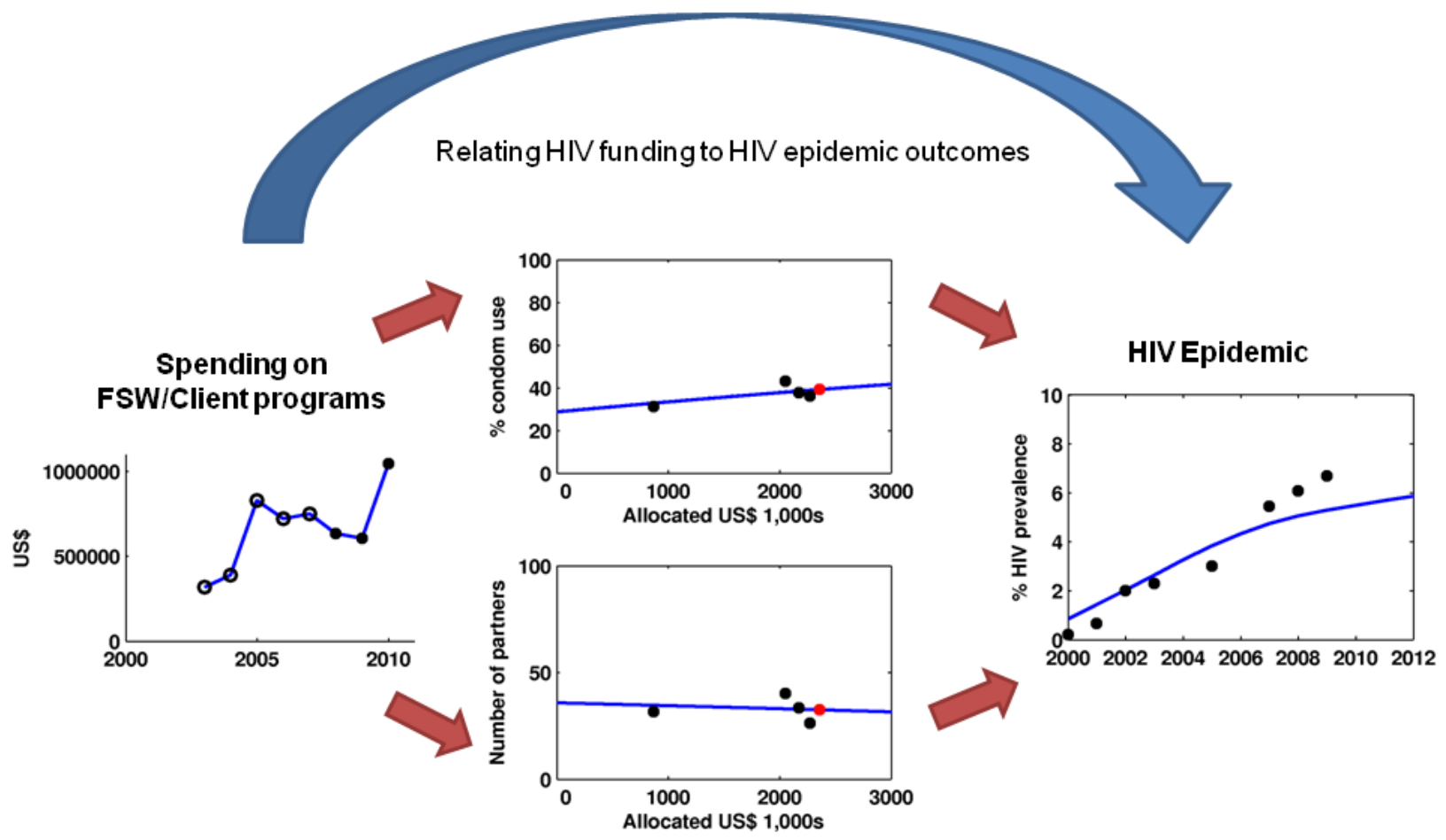
In summary, the analysis was done by the following procedure:

1. Identify indicators / risk factors associated with each prevention program (e.g. condom use and number of clients for FSW programs).

2. Assess trends over time in spending on each program and the identified risk factors - link these variables together in a data association between risk factor and level of program spending.

3. Fit sigmoid/logistic curves to the data associations between risk factor and program spending. These sigmoid relationships describe predicted values for indicators under different potential spending levels.

4. For a given total budget available, a very large number of possible combinations of funding breakdowns across programs was sampled and the sigmoid relationships were used to assume a given program response on the targeted risk factors. HIM was then used to simulate the epidemic trajectories according to these conditions and estimate the expected number of new HIV infections.

\section{Identifying optimal funding allocations}

HIM was used to project the number of HIV infections and HIV/AIDS related deaths over 20102019 according to different funding allocations to HIV/AIDS prevention, treatment and supporting programs. Funding levels are kept similar from 2010 to 2014 according to allocation data from 2009-2010. The expected change in funding (decrease) is assumed to occur in 2015, hence different allocation of funding from 2015 to 2019 is analyzed. For a given total budget available, 4,000 distributions of funding breakdowns across all programs were determined through computational sampling across the range of all possible distributions. Two sets of simulations were run: (1) maintain current rates of ART; (2) increased ART programs, accounting for effects of treatment as prevention.

The expected behavioral and clinical outcomes associated with each funding scenario were identified (through the pre-determined, data-driven sigmoid/logistic relationships) for each funding allocation and HIM was used to simulate the expected epidemic trajectory according to these conditions. The funding allocation to each program that resulted in the minimum cumulative number of new HIV infections was identified as the optimal allocation of available funding for HIV prevention programs. This is illustrated in Error! Reference source not found.. he optimal allocation methodology is presented in more detail in Appendix 6. Using this methodology, the optimal allocation of increased domestic funding and reductions in inefficiencies was also identified. The optimal allocation of resources for preventing new infections was investigated with core prevention methods (harm reduction), along with introducing programs based on using antiretroviral treatment as prevention in combination with other programs. 
Figure 4: Example of optimizing the allocation of resources in the 2015 to 2019 HIV budget to minimize new HIV infections

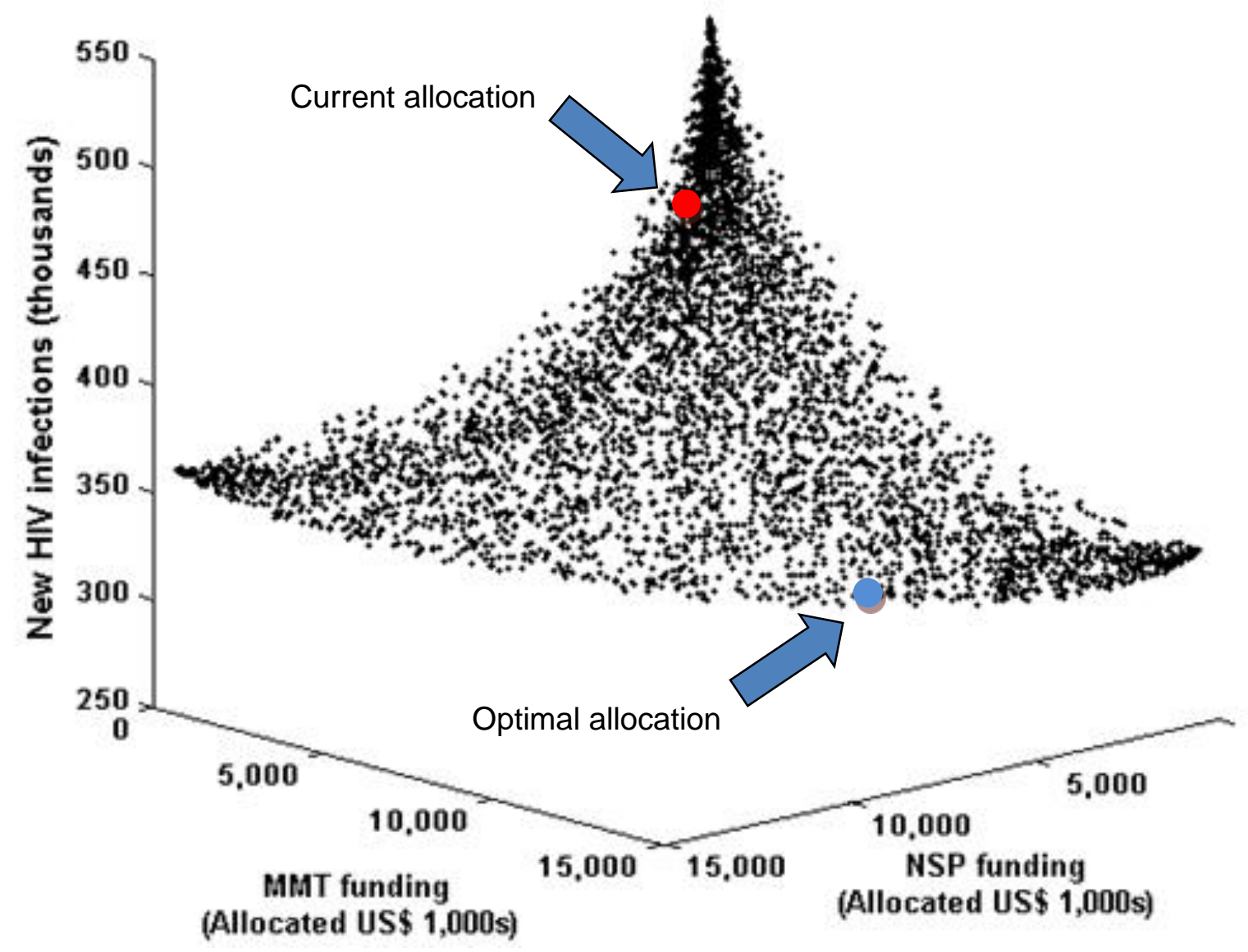

\section{Key Assumptions and Data Gaps}

Below is a list of key assumptions made in carrying out the analyses. These assumptions need to be considered carefully when interpreting the study findings.

\section{Costing and spending breakdown}

- Total HIV funding for each year was assumed to have the same proportional breakdown as the 2009-2010 NASA reports.

- Spending with an unknown allocation as well as funding for the STI, PMTCT, PEP and PLWH NASA categories was reallocated to funding for MARPs.

- Funds spent on vulnerable and accessible populations (VAP), youth out of school (YOS), youth in school (YIS) and drug-dependent therapy (DDT) were reallocated to the general population. 
- Funds spent on health services unrelated to risk behavior (such as universal prevention in health services) were allocated into indirect costs associated with prevention programs.

- Drug-dependent therapy (DDT) was assumed to have no impact on IDU behavior. Funding for DDT was reallocated to prevention spending aimed at the general population.

- $\quad$ BCC, VCT and NSP were assumed to have an impact on IDU condom use, IDU partner numbers, IDU testing, and syringe sharing.

- MMT was assumed to have an impact on the percentage of IDUs on MMT and the average number of injections of IDUs. Numbers of injections for IDUs were assumed to account for those on MMT.

- Indirect costs were assumed to include the following NASA categories: Program management and administrative strengthening, Human resources, Social protection and social services, Enabling environment and Research.

- Spending on sex workers and their clients were assumed to affect only female sex workers, which therefore excludes male sex workers and waria sex workers. Behavior of waria, which includes sex with casual and commercial partners, is assumed to potentially be impacted by programs for MSM. Male sex workers are not included in the model.

Relationship between spending and risk behavior - sigmoid/logistic curve fits to data

- A sigmoid/logistic relationship between spending on a program and changes in behavior was assumed, fitted by empirical data for the relevant indicators.

- Based on available data and what has occurred in other countries, it was assumed that a maximum (or minimum depending on parameter) saturation value for each behavioral parameter existed as spending increases.

- The parameter value when there is no funding for a prevention program is assumed to equal the calibrated value for the year 2000 from the model.

- Non-targeted prevention programs were assumed to affect condom use, partner numbers and testing rates in the low-risk/general populations.

- Prevention programs for sex workers and their clients were assumed to affect condom use, partner numbers, number of clients, and testing rates for FSWs and their clients

- Prevention programs for MSM/waria were assumed to affect condom use, partner numbers and testing rates.

- It was assumed that NSPs affect the percentage of IDUs that share syringes and the percentage of injections that are shared.

- It was assumed that MMT affects the percentage of IDUs taking methadone and the average number of injections per year. 
$\underline{\text { Model }}$

- Once categorized, it was assumed that over the time period of analysis, individuals do not move between population groups. For example, FSWs do not stop being sex workers, IDUs always remain IDUs etc.

- Diagnosed individuals are assumed to have the same characteristics as those undiagnosed except they can begin ART.

- Individuals who fail treatment are assumed to no longer be on ART.

$\underline{\text { Counterfactual scenarios and cost-effectiveness calculations }}$

- It was assumed that all programs began in the year 2000.

- The change in parameter values over time is obtained from the calibrated model, informed by the data-driven relationships between spending and the relevant indicator associated with the parameter.

- Counterfactual scenarios represent what is assumed to have occurred in the absence of HIV/AIDS programs. It was assumed that removing spending for a particular program meant that the model parameters affected by that program remained fixed at their year 2000 value.

- Programmatic costs are assumed to include all consumables and services for target groups.

- Health utilities were based on a meta-analysis of utility estimates for HIV/AIDS in adults (Tengs TO, Lin TH. Medical Decision Making 2002: 22:475-481).

Optimal allocation of spending analysis

- A constant amount was allocated to essential funding, consisting of OVC, ART and associated indirect costs.

- It was assumed that no programs had an effect on the population size of MARPs.

- Program spending was assumed to be directly related to the program coverage in MARPs.

- A fixed 47.5:52.5 ratio was assumed between prevention and indirect costs. Only direct costs were used in program budget assessments. Indirect costs would therefore inflate the direct costs by approximately double.

- The assumed budget in 2011-2014 is equal to the budget allocation in 2010. Decreases in budget were assumed to occur in 2015 and maintained at the same annual value during 2015-2019.

\section{$\underline{\text { Treatment as prevention scenarios }}$}

- Treatment as prevention scenarios were investigated by determining the number of people that can begin ART for a fixed amount of funding. The testing and treatment initiation rates were then adjusted in HIM to match this number by assuming:

- The same treatment initiation rate for each group of people, categorized by CD4 count;

- A doubling of the testing rate. 


\section{Data gaps}

- There are insufficient data on the behavior of the low risk population to accurately assess the impact of general population HIV programs. For sensitivity analysis, a $5-10 \%$ decrease in condom use among low-risk populations was assumed in the absence of funding for general population.

- Spending data are not broken down to the regional level so the impact of programs in each region cannot be assessed.

- Yearly costs were estimated from annual spending as reported in NASA reports. These yearly costs are reported as an aggregate cost that included both international spending and domestic spending. Local government spending in each year was collected from provinces and reported as aggregate amounts nationally. However, it must be noted that provinces included in the aggregate cost differed in each year. Specifically, older data would have included fewer provinces and hence data quality is expected to be poorer.

- A large part of the NASA is unclassified and an accurate breakdown of the HIV budget cannot be achieved.

- Funding for IDU harm reduction programs is not broken down into MMT, NSP and DDT making it hard to assess the cost-effectiveness of these programs; assumptions on allocation had to be made (see Tables and Appendices).

- There are limited data on HIV diagnostic testing.

- Due to little published information on the healthcare costs for individuals in different health stages of HIV/AIDS, these were assumed to be the same across different CD4 categories. 


\section{Results}

The HIV/AIDS response in Indonesia has rapidly scaled up over the last 10 years with annual spending almost tripling from $\sim 25 \mathrm{~m}$ in 2003 to $\sim$ US $\$ 70 \mathrm{~m}$ in 2010 . A total of $\$ 363 \mathrm{~m}$ was spent on the HIV/AIDS response over this period. HIV/AIDS spending was split roughly between: onethird treatment, care and support; one-third prevention programs; one-third indirect costs to support programs.

Total annual direct costs for prevention increased from $\$ 7 \mathrm{~m}$ in 2003 to $\$ 18 \mathrm{~m}$ in 2010. In 2010, approximately $43 \%$ of annual spending for prevention were allocating to direct costs for programs aimed at target populations (57\% to indirect costs, which include universal precautions in health care systems). Twenty-two percent of these direct costs were allocated to low-risk populations while 78\% were allocated to most at-risk populations (MARPs): injecting drug users (IDUs); female sex workers (FSWs); men who have sex with men (MSM) and waria. Total spending in the past decade in HIV prevention programs of $\$ 101 \mathrm{~m}$ was distributed to populations as follows: $\$ 59 \mathrm{~m}$ to low-risk, $\$ 14.6 \mathrm{~m}$ to NSP, $\$ 13.7 \mathrm{~m}$ to MMT, $\$ 1.3 \mathrm{~m}$ to MSM and $\$ 12.7$ to FSW and their clients (see Table 1). Expenditures for care and treatment totaled $\$ 127 \mathrm{~m}$, with $\$ 72 \mathrm{~m}$ spent on ART alone.

\section{Cost-effectiveness of Past HIV Prevention Programs in Indonesia}

The epidemiological benefits and economic analyses of each HIV program over 2000-2010 are summarized in Table 2. Overall, the HIV response in Indonesia averted $\sim 130,000-240,000$ new infections, with the vast majority in Non-Papuan regions. An estimated 11,000-13,000 total HIVrelated deaths were averted due to the HIV programs. Increases in prevalence within risk groups were also prevented, most notably HIV prevalence among IDUs could have been substantially greater than the already high levels.

Programs for MSM and waria were most cost-effective at \$61-114 per QALY gained, followed by NSPs at \$105-321 per QALY gained. The economic benefits of HIV programs are further pronounced if long-term projections are considered. Across all HIV prevention programs, considering future benefits, there was a return on investment of $\$ 1.15-1.32$ for every $\$ 1$ invested in direct costs. Full cost recovery on direct is expected by year 2050 . However, indirect costs account for a considerable amount of expenditure, such that the overall future return on investment is estimated to be $\$ 0.55-0.63$ for every $\$ 1$ in the prevention and management budget. 
Table 1: Spending by year and program area

\begin{tabular}{|c|c|c|c|c|c|c|c|c|c|c|}
\hline \multirow{2}{*}{\multicolumn{2}{|c|}{ Spending item }} & \multicolumn{8}{|c|}{ Amount spent in US\$ } & \multirow{2}{*}{$\begin{array}{c}\text { Total } \\
\text { spending, } \\
\text { adjusted for } \\
\text { CPI } \\
\end{array}$} \\
\hline & & 2003 & 2004 & 2005 & 2006 & 2007 & 2008 & 2009 & 2010 & \\
\hline \multicolumn{2}{|c|}{ HIV spending TOTAL overall } & $\$ 24,890,908$ & $\$ 30,407,332$ & $\$ 64,885,625$ & $\$ 56,576,587$ & $\$ 58,671,397$ & $\$ 49,563,286$ & $\$ 60,285,420$ & $\$ 69,146,880$ & $\$ 362,995,804$ \\
\hline GP/VAP/YOS/YIS* & $\begin{array}{l}\text { General } \\
\text { population }\end{array}$ & $\$ 4,023,009$ & $\$ 4,914,604$ & $\$ 10,487,180$ & $\$ 9,144,226$ & $\$ 9,482,801$ & $\$ 8,010,697$ & $\$ 10,495,206$ & $\$ 10,424,371$ & $\$ 58,651,477$ \\
\hline NSP & $\begin{array}{l}\text { Needle and } \\
\text { syringe } \\
\text { programs }\end{array}$ & $\$ 1,008,105$ & $\$ 1,231,525$ & $\$ 2,627,928$ & $\$ 2,291,405$ & $\$ 2,376,246$ & $\$ 2,007,359$ & $\$ 2,608,627$ & $\$ 2,633,502$ & $\$ 14,697,681$ \\
\hline MMT & $\begin{array}{l}\text { Methadone } \\
\text { maintenance } \\
\text { therapy }\end{array}$ & $\$ 939,584$ & $\$ 1,147,818$ & $\$ 2,449,308$ & $\$ 2,135,658$ & $\$ 2,214,733$ & $\$ 1,870,919$ & $\$ 2,431,318$ & $\$ 2,454,503$ & $\$ 13,698,678$ \\
\hline MSM/Waria & $\begin{array}{l}\text { Programs } \\
\text { targeting } \\
\text { MSM (and }\end{array}$ & $\$ 91,310$ & $\$ 111,547$ & $\$ 238,028$ & $\$ 207,547$ & $\$ 215,231$ & $\$ 181,819$ & $\$ 187,356$ & $\$ 287,456$ & $\$ 1,332,428$ \\
\hline FSW/Clients & $\begin{array}{l}\text { Programs } \\
\text { targeting sex } \\
\text { workers and } \\
\text { their clients }\end{array}$ & $\$ 871,365$ & $\$ 1,064,480$ & $\$ 2,271,474$ & $\$ 1,980,597$ & $\$ 2,053,931$ & $\$ 1,735,080$ & $\$ 2,171,385$ & $\$ 2,359,698$ & $\$ 12,706,069$ \\
\hline IC & $\begin{array}{l}\text { Indirect } \\
\text { costs }\end{array}$ & $\$ 9,224,079$ & $\$ 11,268,357$ & $\$ 24,045,333$ & $\$ 20,966,167$ & $\$ 21,742,462$ & $\$ 18,367,176$ & $\$ 21,226,693$ & $\$ 26,738,364$ & $\$ 134,545,686$ \\
\hline ART & $\begin{array}{l}\text { Antiretroviral } \\
\text { treatment }\end{array}$ & $\$ 4,956,626$ & $\$ 6,055,134$ & $\$ 12,920,934$ & $\$ 11,266,322$ & $\$ 11,683,470$ & $\$ 9,869,735$ & $\$ 11,280,191$ & $\$ 14,494,180$ & $\$ 72,302,112$ \\
\hline Other CST & $\begin{array}{l}\text { Other care, } \\
\text { support and } \\
\text { treatment }\end{array}$ & $\$ 3,736,268$ & $\$ 4,564,315$ & $\$ 9,739,705$ & $\$ 8,492,470$ & $\$ 8,806,913$ & $\$ 7,439,734$ & $\$ 9,802,383$ & $\$ 9,626,148$ & $\$ 54,469,766$ \\
\hline ovc & $\begin{array}{l}\text { Orphans } \\
\text { and } \\
\text { vulnerable } \\
\text { children }\end{array}$ & $\$ 40,561$ & $\$ 49,551$ & $\$ 105,736$ & $\$ 92,196$ & $\$ 95,609$ & $\$ 80,767$ & $\$ 82,261$ & $\$ 128,658$ & $\$ 591,909$ \\
\hline $\begin{array}{l}\text { *Low-risk males an } \\
\text { ** Assumed as indir } \\
{ }^{\star \star \star} \text { Assumed as ess } \\
{ }^{* \star \star \star} \text { Assumed as }\end{array}$ & $\begin{array}{l}\text { females (GP), vu } \\
\text { ct costs to imple } \\
\text { ntial costs }\end{array}$ & $\begin{array}{l}\text { nerable and acce } \\
\text { enting preventior }\end{array}$ & $\begin{array}{l}\text { sible populations } \\
\text { programs; incluc }\end{array}$ & $\begin{array}{l}\text { (VAP), youth-in- } \\
\text { es prevention prc }\end{array}$ & $\begin{array}{l}\text { chool (YIS), yout } \\
\text { grams within hea }\end{array}$ & $\begin{array}{l}\text { 1-out-of-school (' } \\
\text { th care facilities }\end{array}$ & $\begin{array}{l}\text { OS) } \\
\text { universal prec }\end{array}$ & ns, blood safe & & \\
\hline
\end{tabular}


Table 2: Estimated epidemiological impact and cost-effectiveness of past HIV/AIDS programs in Indonesia. Ranges refer to results from two counterfactual assumptions: (i) no funding with baseline risk factors remaining constant over time and (ii) midpoint between the actual change in behaviors and the no funding scenario ${ }^{9}$.

\begin{tabular}{|c|c|c|c|c|c|c|c|}
\hline Outcome & Current conditions & $\begin{array}{c}\text { Without FSW/Clients } \\
\text { program }\end{array}$ & Without MSM & Without MMT & Without NSP & $\begin{array}{c}\text { No programs aimed } \\
\text { at low-risk }\end{array}$ & $\begin{array}{c}\text { No HIV/AIDS core } \\
\text { prevention programs } \\
(3)\end{array}$ \\
\hline \multicolumn{8}{|c|}{ Epidemiological indicators } \\
\hline \multicolumn{8}{|c|}{ PAPUA } \\
\hline $\begin{array}{c}\text { Overall Prevalence } \\
(2010)\end{array}$ & $0.19 \%$ & $0.20 \%-0.21 \%$ & - & - & - & $0.19 \%-0.19 \%$ & $0.20 \%-0.21 \%$ \\
\hline \multicolumn{8}{|c|}{ Prevalence among risk groups (2010) } \\
\hline low-risk males & $0.22 \%$ & $0.22 \%-0.22 \%$ & - & - & - & $0.22 \%-0.22 \%$ & $0.22 \%-0.22 \%$ \\
\hline low-risk females & $0.17 \%$ & $0.18 \%-0.19 \%$ & - & - & - & $0.17 \%-0.17 \%$ & $0.18 \%-0.19 \%$ \\
\hline DFSW & $14.07 \%$ & $16.15 \%-18.83 \%$ & - & - & - & $14.07 \%-14.07 \%$ & $16.16 \%-18.83 \%$ \\
\hline IDFSW & $6.27 \%$ & $7.72 \%-9.63 \%$ & - & - & - & $6.27 \%-6.27 \%$ & $7.73 \%-9.63 \%$ \\
\hline Clients of FSW & $0.62 \%$ & $0.73 \%-0.88 \%$ & - & - & - & $0.62 \%-0.62 \%$ & $0.73 \%-0.88 \%$ \\
\hline $\begin{array}{c}\text { Cumulative new } \\
\text { infections }\end{array}$ & $26,453.90$ & $28,100-30,259$ & - & - & - & $26,476-26,567$ & $28,217-30,283$ \\
\hline $\begin{array}{l}\text { Cumulative HIV- } \\
\text { related deaths }\end{array}$ & $2,803.60$ & $2,854-2,914$ & - & - & - & $2,805-2,810$ & $2,860-2,915$ \\
\hline Infections averted & & $1,646-3,805$ & - & - & - & $23-113$ & $1,763-3,829$ \\
\hline Deaths averted & & $50-110$ & - & - & - & $41,061.00$ & $56-111$ \\
\hline $\begin{array}{c}\text { Cumulative } \\
\text { number of people } \\
\text { starting 1st line } \\
\text { ART } \\
\end{array}$ & $1,460.40$ & $1,455-1,459$ & - & - & - & $1,461-1,462$ & $1,455-1,461$ \\
\hline $\begin{array}{c}\text { Cumulative } \\
\text { number of people } \\
\text { starting } 2 \text { nd line } \\
\text { ART } \\
\end{array}$ & 3.24 & $3-3$ & - & - & - & $3-3$ & $3-3$ \\
\hline \multicolumn{8}{|c|}{ NON-PAPUA } \\
\hline \multicolumn{8}{|c|}{ Prevalence on 2010} \\
\hline
\end{tabular}




\begin{tabular}{|c|c|c|c|c|c|c|c|}
\hline Overall Prevalence & $0.19 \%$ & $0.20 \%-0.20 \%$ & $0.20 \%-0.21 \%$ & $0.21 \%-0.23 \%$ & $0.22 \%-0.25 \%$ & $0.19 \%-0.19 \%$ & $0.22 \%-0.25 \%$ \\
\hline \multicolumn{8}{|c|}{ Prevalence among risk groups (2010) } \\
\hline low-risk males & $0.04 \%$ & $0.04 \%-0.04 \%$ & $0.04 \%-0.04 \%$ & $0.04 \%-0.04 \%$ & $0.04 \%-0.04 \%$ & $0.04 \%-0.04 \%$ & $0.04 \%-0.04 \%$ \\
\hline low-risk females & $0.02 \%$ & $0.02 \%-0.02 \%$ & $0.02 \%-0.02 \%$ & $0.02 \%-0.02 \%$ & $0.02 \%-0.02 \%$ & $0.02 \%-0.02 \%$ & $0.02 \%-0.02 \%$ \\
\hline DFSW & $5.95 \%$ & $6.67 \%-7.45 \%$ & $5.95 \%-5.95 \%$ & $6.24 \%-6.54 \%$ & $6.43 \%-6.89 \%$ & $5.95 \%-5.95 \%$ & $7.35 \%-9.36 \%$ \\
\hline IDFSW & $3.02 \%$ & $3.38 \%-3.80 \%$ & $3.02 \%-3.02 \%$ & $3.15 \%-3.28 \%$ & $3.23 \%-3.44 \%$ & $3.02 \%-3.02 \%$ & $3.59 \%-4.59 \%$ \\
\hline Clients of FSW & $0.34 \%$ & $0.38 \%-0.43 \%$ & $0.34 \%-0.34 \%$ & $0.34 \%-0.35 \%$ & $0.34 \%-0.35 \%$ & $0.34 \%-0.34 \%$ & $0.37 \%-0.44 \%$ \\
\hline MSM & $3.30 \%$ & $3.30 \%-3.30 \%$ & $4.95 \%-7.66 \%$ & $3.30 \%-3.30 \%$ & $3.30 \%-3.30 \%$ & $3.30 \%-3.30 \%$ & $4.95 \%-7.67 \%$ \\
\hline Waria & $10.75 \%$ & $10.75 \%-10.75 \%$ & $15.84 \%-24.35 \%$ & $10.75 \%-10.75 \%$ & $10.75 \%-10.75 \%$ & $10.75 \%-10.75 \%$ & $15.8 \%-24.37 \%$ \\
\hline Male IDU & $57.09 \%$ & $57.11 \%-57.13 \%$ & $57.09 \%-57.09 \%$ & $63.76 \%-70.51 \%$ & $68.78 \%-79.44 \%$ & $57.09 \%-57.09 \%$ & $76.70 \%-92.31 \%$ \\
\hline Female IDU & $57.02 \%$ & $57.02 \%-57.02 \%$ & $57.02 \%-57.02 \%$ & $63.70 \%-70.46 \%$ & $68.72 \%-79.41 \%$ & $57.02 \%-57.02 \%$ & $76.66 \%-92.29 \%$ \\
\hline $\begin{array}{c}\text { Cumulative new } \\
\text { infections }\end{array}$ & 255,587 & $259,844-264,806$ & $274,212-307,674.9$ & $286,858-316,821$ & $312,556-357,945$ & $255,647-255,707$ & $380,300-489,199$ \\
\hline $\begin{array}{c}\text { Cumulative HIV- } \\
\text { related deaths }\end{array}$ & 117,624 & $117,838-118,076$ & $118,319-119,338.5$ & $121,137-124747$ & $123,459-129,151$ & $117,630-117,635$ & $128,377-130,189$ \\
\hline Infections averted & & $4,257-9,219$ & $18,625-52,088$ & $31,271-61,234$ & $56,969-102,357$ & $60-120$ & $124,713-233,612$ \\
\hline Deaths averted & & $214-453$ & $696-1,714.7$ & $3,513-7,123$ & $5,835-11,528$ & $6-11$ & $10,753-12,565$ \\
\hline $\begin{array}{c}\text { Cumulative } \\
\text { number of people } \\
\text { starting } 1 \text { st line } \\
\text { ART } \\
\end{array}$ & 29927 & $29,874-29,779$ & $29,913-29,882$ & $30,383-30,864$ & $29,043-27,166$ & $29,927-29,927$ & $28,924-30,706$ \\
\hline $\begin{array}{c}\text { Cumulative } \\
\text { number of people } \\
\text { starting 2nd line } \\
\text { ART }\end{array}$ & 29 & $29-29$ & $29-29$ & $29-29$ & $28-29$ & $29-29$ & $28-29$ \\
\hline \multicolumn{8}{|c|}{ Cost-effectiveness } \\
\hline Total program cost & & $\$ 12,706,069.40$ & $\$ 1,332,427.60$ & $\$ 13,698,677.90$ & $\$ 14,697,680.50$ & $\$ 58,651,476.70$ & $\$ 101,086,332.00$ \\
\hline \multicolumn{8}{|c|}{ 2000-2010 } \\
\hline $\begin{array}{l}\text { QALYs gained } \\
\text { (undiscounted) }\end{array}$ & & $1,766-3,749$ & $4,364-10,626$ & $16,000-32,534$ & $26,141-52,004$ & $42-84$ & $50,144-109,522$ \\
\hline $\begin{array}{l}\text { QALYs gained } \\
\text { (discounted 3\%) }\end{array}$ & & $1,857-3,938$ & $4,566-11,074$ & $17,059-34,695$ & $27,828-55,406$ & $47-94$ & $52,820-115,958$ \\
\hline
\end{tabular}




\begin{tabular}{|c|c|c|c|c|c|c|c|}
\hline \multirow[b]{2}{*}{$\begin{array}{c}\text { Total healthcare } \\
\text { costs (undiscounted) }\end{array}$} & \multirow[b]{2}{*}{$\$ 119,615,350$} & \multirow[b]{2}{*}{$\begin{array}{c}\$ 119,888,922- \\
\$ 120,186,530\end{array}$} & \multirow[b]{2}{*}{$\begin{array}{c}\$ 120,389,286- \\
\$ 121,536,707 \\
\end{array}$} & \multirow[b]{2}{*}{$\begin{array}{c}\$ 124,220,115- \\
\$ 128,122,767\end{array}$} & \multirow[b]{2}{*}{$\begin{array}{c}\$ 125,719,845- \\
\$ 130,477,076 \\
\end{array}$} & & \\
\hline & & & & & & $\begin{array}{c}\$ 119,622,022- \\
\$ 119,628,684 \\
\end{array}$ & $\begin{array}{c}\$ 130,389,391- \\
\$ 141,652,111 \\
\end{array}$ \\
\hline $\begin{array}{l}\text { Total healthcare } \\
\begin{array}{c}\text { costs } \\
\text { (discounted } \\
3 \% \text { ) }\end{array} \\
\end{array}$ & $\$ 146,093,101$ & $\begin{array}{c}\$ 146,382,627- \\
\$ 146,697,275 \\
\end{array}$ & $\begin{array}{c}\$ 146,905,270- \\
\$ 148,100,377\end{array}$ & $\begin{array}{c}\$ 150,206,365- \\
\$ 154,427,041 \\
\end{array}$ & $\begin{array}{c}\$ 151,859,137- \\
\$ 157,076,417 \\
\end{array}$ & $\begin{array}{c}\$ 146,100,651- \\
\$ 146,108,191 \\
\end{array}$ & $\begin{array}{c}\$ 157,651,881- \\
\$ 169,895,270 \\
\end{array}$ \\
\hline ICER undiscounted & & $3,717-7,041$ & $55-128$ & $219-568$ & $114-329$ & $698,391-1,396,623$ & $1124-2231$ \\
\hline $\begin{array}{c}\text { ICER discounted, } \\
3 \% \\
\end{array}$ & & $3,073-6,688$ & $61-114$ & $211-562$ & $105-321$ & $\begin{array}{c}1,248,064- \\
6,241,112 \\
\end{array}$ & $1,077-2,133$ \\
\hline \multicolumn{8}{|c|}{ 2000-Lifetime } \\
\hline $\begin{array}{c}\text { Lifetime QALYs } \\
\text { gained } \\
\text { (undiscounted) }\end{array}$ & & $311,908-679,486$ & $890,257-2,349,725$ & $\begin{array}{c}2,541,849- \\
5,101,261 \\
\end{array}$ & $\begin{array}{c}4,437,466- \\
8,506,243 \\
\end{array}$ & $426-3,408$ & $\begin{array}{l}3,973,407- \\
7,896,584\end{array}$ \\
\hline $\begin{array}{l}\text { Lifetime QALYs } \\
\text { gained }(3 \%) \\
\end{array}$ & & $102,972-224,408$ & $294,824-776,859$ & $846,376-1,699,590$ & $\begin{array}{c}1,477,248- \\
2,841,659 \\
\end{array}$ & $83-1,319$ & $\begin{array}{l}1,486,378- \\
2,971,228\end{array}$ \\
\hline $\begin{array}{l}\text { Lifetime QALYs } \\
\text { gained (10\%) }\end{array}$ & & $26,345-57,290$ & $74,971-196,267$ & $219,869-442,309$ & $382,028-739,634$ & $29-989$ & $\begin{array}{c}1,095,327- \\
2,195,452 \\
\end{array}$ \\
\hline $\begin{array}{l}\text { Total lifetime } \\
\text { healthcare costs } \\
\text { (undiscounted) }\end{array}$ & $\$ 1,677,123,386$ & $\begin{array}{c}\$ 1,693,266,072- \\
\$ 1,707,138,744 \\
\end{array}$ & $\begin{array}{c}\$ 1,706,181,531- \\
\$ 1,749,559,944 \\
\end{array}$ & $\begin{array}{c}\$ 1,778,072,379- \\
\$ 1,880,088,385 \\
\end{array}$ & $\begin{array}{c}\$ 1,825,335,250- \\
\$ 1,894,613,714 \\
\end{array}$ & $\begin{array}{c}\$ 1,677,131,524- \\
\$ 1,677,385,622 \\
\end{array}$ & $\begin{array}{c}\$ 1,930,181,097- \\
\$ 2,101,831,147 \\
\end{array}$ \\
\hline $\begin{array}{c}\begin{array}{c}\text { Total lifetime } \\
\text { healthcare costs } \\
\text { (discounted 3\%) }\end{array} \\
\end{array}$ & $\$ 963,082,381$ & $\begin{array}{l}\$ 970,150,904- \\
\$ 975,826,577 \\
\end{array}$ & $\begin{array}{l}\$ 977,731,379- \\
\$ 999,413,035 \\
\end{array}$ & $\begin{array}{c}\$ 1,017,115,536- \\
\$ 1,071,857,292 \\
\end{array}$ & $\begin{array}{c}\$ 1,038,820,896- \\
\$ 1,072,198,313 \\
\end{array}$ & $\begin{array}{l}\$ 963,089,931- \\
\$ 963,238,009\end{array}$ & $\begin{array}{l}\$ 1,214,586,993- \\
\$ 1,216,140,091 \\
\end{array}$ \\
\hline $\begin{array}{c}\text { Total lifetime } \\
\text { healthcare costs } \\
\text { (discounted 10\%) }\end{array}$ & $\$ 474,489,537$ & $\begin{array}{c}\$ 476,726,776- \\
\$ 478,376,839 \\
\end{array}$ & $\begin{array}{c}\$ 480,092,601- \\
\$ 488,315,023 \\
\end{array}$ & $\begin{array}{c}\$ 497,433,609- \\
\$ 520,776,214\end{array}$ & $\begin{array}{c}\$ 504,232,647- \\
\$ 516,861,325 \\
\end{array}$ & $\begin{array}{c}\$ 474,491,090- \\
\$ 474,606,260\end{array}$ & $\begin{array}{c}\$ 1,075,284,022- \\
\$ 1,151,404,276 \\
\end{array}$ \\
\hline $\begin{array}{l}\text { Lifetime ICER } \\
\text { undiscounted }\end{array}$ & & Cost-saving & Cost-saving & Cost-saving & Cost-saving & $17,287-137,698$ & $67-89$ \\
\hline $\begin{array}{c}\text { Lifetime ICER } \\
\text { discounted, 3\% } \\
\end{array}$ & & $0-55$ & Cost-saving & Cost-saving & Cost-saving & $44,585-706,735$ & $161-237$ \\
\hline $\begin{array}{l}\text { Lifetime ICER } \\
\text { discounted, } 10 \%\end{array}$ & & $154-397$ & Cost-saving & Cost-saving & Cost-saving & $59,422-2,022,518$ & $354-641$ \\
\hline
\end{tabular}

\section{Footnotes for Table 2:}

1. All values, except current conditions, are reported as a range of two numbers, corresponding to two counterfactuals on behavioral parameters in the model that are influenced by the absence of a program: a "Zero" counterfactual, where associated behaviors are kept unchanged and a " $50 \%$ " counterfactual, where 
improvements by $50 \%$ on behavioral parameters associated with the absent program are attributed to unknown effects external to the program. For more on these counterfactual assumptions, see Appendix 5.

2. All cumulative values (number of new infections, number of deaths) are calculated in the period 2000-2010.

3. In Papua, no epidemiological indicators are calculated for MSM, Waria and IDU based on the model assumption that the population sizes of these risk groups in Papua are negligible.

4. Economic evaluations were conducted for two time periods: $2000-2010$ and 2000-Lifetime, where lifetime is assumed as 80 years after ending the simulation at 2010 (hence, 2000-2090).

5. Costs for analyses in the time period 2000-2010 are discounted by $3 \%$. To allow for broader sensitivity analyses in future costs in the time period $2000-$ Lifetime, we use two discount rates (3\% and $10 \%)$.

3. Core prevention programs are those aimed at most-at-risk-populations and general population

4. "Lifetime" QALY, health care costs and ICERs are computed from 2000-2090.

$5 \mathrm{CPI}$ is taken from from http://www.global-rates.com/economic-indicators/inflation/consumer-prices/cpi/indonesia.aspx

6. Some prevention costs not elsewhere classified (NEC) from NASA are reallocated to the core prevention programs

7. The formula to calculate ICERs is the following: incremental costs/lncremental QALYs $=($ (total investment cost + total healthcare costs in current conditions)total healthcare costs without intervention)/(total QALYs in current conditions - total QALYs without intervention)

8. ICERs with negative values are considered 'Cost-saving', since there is decreased cost for increased benefit (QALYs)

9. The ranges for programs aimed at low-risk are taken from the assumption that behavioral parameters are at $90 \%$ to $95 \%$ of values from the status quo (current conditions). 
Impact of Reductions in HIV Prevention Funding and Optimal Allocation of Spending on Prevention Programs

In Figure 5, the distribution of prevention funding is shown according to current allocations and allocations calculated to be optimal for reducing new HIV cases. Optimal allocation according to the model calculations suggest a shift away from funding for the general population and large groups at lower risk and towards MARPs, prioritizing IDUs and consolidating MSM and FSW programs. An optimal allocation of resources is estimated to reduce the overall number of new HIV infections by $19 \%$ compared with current allocations (Figure 6).

Figure 5: Current allocation of HIV prevention funding to programs versus the optimal budget allocation to reduce HIV incidence

Current allocation

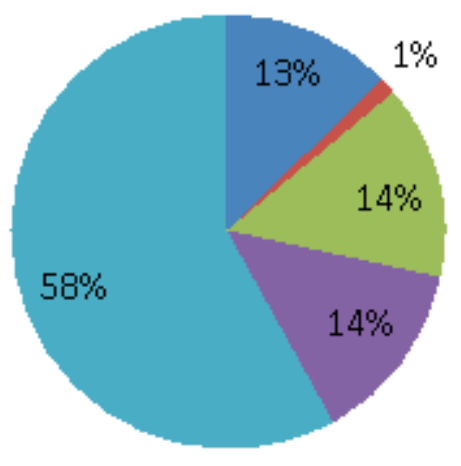

Optimal allocation

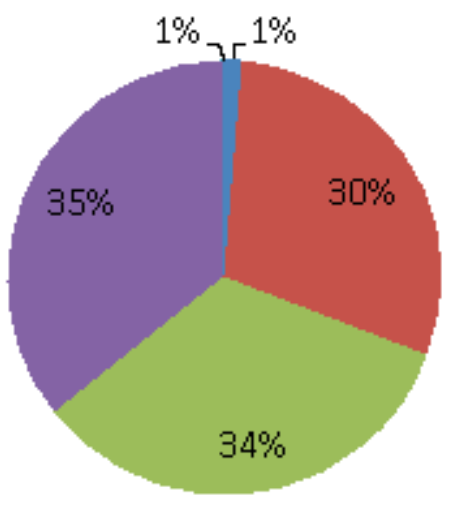

FSW and clients

MSM

NSP

MMT

General

prevention

If there is a reduction in total funds available in the prevention budget, then the optimal allocation will slowly shift (Figure 6). It is observed that when resources are extremely scarce, IDU-targeted programs (particularly NSP) are the first to be funded to a noticeable level and then base MSM programs commence as more funding is available. As further funding is available, MMT programs should be introduced to cover the reachable target population, followed by consolidation and further scale-up of MSM programs and the commencement of scale-up of programs for FSW and their clients. Each program, but particularly FSW programs, should then be scaled up further as more resources become available. 
Figure 6: Optimal allocation of funding to prevention programs for a reduced HIV prevention budget and corresponding incidence in each population group (see Table 3 for details)
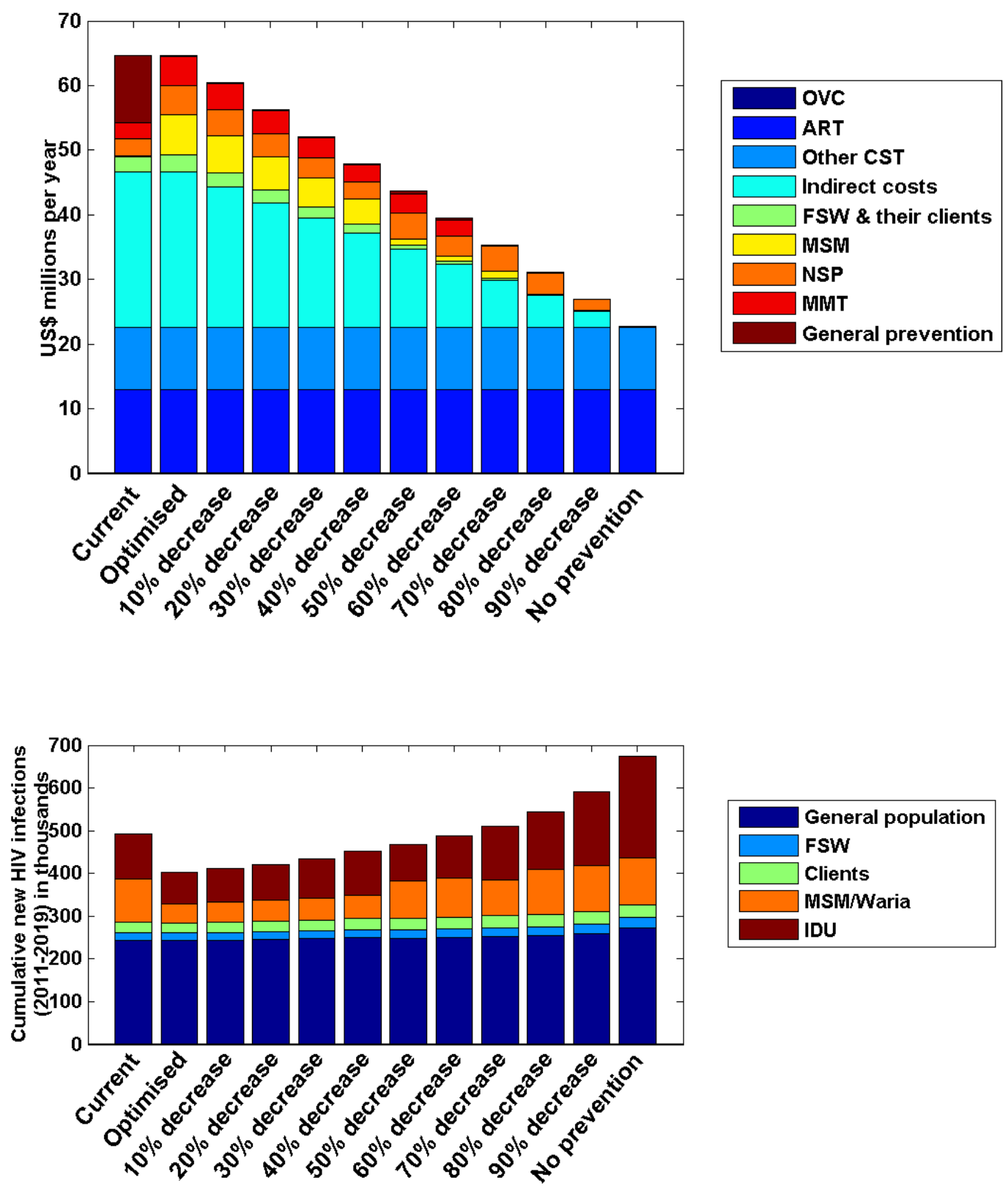

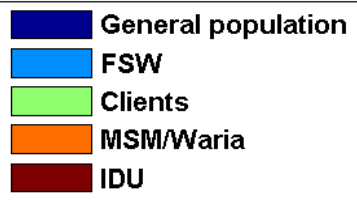


Table 3: HIV outcomes and budget breakdown for reduced 2015-2019 prevention budget

\begin{tabular}{|c|c|c|c|c|c|c|c|c|c|c|c|c|}
\hline \multirow[b]{2}{*}{$\begin{array}{c}\text { Outcomes } 2011- \\
2019\end{array}$} & \multicolumn{2}{|c|}{ Current funding } & \multicolumn{10}{|c|}{ Budget decrease scenarios ( $\%$ decrease) } \\
\hline & $\begin{array}{l}\text { Current } \\
\text { allocation }\end{array}$ & $\begin{array}{l}\text { Optimized } \\
\text { allocation }\end{array}$ & $10 \%$ & $20 \%$ & $30 \%$ & $40 \%$ & $50 \%$ & $60 \%$ & $70 \%$ & $80 \%$ & $90 \%$ & $\begin{array}{c}\text { No } \\
\text { Prevention }\end{array}$ \\
\hline \multicolumn{13}{|c|}{ NON-PAPUA } \\
\hline $\begin{array}{l}\text { Cumulative number } \\
\text { of new infections }\end{array}$ & 424,341 & 326,495 & 332,809 & 340,718 & 350,772 & 363,757 & 380,817 & 403,632 & 432,505 & 466,286 & 514,780 & 600,345 \\
\hline $\begin{array}{l}\text { Cumulative number } \\
\text { of people starting } \\
\text { 1st-line ART }\end{array}$ & 32,488 & 32,503 & 32,503 & 32,502 & 32,500 & 32,498 & 32,495 & 32,490 & 32,487 & 32,482 & 32,468 & 32,434 \\
\hline $\begin{array}{l}\text { Cumulative number } \\
\text { of people starting } \\
\text { 2nd-line ART }\end{array}$ & 2,940 & 2,944 & 2,943 & 2,943 & 2,943 & 2,942 & 2,941 & 2,940 & 2,939 & 2,938 & 2,934 & 2,925 \\
\hline $\begin{array}{l}\text { Cumulative number } \\
\text { of deaths }\end{array}$ & 233,290 & 229,535 & 229,796 & 230,122 & 230,538 & 231,076 & 231,786 & 232,743 & 233,712 & 235,059 & 237,327 & 242,306 \\
\hline \multicolumn{13}{|c|}{ PAPUA } \\
\hline $\begin{array}{l}\text { Cumulative number } \\
\text { of new infections }\end{array}$ & 68,888 & 74,262 & 74,276 & 74,290 & 74,304 & 74,319 & 74,333 & 74,347 & 74,336 & 74,418 & 74,422 & 74,422 \\
\hline $\begin{array}{l}\text { Cumulative number } \\
\text { of people starting } \\
\text { 1st-line ART }\end{array}$ & 1,743 & 1,743 & 1,743 & 1,743 & 1,743 & 1,743 & 1,743 & 1,743 & 1,743 & 1,743 & 1,743 & 1,743 \\
\hline $\begin{array}{l}\text { Cumulative number } \\
\text { of people starting } \\
\text { 2nd-line ART }\end{array}$ & 92 & 92 & 92 & 92 & 92 & 92 & 92 & 92 & 92 & 92 & 92 & 92 \\
\hline $\begin{array}{l}\text { Cumulative number } \\
\text { of deaths }\end{array}$ & 9,465 & 9,580 & 9,581 & 9,581 & 9,581 & 9,581 & 9,582 & 9,582 & 9,582 & 9,583 & 9,583 & 9,583 \\
\hline \multicolumn{13}{|c|}{ Budget allocation per year } \\
\hline OVC & $\$ 64,716$ & $\$ 64,716$ & $\$ 64,716$ & $\$ 64,716$ & $\$ 64,716$ & $\$ 64,716$ & $\$ 64,716$ & $\$ 64,716$ & $\$ 64,716$ & $\$ 64,716$ & $\$ 64,716$ & $\$ 64,716$ \\
\hline ART & $\$ 12,878,514$ & $\$ 12,878,514$ & $\$ 12,878,514$ & $\$ 12,878,514$ & $\$ 12,878,514$ & $\$ 12,878,514$ & $\$ 12,878,514$ & $\$ 12,878,514$ & $\$ 12,878,514$ & $\$ 12,878,514$ & $\$ 12,878,514$ & $\$ 12,878,514$ \\
\hline Other CST & $\$ 9,707,423$ & $\$ 9,707,423$ & $\$ 9,707,423$ & $\$ 9,707,423$ & $\$ 9,707,423$ & $\$ 9,707,423$ & $\$ 9,707,423$ & $\$ 9,707,423$ & $\$ 9,707,423$ & $\$ 9,707,423$ & $\$ 9,707,423$ & $\$ 9,707,423$ \\
\hline Indirect cost & $\$ 24,038,787$ & $\$ 24,039,333$ & $\$ 21,641,025$ & $\$ 19,242,718$ & $\$ 16,844,410$ & $\$ 14,446,103$ & $\$ 12,047,795$ & $\$ 9,649,488$ & $\$ 7,251,180$ & $\$ 4,852,873$ & $\$ 2,454,565$ & $\$ 0$ \\
\hline $\begin{array}{l}\text { FSW and their } \\
\text { clients }\end{array}$ & $\$ 2,265,542$ & $\$ 228,308$ & $\$ 205,477$ & $\$ 182,647$ & $\$ 159,816$ & $\$ 136,985$ & $\$ 114,154$ & $\$ 91,323$ & $\$ 79,913$ & $\$ 8,328$ & $\$ 893$ & $\$ 0$ \\
\hline MSM & $\$ 237,406$ & $\$ 5,333,631$ & $\$ 4,800,268$ & $\$ 4,266,905$ & $\$ 3,733,542$ & $\$ 3,200,179$ & $\$ 2,666,816$ & $\$ 2,133,452$ & $\$ 610,419$ & $\$ 116,922$ & $\$ 8,968$ & $\$ 0$ \\
\hline NSP & $\$ 2,621,065$ & $\$ 6,054,342$ & $\$ 5,448,908$ & $\$ 4,843,474$ & $\$ 4,238,039$ & $\$ 3,632,605$ & $\$ 3,027,171$ & $\$ 2,421,737$ & $\$ 2,774,055$ & $\$ 2,899,807$ & $\$ 1,792,140$ & $\$ 0$ \\
\hline MMT & $\$ 2,442,911$ & $\$ 6,369,428$ & $\$ 5,732,485$ & $\$ 5,095,543$ & $\$ 4,458,600$ & $\$ 3,821,657$ & $\$ 3,184,714$ & $\$ 2,547,771$ & $\$ 1,831,100$ & $\$ 580,143$ & $\$ 551$ & $\$ 0$ \\
\hline Other prevention & $\$ 10,459,788$ & $\$ 40,455$ & $\$ 36,410$ & $\$ 32,364$ & $\$ 28,319$ & $\$ 24,273$ & $\$ 20,228$ & $\$ 16,182$ & $\$ 112,362$ & $\$ 33$ & $\$ 64$ & $\$ 0$ \\
\hline
\end{tabular}


Impact of Flexible ART funding: consideration of treatment as prevention

Investigations were carried out to identify optimal allocations of the HIV/AIDS budget (and reduced funding amounts) across all prevention, treatment and care programs if all funds were flexible for reallocation. These assessments allowed exploration of consideration of antiretroviral treatment for prevention versus care prevention programs, with respect to cost-effectiveness related to minimizing new HIV infections. Increased uptake of ART for treatment-eligible people as well as scenarios of early initiation of ART for people who are diagnosed with HIV with high CD4 counts was investigated (see Figure 7). It was found that greater initiation of ART is not more cost-effective for prevention than traditional prevention programs around harm reduction.

Figure 7: Optimal allocation of funding to HIV prevention programs (including treatment as prevention) to reduce HIV/AIDS related deaths when government contribution increases

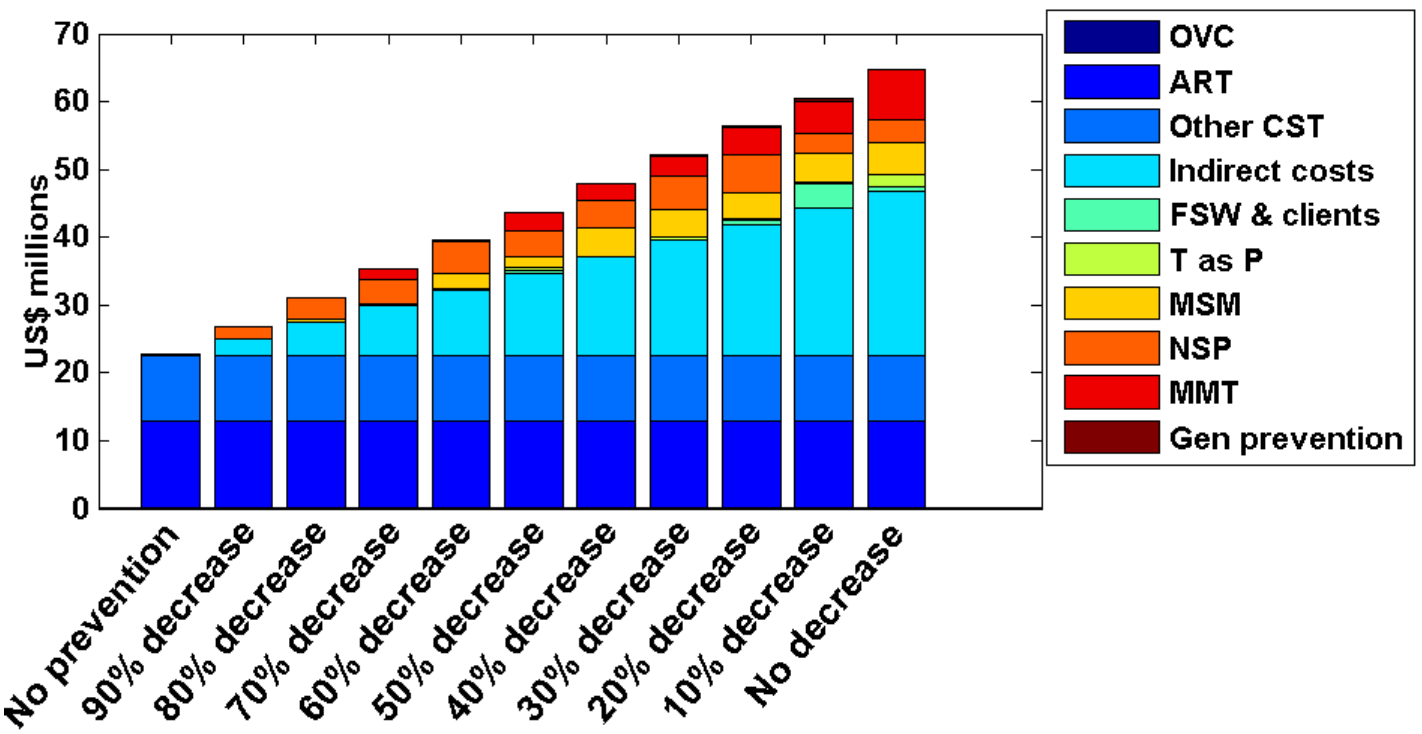

- For all scenarios, the optimal allocation of funding to minimize HIV incidence includes very little additional funding on top of existing treatment budgets allocated towards treatment as prevention.

- However, if the primary objective of the funding is to minimize deaths (or maximize health benefits (QALYs)), and not minimize new infections, then it was found that substantially greater budget allocations should be provided for antiretroviral treatment.

- According to the objective of minimizing new infections, it was identified that an additional $\$ 1.8 \mathrm{~m}$ (2.8\% of the budget) should be allocated to treatment (as prevention).

- Harm reduction programs should have highest priority in low budget scenarios. 
Impact of optimal allocation on HIV prevalence, 2011-2019

In Figure 8, projected prevalence levels are shown by population group according to three scenarios: (i) continued current conditions with no change in budget levels or allocations; (ii) optimal allocation of current budget levels for maximal allocative efficiency at the national level across all populations; (iii) no budget for HIV prevention programs but sustained ART programs.

The epidemic trajectories reveal that the greatest number of new infections occurs among IDUs and that past programs have contributed to stabilizing the epidemic among this group and therefore IDU programs should be prioritized, otherwise it is likely that prevalence could increase markedly from the already high levels. The increase in HIV among FSWs is of high importance but suggestive that past control efforts, while likely to have been somewhat effective in mitigating transmission, have not been as cost-effective as programs targeted at MSM. Therefore, optimal allocations for reducing new infections over 2011-2019 are more targeted to MSM than FSWs. However, FSWs are much more important in acting as a bridge for HIV transmission from MARPs to the general population and so it is important to target these groups to minimize total new infections beyond 2019. In doing so, it is essential that programs targeting FSWs and their clients are made more efficient.

Figure 8: National HIV prevalence in 2000-2019

Low-risk population

- - No prevention

Optimized current budget

Current conditions

Clients of FSWs

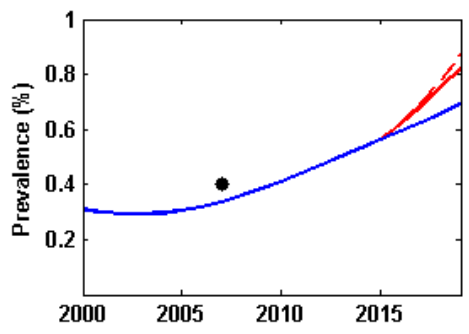

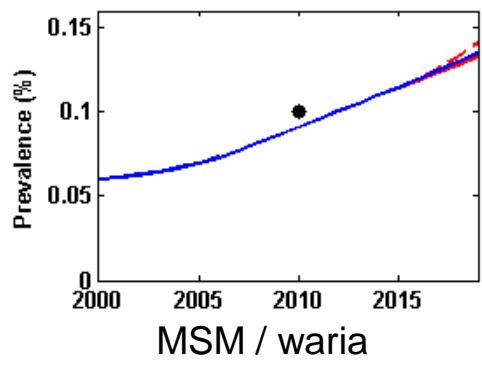

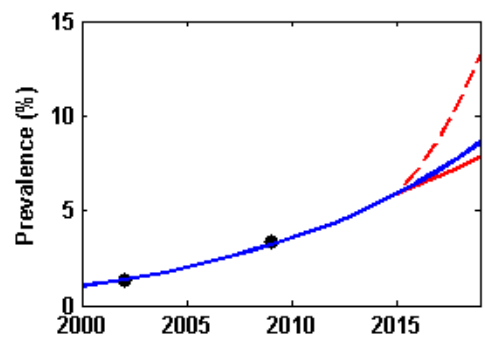

Female sex workers

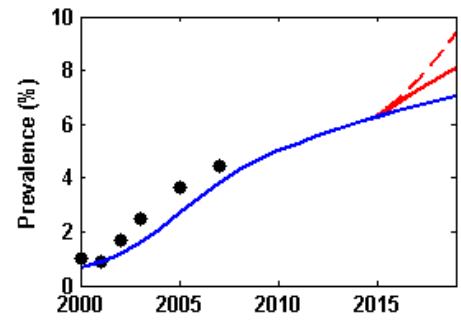

Injecting drug users

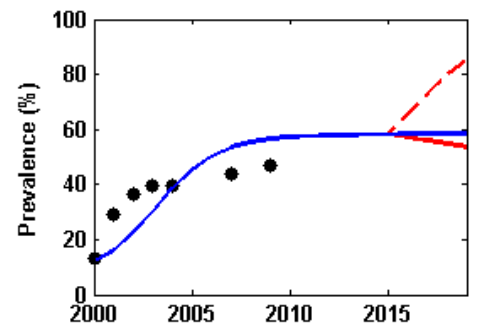




\section{Appendix 1: HIV Spending Data Collation, Estimation of Costs}

Aggregate costs were collected from the UNGASS country reports and corresponding appendices of data from the National AIDS Spending Assessment matrices. These costs were used to estimate the total spending on different HIV/AIDS programs in Indonesia. Table 5 summarizes the total amount spent each year for HIV/AIDS programs. The total spent in the period 2003-2010 is estimated to be US\$363m (adjusted for $\mathrm{CPI}$ ).

Table 6 shows a summarized version of the 2009-2010 breakdown of expenditures for HIV/AIDS in Indonesia, from which the average proportions to assume programmatic costs are computed.

Table 7 summarizes the method for assuming the breakdown of costs for harm reduction expenditure into funds for NSP and MMT (as required in this report), by taking into account the information from the 2007 Costing Study for Harm reduction programs in Indonesia.

Table 8 shows the assumptions made on target populations for specific items in NASA as summarized in table 6, in order to reallocate the substantial amount of "Unknown"/"Unallocated" funds into specific MARPs.

Healthcare costs were provided by the School of Public Health, University of Indonesia. Unit costs for spending items in health care include the cost of antiretroviral therapy (first-line or second-line ART), HIV rapid testing and CD4 cell tests and co-trimoxazole prophylaxis. The frequency of availing services involving these spending items differentiate the total amount spent per year between people on different stages of progression of HIV disease (CD4 levels). Table 9 summarises the unit health care costs used in the analyses. Health utilities used in the analysis are shown in Table 10. 
Table 4: Overall NASA spending totals (available data from NASA, country reports.

\begin{tabular}{cc}
\hline \multicolumn{2}{|c}{ HIV spending } \\
\hline 2003 & $\$ 24,890,908$ \\
\hline 2004 & $\$ 30,407,332$ \\
\hline 2005 & $\$ 64,885,625$ \\
\hline 2006 & $\$ 56,576,587$ \\
\hline 2007 & $\$ 58,671,397$ \\
\hline 2008 & $\$ 49,563,286$ \\
\hline 2009 & $\$ 60,285,420$ \\
\hline 2010 & $\$ 69,146,880$ \\
\hline \multirow{2}{*}{$\begin{array}{c}\text { Total from } \\
2003-2010 \\
\text { with CPI }\end{array}$} & $\$ 362,995,804$ \\
\hline
\end{tabular}


Table 5: Detailed spending breakdown, 2009-2010 NASA data. Average spending Breakdown based on the average spending proportion from the years 2009 and 2010 in NASA.

\begin{tabular}{|c|c|c|c|c|}
\hline & 2009 & 2010 & $\begin{array}{l}\text { average } \\
\text { spending }\end{array}$ & $\begin{array}{l}\% \text { of average } \\
\text { spending }\end{array}$ \\
\hline HIV spending TOTAL overall & $\$ 60,285,420$ & $\$ 69,146,880$ & $\$ 64,716,150$ & \\
\hline Prevention total & $\$ 19,411,106$ & $\$ 20,413,354$ & $\$ 19,912,230$ & $30.77 \%$ \\
\hline Communication for social and behavioral change (BCC) & $\$ 1,731,400$ & $\$ 1,930,280$ & $\$ 1,830,840$ & \\
\hline community mobilization & $\$ 1,092,976$ & $\$ 1,361,603$ & $\$ 1,227,290$ & \\
\hline VCT (non-targeted) & $\$ 189,448$ & $\$ 175,151$ & $\$ 182,300$ & \\
\hline risk reduction for VAP & $\$ 308,564$ & $\$ 656,068$ & $\$ 482,316$ & \\
\hline prevention youth in school & $\$ 452,061$ & $\$ 692,578$ & $\$ 572,320$ & \\
\hline prevention youth out of school & $\$ 320,573$ & $\$ 356,635$ & $\$ 338,604$ & \\
\hline prevention of HIV transmission aimed at PLWH & $\$ 398,001$ & $\$ 600,042$ & $\$ 499,022$ & \\
\hline prevention programs for sex workers and their clients & $\$ 604,968$ & $\$ 1,045,229$ & $\$ 825,099$ & \\
\hline prevention programs for MSM & $\$ 23,211$ & $\$ 149,713$ & $\$ 86,462$ & \\
\hline harm reduction programs for IDUs & $\$ 1,474,163$ & $\$ 2,059,758$ & $\$ 1,766,961$ & \\
\hline prevention programs in the workplace & $\$ 111,176$ & $\$ 93,811$ & $\$ 102,494$ & \\
\hline condom social marketing (non-targeted) & $\$ 4,785$ & $\$ 7,821$ & $\$ 6,303$ & \\
\hline $\begin{array}{l}\text { condom provision by govt. and private sector (non- } \\
\text { targeted) also public and commercial sector male condom } \\
\text { provision (non-targeted) }\end{array}$ & $\$ 35,775$ & $\$ 279,137$ & $\$ 157,456$ & \\
\hline $\begin{array}{l}\text { public and commercial sector female condom provision } \\
\text { (non-targeted) }\end{array}$ & $\$ 164,780$ & $\$ 751,302$ & $\$ 458,041$ & \\
\hline Microbicides & $\$ 0$ & $\$ 0$ & $\$ 0$ & \\
\hline prevention, diagnosis and treatment of STI (non-targeted) & $\$ 140,536$ & $\$ 252,339$ & $\$ 196,438$ & \\
\hline PMTCT & $\$ 107,532$ & $\$ 85,364$ & $\$ 96,448$ & \\
\hline male circumcision & $\$ 0$ & $\$ 0$ & $\$ 0$ & \\
\hline blood safety & $\$ 20,899$ & $\$ 21,651$ & $\$ 21,275$ & \\
\hline safe medical injections & $\$ 27,859$ & $\$ 0$ & $\$ 13,930$ & \\
\hline universal precautions & $\$ 53,760$ & $\$ 16,974$ & $\$ 35,367$ & \\
\hline PEP & $\$ 3,556$ & $\$ 3,556$ & $\$ 3,556$ & \\
\hline
\end{tabular}




\begin{tabular}{|c|c|c|c|c|}
\hline --- not disaggregated by type & $\$ 0$ & $\$ 0$ & $\$ 0$ & \\
\hline --- not elsewhere classified & $\$ 12,145,084$ & $\$ 9,874,342$ & $\$ 11,009,713$ & \\
\hline Care and Treatment & $\$ 21,082,574$ & $\$ 24,120,328$ & $\$ 22,601,451$ & $34.92 \%$ \\
\hline Outpatient care & $\$ 134,289$ & $\$ 261,289$ & $\$ 197,789$ & \\
\hline provided initiated testing and counseling & $\$ 221,700$ & $\$ 1,151,801$ & $\$ 686,751$ & \\
\hline Ol outpatient prophylaxis and treatment & $\$ 129,126$ & $\$ 188,931$ & $\$ 159,029$ & \\
\hline ART & $\$ 11,280,191$ & $\$ 14,494,180$ & $\$ 12,887,186$ & \\
\hline nutritional support associated to ARV therapy & $\$ 79,577$ & $\$ 134,770$ & $\$ 107,174$ & \\
\hline specific HIV-related lab monitoring & $\$ 870,867$ & $\$ 284,424$ & $\$ 577,646$ & \\
\hline dental programs for PLHIV & $\$ 0$ & $\$ 0$ & $\$ 0$ & \\
\hline psychological treatment and support services & $\$ 189,222$ & $\$ 104,228$ & $\$ 146,725$ & \\
\hline outpatient palliative care & $\$ 0$ & $\$ 0$ & $\$ 0$ & \\
\hline \multirow{2}{*}{$\begin{array}{l}\text { home-based care } \\
\text { traditional medicine and informal care and treatment } \\
\text { services }\end{array}$} & $\$ 27,900$ & $\$ 25,922$ & $\$ 26,911$ & \\
\hline & $\$ 39,446$ & $\$ 127,402$ & $\$ 83,424$ & \\
\hline --- not disaggregated by type & $\$ 0$ & $\$ 0$ & $\$ 0$ & \\
\hline --- not elsewhere classified & $\$ 0$ & $\$ 0$ & $\$ 0$ & \\
\hline In-patient care & $\$ 48,927$ & $\$ 84,496$ & $\$ 66,712$ & \\
\hline in-patient treatment of $\mathrm{Ol}$ & $\$ 1,605,076$ & $\$ 1,184,456$ & $\$ 1,394,766$ & \\
\hline in-patient palliative care & $\$ 0$ & $\$ 0$ & $\$ 0$ & \\
\hline --- not disaggregated by type & $\$ 0$ & $\$ 0$ & $\$ 0$ & \\
\hline --- not elsewhere classified & $\$ 0$ & $\$ 0$ & $\$ 0$ & \\
\hline patient transport and emergency response & $\$ 0$ & $\$ 0$ & $\$ 0$ & \\
\hline --- not disaggregated by type & $\$ 0$ & $\$ 0$ & $\$ 0$ & \\
\hline --- not elsewhere classified & $\$ 6,456,251$ & $\$ 6,078,428$ & $\$ 6,267,340$ & \\
\hline Orphans and vulnerable children & $\$ 82,261$ & $\$ 128,658$ & $\$ 105,460$ & $0.16 \%$ \\
\hline OVC education & $\$ 39$ & $\$ 0$ & $\$ 20$ & \\
\hline OVC basic health and care & $\$ 0$ & $\$ 881$ & $\$ 441$ & \\
\hline OVC family and home support & $\$ 0$ & $\$ 0$ & $\$ 0$ & \\
\hline OVC community support & $\$ 82,222$ & $\$ 127,778$ & $\$ 105,000$ & \\
\hline OVC social services and admin costs & $\$ 0$ & $\$ 0$ & $\$ 0$ & \\
\hline
\end{tabular}




\begin{tabular}{|c|c|c|c|c|}
\hline OVC institutional care & $\$ 0$ & $\$ 0$ & $\$ 0$ & \\
\hline --- not disaggregated by type & $\$ 0$ & $\$ 0$ & $\$ 0$ & \\
\hline --- not elsewhere classified & $\$ 0$ & $\$ 0$ & $\$ 0$ & \\
\hline $\begin{array}{l}\text { Program management and administrative } \\
\text { strengthening }\end{array}$ & $\$ 9,162,412$ & $\$ 13,405,850$ & $\$ 11,284,131$ & $17.44 \%$ \\
\hline planning, coordination and program management & $\$ 2,418,606$ & $\$ 2,732,918$ & $\$ 2,575,762$ & \\
\hline management program & $\$ 1,460,585$ & $\$ 1,366,459$ & $\$ 1,413,522$ & \\
\hline planning and coordination & $\$ 958,021$ & $\$ 1,366,459$ & $\$ 1,162,240$ & \\
\hline \multicolumn{5}{|l|}{$\begin{array}{l}\text { Administration and transaction costs associated with } \\
\text { managing and disbursing funds }\end{array}$} \\
\hline Monitoring and evaluation & $\$ 827,481$ & $\$ 1,377,396$ & $\$ 1,102,439$ & \\
\hline Operations research & $\$ 6,058$ & $\$ 731$ & $\$ 3,395$ & \\
\hline Serological-surveillance & $\$ 68,811$ & $\$ 204,786$ & $\$ 136,799$ & \\
\hline HIV DR surveillance & $\$ 0$ & $\$ 0$ & $\$ 0$ & \\
\hline Drug supply systems & $\$ 834,721$ & $\$ 59,820$ & $\$ 447,271$ & \\
\hline information technology & $\$ 74,886$ & $\$ 256,999$ & $\$ 165,943$ & \\
\hline supervision of personnel & $\$ 103,685$ & $\$ 161,448$ & $\$ 132,567$ & \\
\hline \multicolumn{5}{|l|}{ patient tracking } \\
\hline upgrading and construction of infrastructure & $\$ 22,503$ & $\$ 35,636$ & $\$ 29,070$ & \\
\hline new health facilities & $\$ 56$ & $\$ 48,889$ & $\$ 24,473$ & \\
\hline \multicolumn{5}{|l|}{$\begin{array}{l}\text { mandatory HIV testing } \\
\text {--- not disaggregated by type }\end{array}$} \\
\hline -- not elsewhere classified & $\$ 4,805,606$ & $\$ 4,871,764$ & $\$ 4,838,685$ & \\
\hline Human resources & $\$ 1,528,423$ & $\$ 3,132,356$ & $\$ 2,330,390$ & $3.60 \%$ \\
\hline \multicolumn{5}{|l|}{ Monetary incentives for HR } \\
\hline physicians & $\$ 600$ & $\$ 617$ & $\$ 609$ & \\
\hline nurses & $\$ 6,178$ & $\$ 6,960$ & $\$ 6,569$ & \\
\hline other staff & $\$ 235,617$ & $\$ 1,316,925$ & $\$ 776,271$ & \\
\hline Formative education to build up an HIV workforce & $\$ 18,679$ & $\$ 2,056$ & $\$ 10,368$ & \\
\hline Training & $\$ 749,205$ & $\$ 1,421,531$ & $\$ 1,085,368$ & \\
\hline
\end{tabular}




\begin{tabular}{|c|c|c|c|c|}
\hline --- not elsewhere classified & $\$ 225,374$ & $\$ 384,267$ & $\$ 304,821$ & \\
\hline Social protection and social services excluding OVC & $\$ 243,101$ & $\$ 134,091$ & $\$ 188,596$ & $0.29 \%$ \\
\hline Social protection through monetary benefits & $\$ 16,767$ & $\$ 30,278$ & $\$ 23,523$ & \\
\hline social protection through in-kind benefits & $\$ 10,667$ & $\$ 556$ & $\$ 5,612$ & \\
\hline social protection through provision of social services & $\$ 42,472$ & $\$ 46,464$ & $\$ 44,468$ & \\
\hline HIV-specific income generation projects & $\$ 81,994$ & $\$ 15,022$ & $\$ 48,508$ & \\
\hline --- not disaggregated by type & $\$ 0$ & & $\$ 0$ & \\
\hline --- not elsewhere classified & $\$ 91,201$ & $\$ 41,771$ & $\$ 66,486$ & \\
\hline Enabling environment & $\$ 8,017,047$ & $\$ 6,886,595$ & $\$ 7,451,821$ & $11.51 \%$ \\
\hline advocacy & $\$ 607,585$ & $\$ 475,837$ & $\$ 541,711$ & \\
\hline Human rights programmes & $\$ 221,281$ & $\$ 0$ & $\$ 110,641$ & \\
\hline AIDS specific institutional development & $\$ 1,750,670$ & $\$ 1,964,302$ & $\$ 1,857,486$ & \\
\hline AIDS-specific programmes focused on women & $\$ 19,504$ & $\$ 92,805$ & $\$ 56,155$ & \\
\hline Programmes to reduce gender-based violence & $\$ 0$ & & $\$ 0$ & \\
\hline --- not disaggregated by type & $\$ 0$ & & $\$ 0$ & \\
\hline --- not elsewhere classified & $\$ 5,418,007$ & $\$ 4,353,651$ & $\$ 4,885,829$ & \\
\hline Research & $\$ 758,495$ & $\$ 925,649$ & $\$ 842,072$ & $1.30 \%$ \\
\hline Biomedical research & $\$ 0$ & $\$ 0$ & $\$ 0$ & \\
\hline Clinical research & $\$ 0$ & $\$ 0$ & $\$ 0$ & \\
\hline Epidemiological research & $\$ 2,296$ & $\$ 0$ & $\$ 1,148$ & \\
\hline Social science research & $\$ 77,916$ & $\$ 77,833$ & $\$ 77,875$ & \\
\hline behaviour & $\$ 27,461$ & $\$ 187,000$ & $\$ 107,231$ & \\
\hline economics & $\$ 188,728$ & $\$ 201,444$ & $\$ 195,086$ & \\
\hline strengthening capacity & $\$ 0$ & $\$ 1,100$ & $\$ 550$ & \\
\hline Vaccine-related research & $\$ 0$ & $\$ 0$ & $\$ 0$ & \\
\hline --- not disaggregated by type & $\$ 0$ & & $\$ 0$ & \\
\hline --- not elsewhere classified & $\$ 462,094$ & $\$ 458,272$ & $\$ 460,183$ & \\
\hline
\end{tabular}


Table 6: Proportion of total HIV spending prioritized to each IDU harm reduction category

\begin{tabular}{|c|c|c|c|}
\hline & Harm Reduction Category & Total cost $2007^{\star}$ (US\$) & $\%$ \\
\hline Group 1 & IEC, outreach, peer education, BCC & 331,779 & $29.36 \%$ \\
\hline Group 2 & RDC, VCT, CST and primary health services & 269,563 & $23.86 \%$ \\
\hline Group 3 & NSP and safe disposal (includes injecting equipment and condoms) & 278,666 & $24.66 \%$ \\
\hline Group 4 & drug dependent therapy & 12,775 & $1.13 \%$ \\
\hline Group 5 & Clinical-based substitution therapy, including MMT & 237,174 & $20.99 \%$ \\
\hline Total & & $1,129,957$ & \\
\hline \multicolumn{4}{|c|}{$\begin{array}{l}{ }^{*} \text { These spendir } \\
\text { only for } 4 \text { provin } \\
\text { * We take the pr }\end{array}$} \\
\hline \multicolumn{4}{|c|}{ Assumptions: } \\
\hline \multicolumn{4}{|c|}{ 1. DDT assumed to have no impact, hence reallocated to prevention spending aimed at general population. } \\
\hline \multicolumn{4}{|c|}{$\begin{array}{l}\text { 2. BCC, VCT and NSP assumed to have impact on IDU condom use, partner numbers, testing, syringe sharing and } \% \text { IDUs who } \\
\text { share. }\end{array}$} \\
\hline \multicolumn{4}{|c|}{ 3. Clinical-based substitution therapy assumed to represent MMT. } \\
\hline 4. $M$ & sumed to have impact on average number of injections and \% IDUs & MMT & \\
\hline
\end{tabular}


Table 7: Breakdown of NASA prevention items and prioritized populations

\begin{tabular}{|c|c|c|c|}
\hline Prevention spending item & Prioritized population & $\begin{array}{l}\% \text { of } \\
\text { prevention } \\
\text { spending }\end{array}$ & $\begin{array}{l}\% \text { of all } \\
\text { HIV/AIDS } \\
\text { spending }\end{array}$ \\
\hline Communication for social and behavioral change (BCC) & General population & $9.19 \%$ & $2.83 \%$ \\
\hline Community mobilization & General population & $6.16 \%$ & $1.90 \%$ \\
\hline VCT (non-targeted) & General population & $0.92 \%$ & $0.28 \%$ \\
\hline Risk reduction for VAP & General population & $2.42 \%$ & $0.75 \%$ \\
\hline Prevention youth in school & General population & $2.87 \%$ & $0.88 \%$ \\
\hline Prevention youth out of school & General population & $1.70 \%$ & $0.52 \%$ \\
\hline Prevention of HIV transmission aimed at PLWH & $\begin{array}{l}\text { PLWH/STI/PMTCT/PEP } \\
\text { (Reallocated to MARPs) }\end{array}$ & $2.51 \%$ & $0.77 \%$ \\
\hline Prevention programs for sex workers and their clients & FSW/Clients & $4.14 \%$ & $1.27 \%$ \\
\hline Prevention programs for MSM & MSM & $0.43 \%$ & $0.13 \%$ \\
\hline $\begin{array}{l}\text { Group 1: information, education, communication; outreach; peer } \\
\text { educators (BCC) }\end{array}$ & IDUs: NSP & $2.61 \%$ & $0.80 \%$ \\
\hline $\begin{array}{l}\text { Group 2: risk reduction care; VCT; care support and treatment; } \\
\text { primary health services }\end{array}$ & IDUs: NSP & $2.12 \%$ & $0.65 \%$ \\
\hline Group 3: NSEP; safe disposal & IDUs: NSP & $2.19 \%$ & $0.67 \%$ \\
\hline Group 4: drug dependent therapy & $\begin{array}{l}\text { DDT (Reallocated to } \\
\text { general population) }\end{array}$ & $0.10 \%$ & $0.03 \%$ \\
\hline Group 5: clinical based substitution therapy (methadone) & IDUs: MMT & $1.86 \%$ & $0.57 \%$ \\
\hline Prevention programs in the workplace & General population & $0.51 \%$ & $0.16 \%$ \\
\hline
\end{tabular}




\begin{tabular}{|c|c|c|c|}
\hline Condom social marketing (non-targeted) & General population & $0.03 \%$ & $0.01 \%$ \\
\hline Condom provision by govt. and private sector (non-targeted) & General population & $0.79 \%$ & $0.24 \%$ \\
\hline $\begin{array}{l}\text { Public and commercial sector female condom provision (non- } \\
\text { targeted) also labeled public and commercial sector male } \\
\text { condom provision (non-targeted) }\end{array}$ & General population & $2.30 \%$ & $0.71 \%$ \\
\hline Microbicides & No allocation & $0.00 \%$ & $0.00 \%$ \\
\hline Prevention, diagnosis and treatment of STI (non-targeted) & $\begin{array}{l}\text { PLWH/STI/PMTCT/PEP } \\
\text { (Reallocated to MARPs) }\end{array}$ & $0.99 \%$ & $0.30 \%$ \\
\hline PMTCT & $\begin{array}{l}\text { PLWH/STI/PMTCT/PEP } \\
\text { (Reallocated to MARPs) }\end{array}$ & $0.48 \%$ & $0.15 \%$ \\
\hline Male circumcision & No allocation & $0.00 \%$ & $0.00 \%$ \\
\hline Blood safety & $\begin{array}{l}\text { Health systems (Reallocated } \\
\text { to } \\
\text { indirect cost to prevention) }\end{array}$ & $0.11 \%$ & $0.03 \%$ \\
\hline Safe medical injections & $\begin{array}{l}\text { Health systems (Reallocated } \\
\text { to } \\
\text { indirect cost to prevention) }\end{array}$ & $0.07 \%$ & $0.02 \%$ \\
\hline Universal precautions & $\begin{array}{l}\text { Health systems (Reallocated } \\
\text { to } \\
\text { indirect cost to prevention) }\end{array}$ & $0.18 \%$ & $0.05 \%$ \\
\hline PEP & $\begin{array}{l}\text { PLWH/STI/PMTCT/PEP } \\
\text { (Reallocated to MARPs) }\end{array}$ & $0.02 \%$ & $0.01 \%$ \\
\hline Not elsewhere classified & $\begin{array}{l}\text { Unknown (Reallocated to } \\
\text { general population and } \\
\text { MARPs) }\end{array}$ & $55.29 \%$ & $17.01 \%$ \\
\hline
\end{tabular}


Figure 1: Allocation of prevention spending (2009-2010 NASA average) on programs for prioritized populations

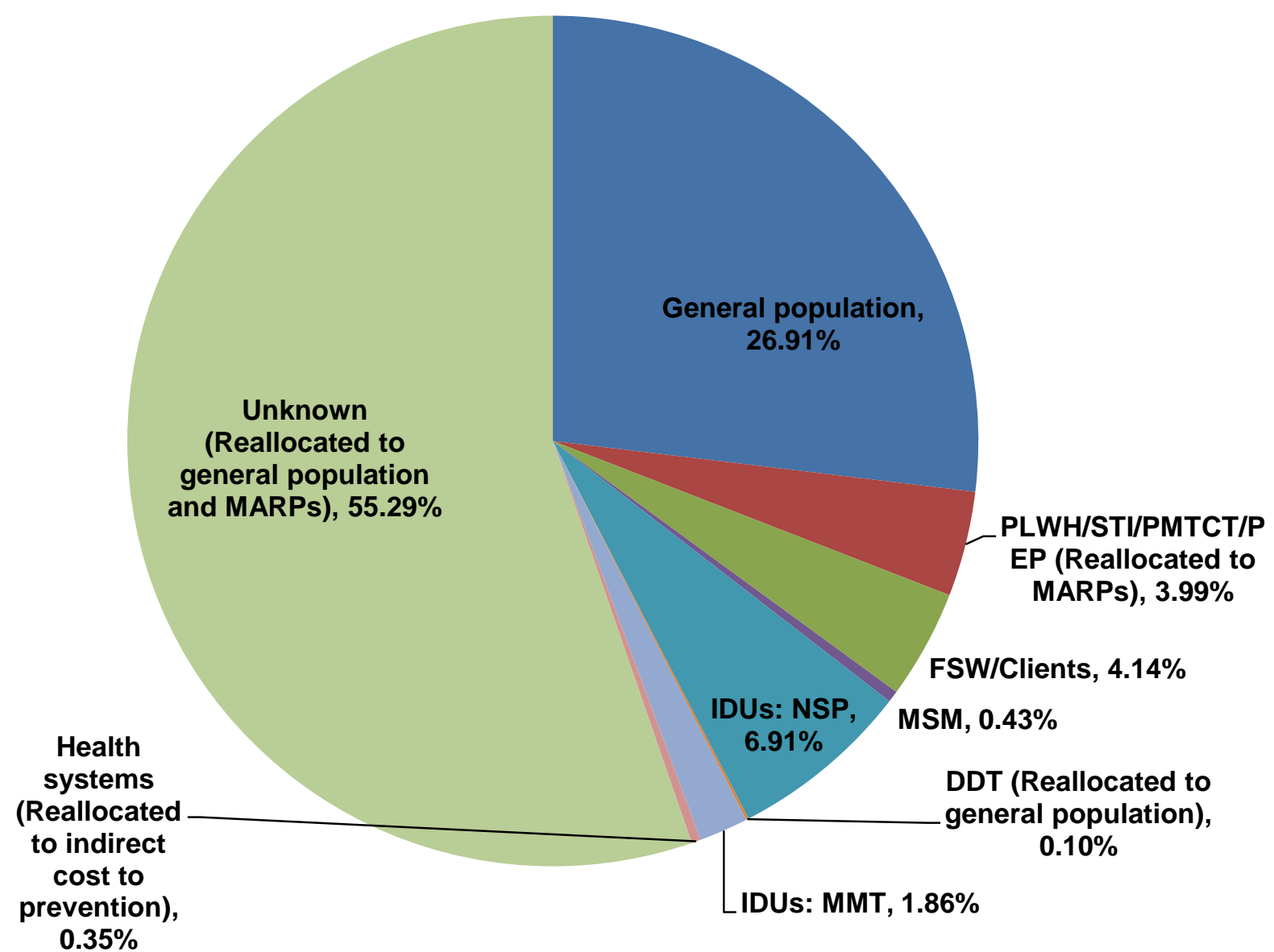


Table 8: Healthcare costs of HIV infected people (annual cost per person in 2012 \$US dollars)*

\begin{tabular}{|c|c|c|}
\hline Stage of HIV Infection & Annual Cost & Reference \\
\hline PLHIV who have CD 4 count $>500$ cells per $\mu \mathrm{l}$ & US\$27 & Correspondence with $\mathrm{MOH}$ \\
\hline PLHIV who have CD4 count $350-500$ cells per $\mu \mathrm{l}$ & US\$27 & Correspondence with $\mathrm{MOH}$ \\
\hline PLHIV who have CD4 count $200-350$ cells per $\mu \mathrm{l}$ & US\$27 & Correspondence with $\mathrm{MOH}$ \\
\hline PLHIV who have CD4 count $<200$ cells per $\mu \mathrm{l}$ & US\$27 & Correspondence with $\mathrm{MOH}$ \\
\hline Cost of first-line ART & US $\$ 453.6$ & Correspondence with $\mathrm{MOH}$ \\
\hline Cost of second-line ART & US $\$ 2138.4$ & Correspondence with $\mathrm{MOH}$ \\
\hline Non-ART healthcare costs & US\$37 & Correspondence with $\mathrm{MOH}$ \\
\hline HIV diagnosis (Rapid test) & US $\$ 1.08$ & Correspondence with $\mathrm{MOH}$ \\
\hline
\end{tabular}


Table 9: Health utilities for cost effectiveness calculations

\begin{tabular}{|lcc|}
\hline Health Utility Parameters & Lower Estimate & Upper Estimate \\
\hline Health Utility of uninfected IDU & 0.93 & 0.96 \\
Health Utility of PLHIV with CD4 > 500 & 0.84 & 0.95 \\
Health Utility of PLHIV with CD4 is 350-500 & 0.84 & 0.93 \\
Health Utility of PLHIV with CD4 is 200-350 & 0.72 & 0.93 \\
Health Utility of PLHIV with CD4 < 200 & 0.60 & 0.85 \\
Health Utility of PLHIV on ART & 0.70 & 0.90 \\
\hline
\end{tabular}




\section{Appendix 2: Model of HIV transmission in Indonesia}

We investigated the cost effectiveness of HIV prevention programs in Indonesia and the impact of changes in HIV funding using a detailed mathematical model of HIV transmission. Relating the changes in funding to the appropriate transmission parameters in the model, we calculated the change in HIV incidence, the number of HIV/AIDS deaths and cost-effectiveness of HIV prevention programs. To do this we used a modified version of the previously developed HIV in Indonesia Model (HIM). Previously, this model was used to investigate the impact of HIV prevention programs in eight regions of Indonesia. A detailed description of the model is available elsewhere. Here we provide a brief summary.

Informed by available HIV surveillance data the model divides the 15-49 year old into 10 distinct population groups for each region in Indonesia.

- Male and female injecting drug users (IDU)

- $\quad$ Direct and indirect female sex workers (FSW)

- Clients of FSW

- Waria

- Bisexual men

- $\quad$ Men who have sex with men (MSM)

- Low risk males and females in the general population

HIM was specifically designed for Indonesia using best-practice HIV epidemic modeling techniques incorporating realistic biological transmission processes, detailed infection progression, and sexual mixing patterns and drug injection behaviors. Through a set of ordinary differential equations, the model tracks HIV transmission and the number of HIV positive people in the non-Papua and Papuan populations and their rate of disease progression via CD4 count. HIM also records deaths due to HIV/AIDS or other causes. The model distinguishes people who are undiagnosed, diagnosed, and on effective anti-retroviral therapy (ART) as shown in Figure 9. HIV transmission within the population occurs through the interaction between different population groups. HIV infections occur through regular, casual or commercial sexual partnerships or through sharing of injecting equipment as shown in 'Model schematics for HIV infection progression and transmission. 


\section{Model schematics for HIV infection progression and transmission}

HIV Infection Progression

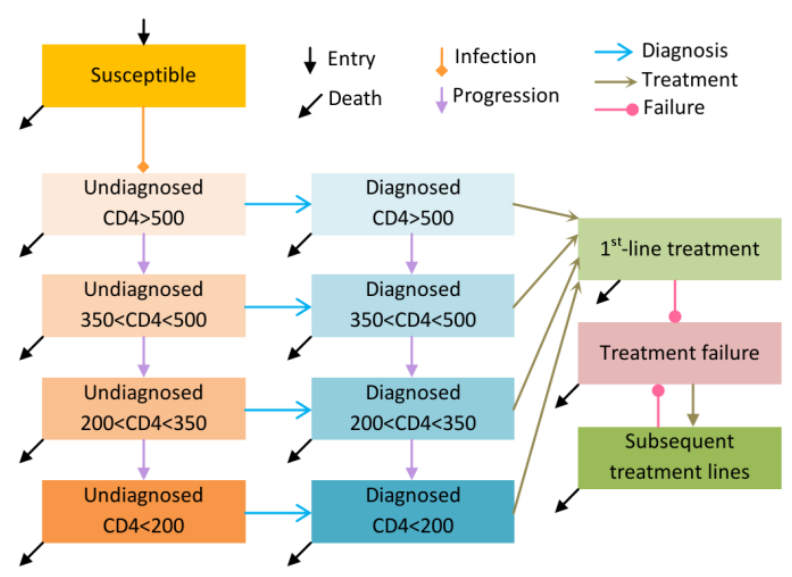

HIV Transmission

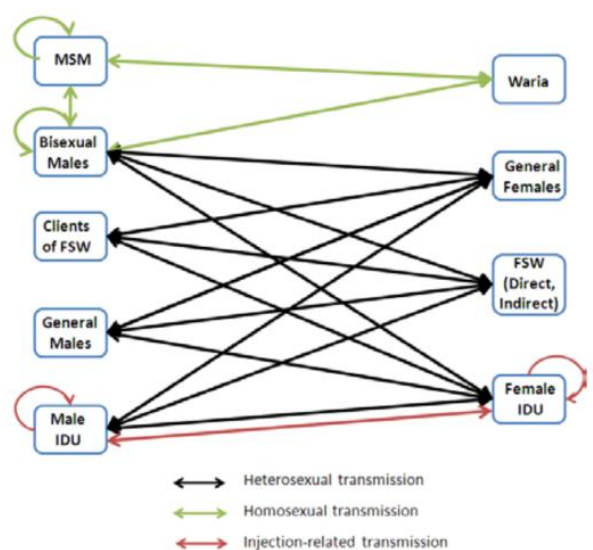

Sexual transmission depends on the prevalence of HIV, the number of casual and regular homosexual and heterosexual partnerships per person, the frequency of sexual acts within a partnership, condom usage, and the infection stage of HIV-positive partners. For IDUs intravenous transmission is dependent on number of injecting partners, frequency of injecting, frequency of sharing equipment, cleaning of syringes, and the efficacy of cleaning. These factors are incorporated into risk-equations within the model to determine the annual per-capita risk of a susceptible person becoming infected with HIV. HIM describes the impact of HIV prevention programs indirectly through their influence on behavioral, clinical, and injecting parameters. Methadone maintenance programs (MMT) are explicitly incorporated into the model.

Indonesia has a wide diversity of HIV epidemics meaning that applying HIM to the overall Indonesian population is inappropriate. Ideally, HIM would be applied to each region in Indonesia to accurately match their HIV epidemics. However, HIV spending and funding is only available at the national level and cannot be broken down to the regional level. We balanced the desire to describe Indonesian epidemics accurately and available funding data by applying HIM to the non-Papuan population, where HIV epidemics are concentrated in at-risk populations, and Papua, where the HIV epidemic is more generalized and primarily driven by sex work.

Model input parameters for non-Papua and Papua populations were informed by all available behavioral data regarding sexual or injecting risk activities, biological data on disease progression and heterogeneous transmission rates, and clinical data (such as rates of VCT and antiretroviral coverage). Any data from 200-2010 were used as inputs, where data was not available assumptions were made based on consultations with Indonesian stakeholders.

To calibrate HIM to the HIV epidemics in non-Papua and Papua, all parameters were first assigned a best estimate with uncertainty bounds. The model was then calibrated using gradient-descent optimization to identify parameter values that yielded epidemic projections which matched available population-level epidemiological data from 2000-2010 including HIV prevalence in each population group and the number of people on ART.

Data to inform HIM are presented in the accompanying Indonesia Data Triangulation Report. 


\section{Appendix 3: Calibration of model to the HIV epidemics in non-Papua and Papua}

HIM was calibrated to HIV prevalence in each population group in non-Papua and Papua over 2000-2010. While matching HIV prevalence, HIM optimized input parameters to available behavioral data for this period as best as possible.

The figures below show the output HIV prevalence for each population group (blue lines) compared to prevalence data (black discs). The corresponding calibrated parameters for each behavioral factor are shown in the Appendix 5: Counterfactuals used in .

The calibrated input parameters and resulting model simulation represent the baseline conditions for the 2000 to 2010 period. The values of each parameter in 2010 represent current conditions.

\section{Non-Papua}

Low Risk Males

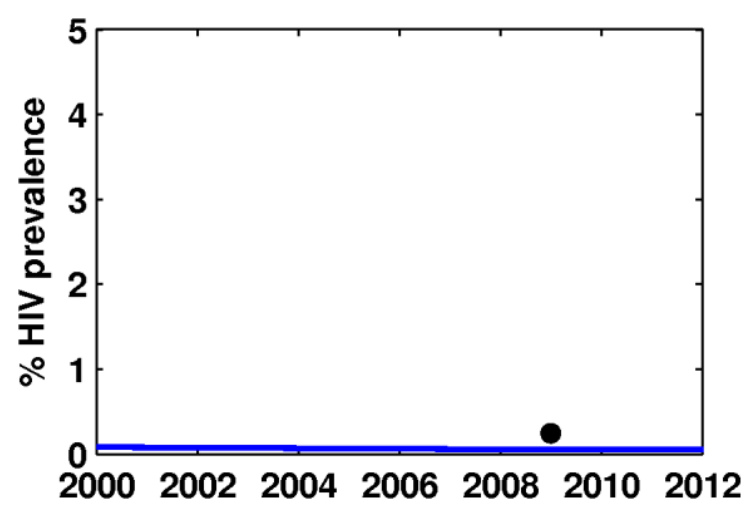

Direct FSW

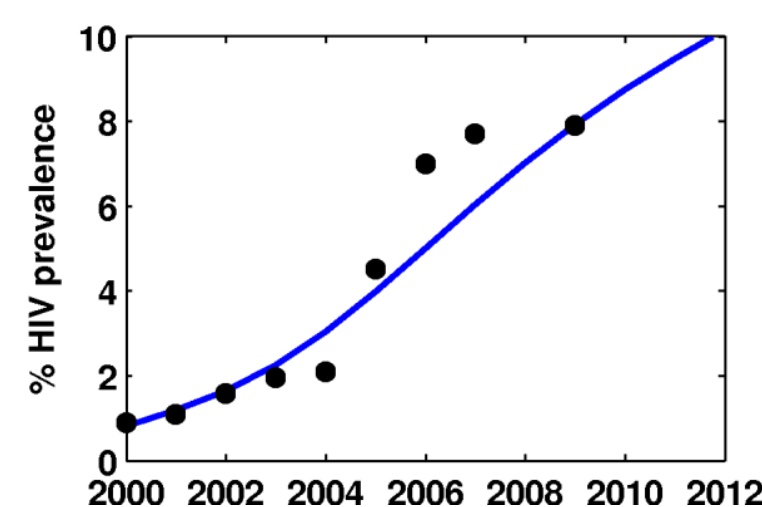

Low Risk Females

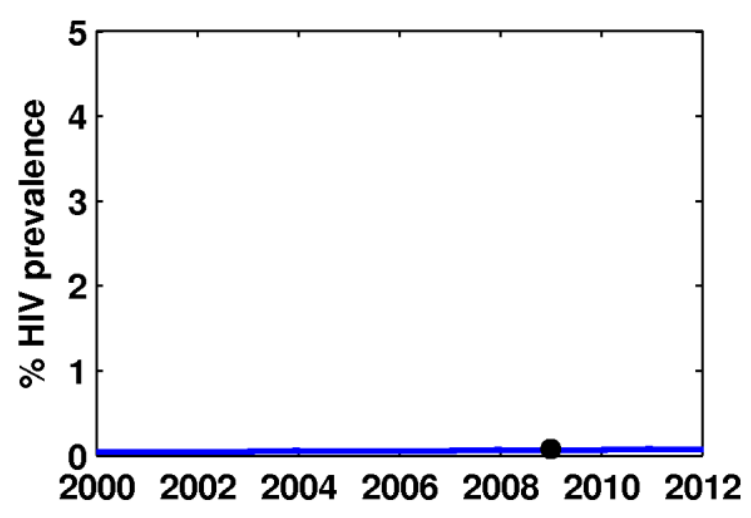

Indirect FSW

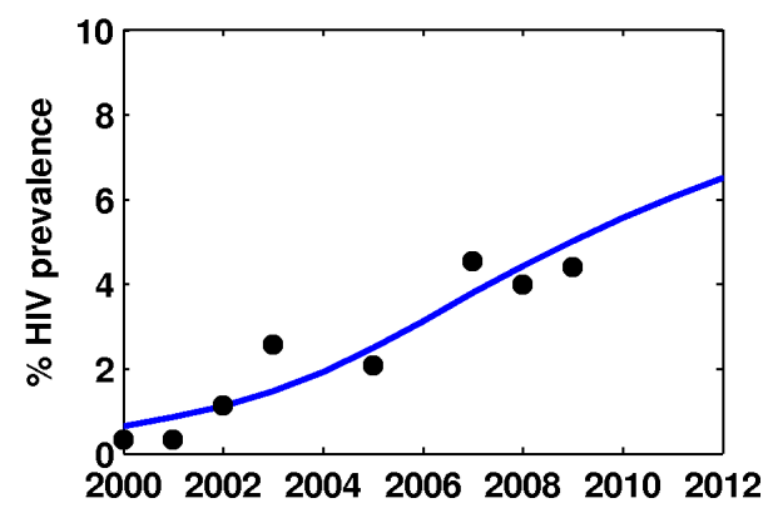


Clients of FSW

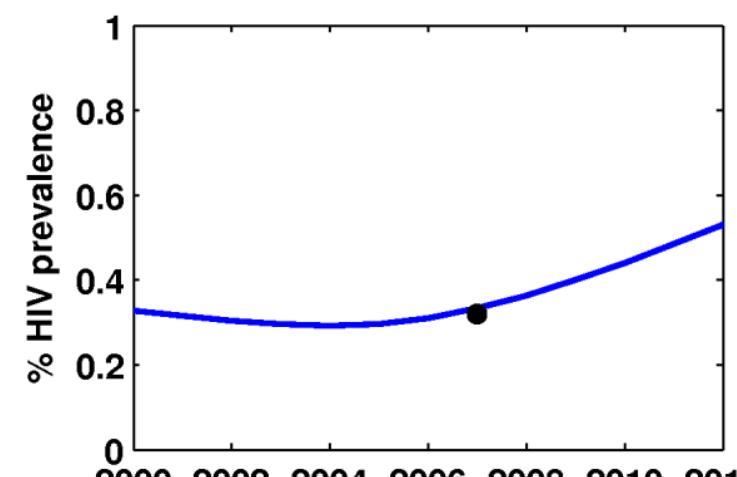

2000200220042006200820102012

Waria

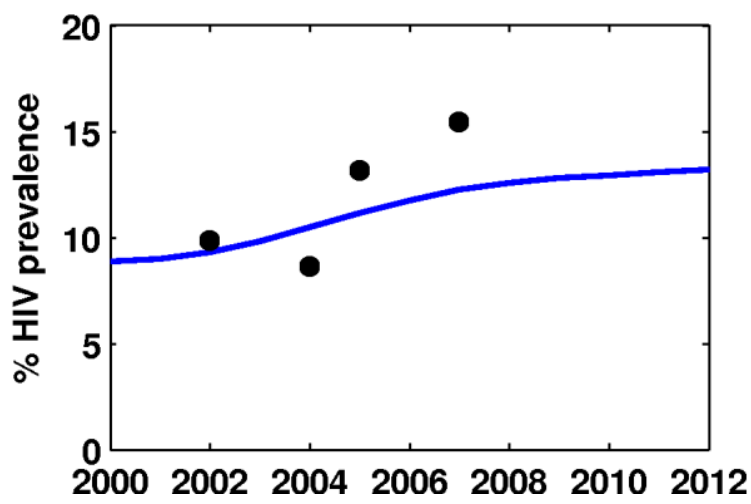

MSM
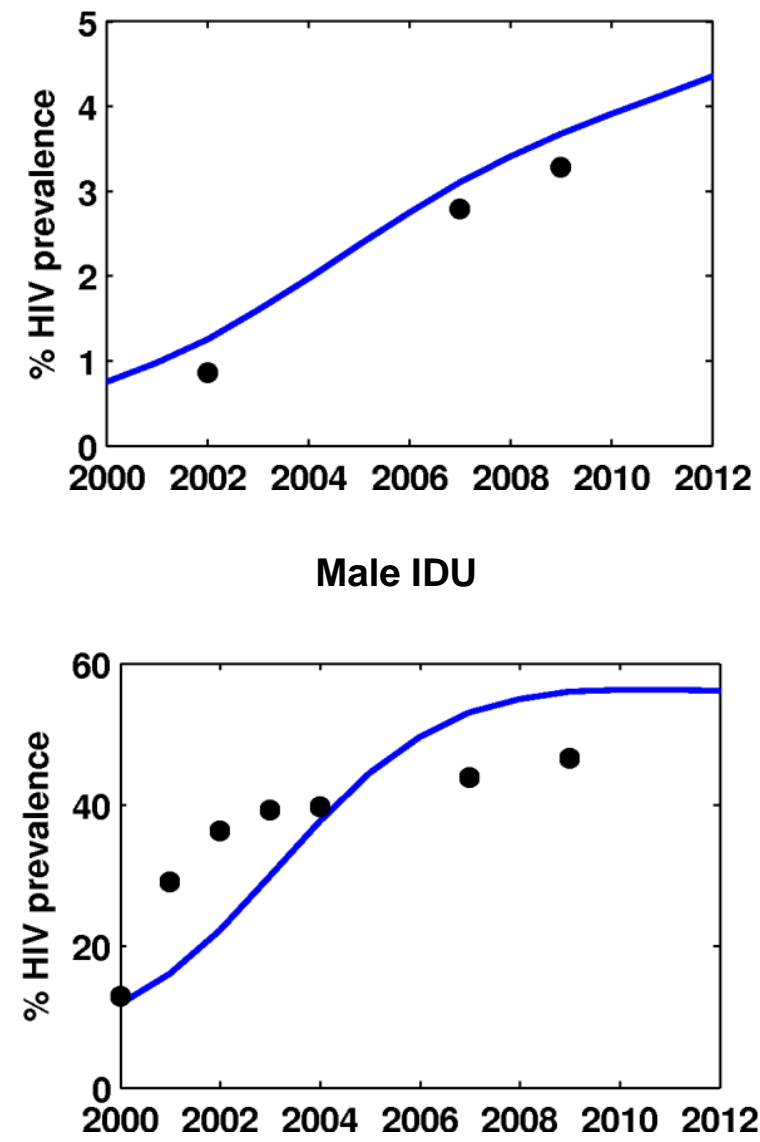

Female IDU

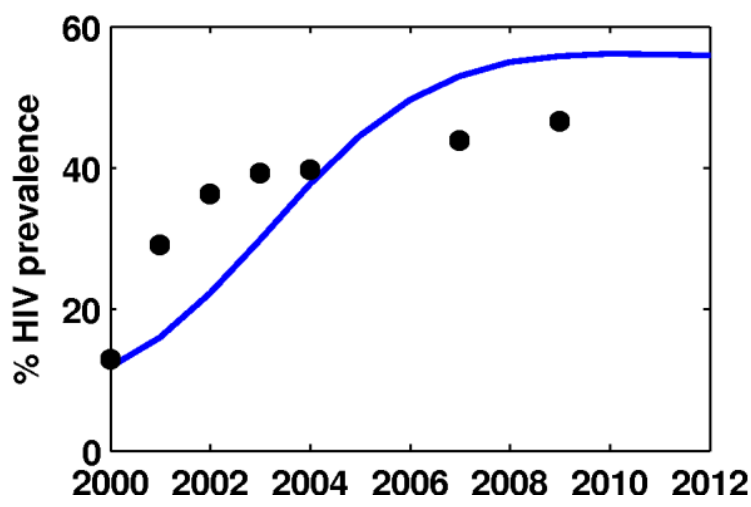




\section{Overall prevalence in Non-Papua}

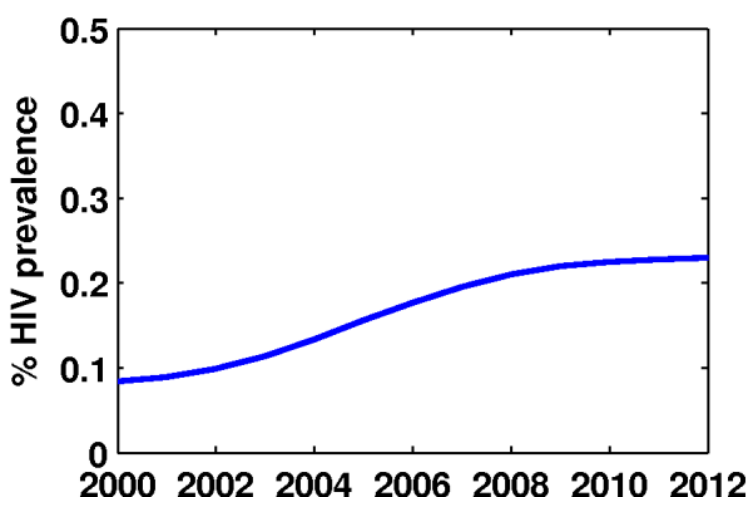

\section{Papua}

Low Risk Males

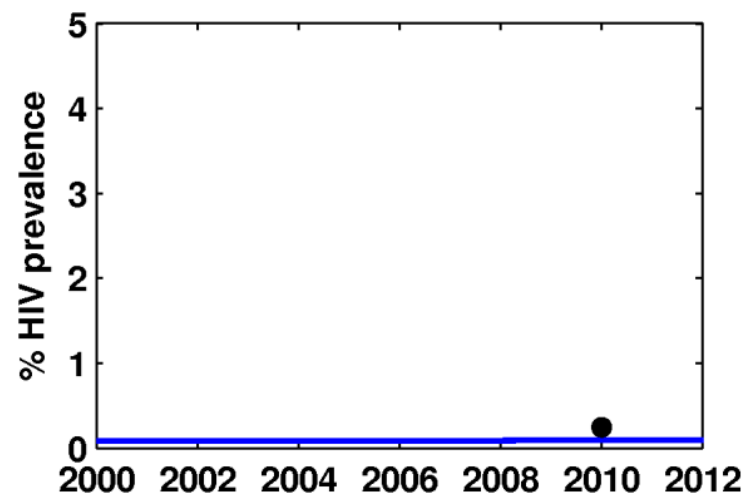

Direct FSW

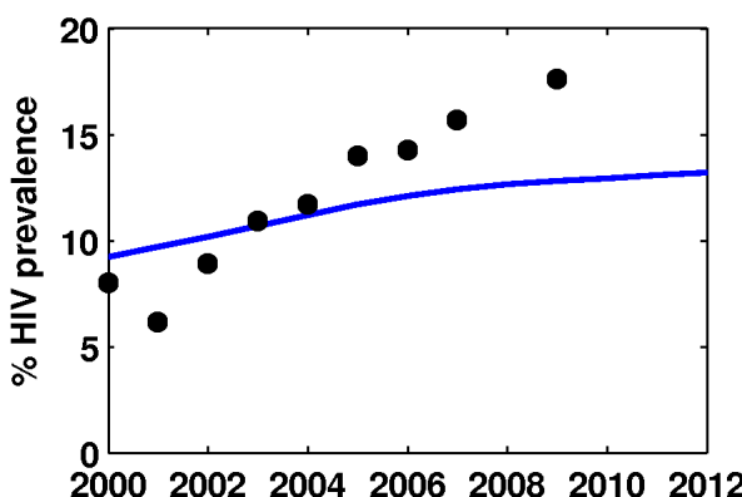

Low Risk Females

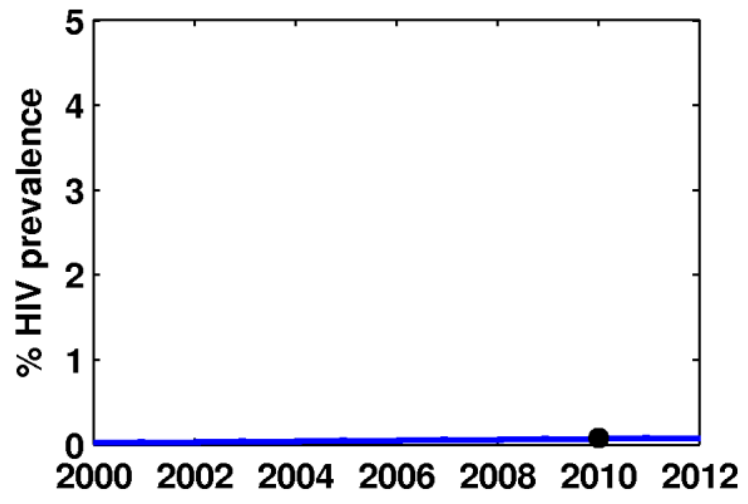

Indirect FSW

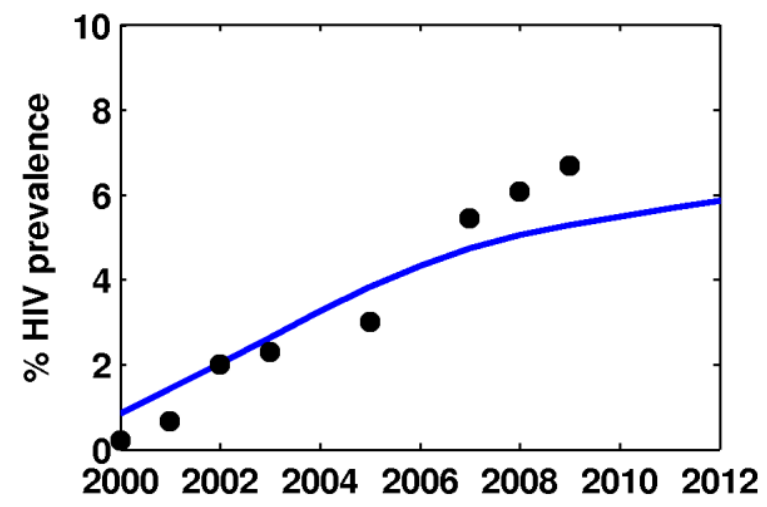




\section{Clients of FSW}

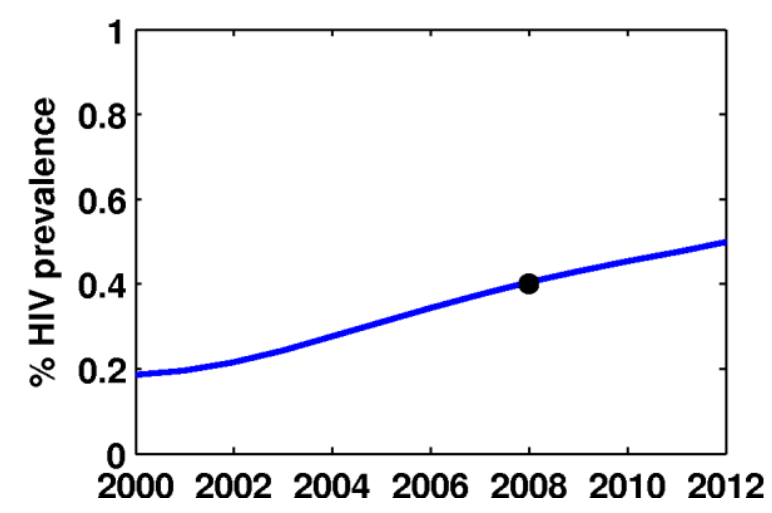

Overall prevalence in Papua

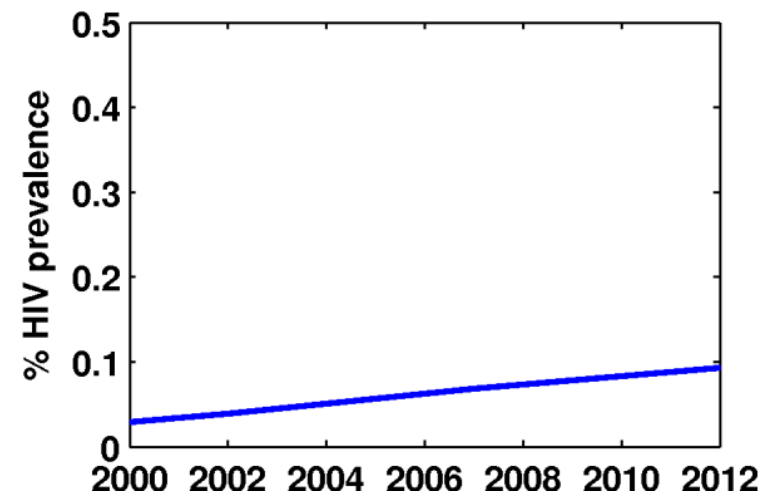




\section{Appendix 4: Relationships between HIV/AIDS Spending and Behavioral Parameters}

We used a generalized logistic function to relate each behavioral parameter affected by a HIV prevention program to the level of spending on that program. Using this function with assumed minimum and maximum values, we obtained logistic curve fits to available data on overall program spending and associated behavior. Minimum values correspond to calibrated parameter values for the year 2000, assumed to be prior to the introduction of any prevention programs. Calibrated parameter values from 2000 to 2010 are shown in the counterfactual scenarios in Appendix 5: Counterfactuals used in. We based the maximum/saturation value for the curves on knowledge from other settings.

As spending is not broken down by province or region, the logistic relationships for the five HIV prevention programs in non-Papua and Papua are based on the overall program spending at the national level. In addition, a large proportion of HIV spending has an unknown allocation or is unclassified. To account for this we reallocated the unknown/unallocated funding to the five program categories in proportion to $2009-2010$ budget allocation. This effectively rescales the spending horizontal axis on all the logistic curves shown below.

In the plots black discs represent estimates based on available data, the blue line is the fitted curve and the red disc shows the level of spending in 2010. It is important to note that each plot shows the level of behavior in relation to our reallocated estimate of overall national spending on that program.

\section{Non-Papua}

\section{Relationships between FSW behavioral parameters and spending on programs prioritizing FSW and clients - Non-Papua}

Condom use with regular partners

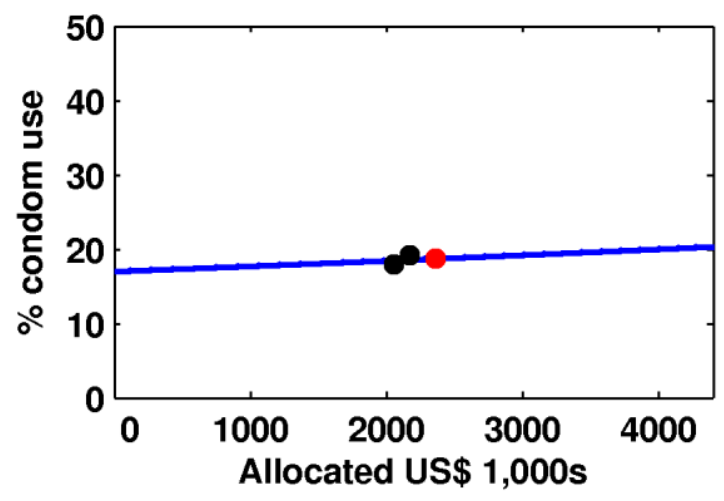

Condom use with casual partners

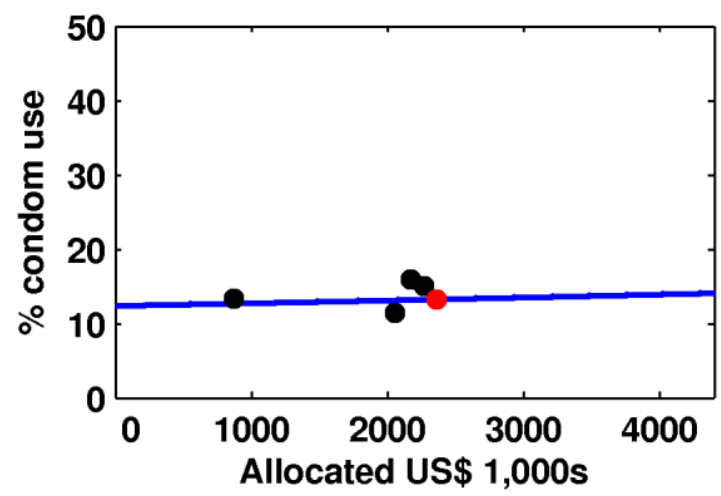


Condom use with clients: direct

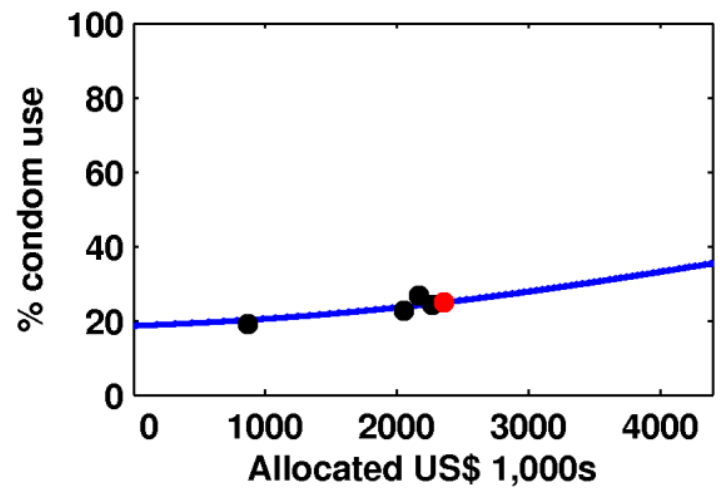

Number of clients: direct FSW
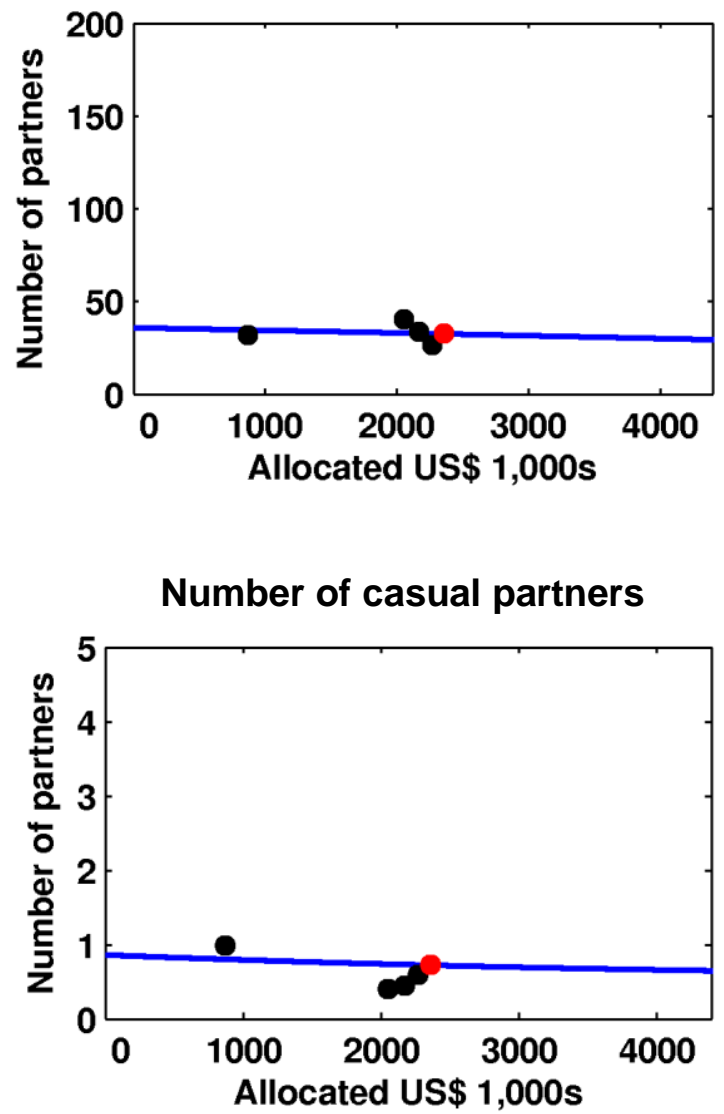

Condom use with clients: indirect

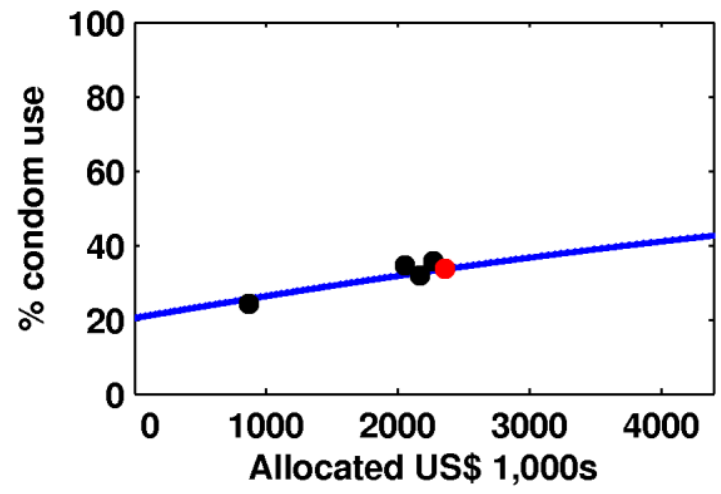

Number of clients: indirect FSW

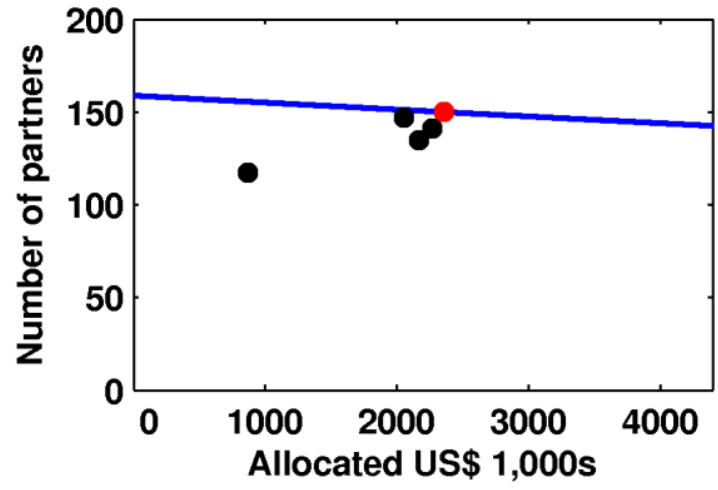

Number of regular partners

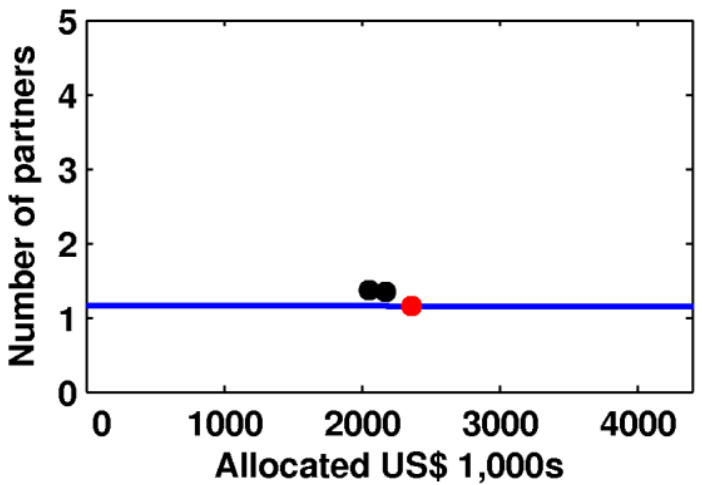


HIV testing

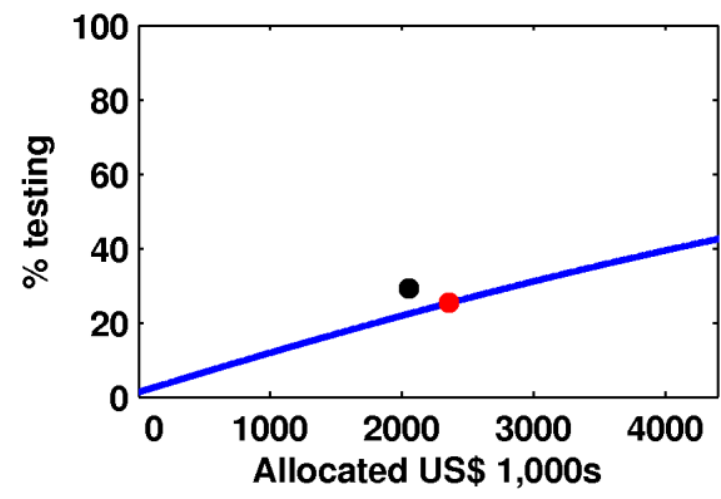

Relationships between the behavioral parameters of clients and spending on programs prioritizing FSW and clients - Non-Papua

Condom use with regular partners

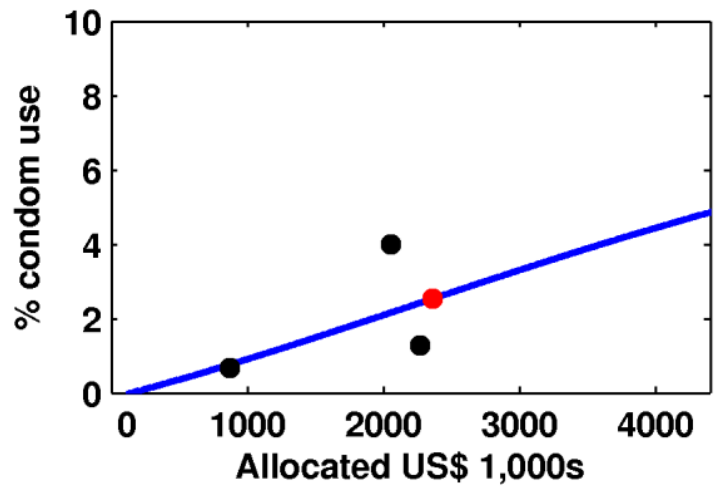

Condom use with casual partners

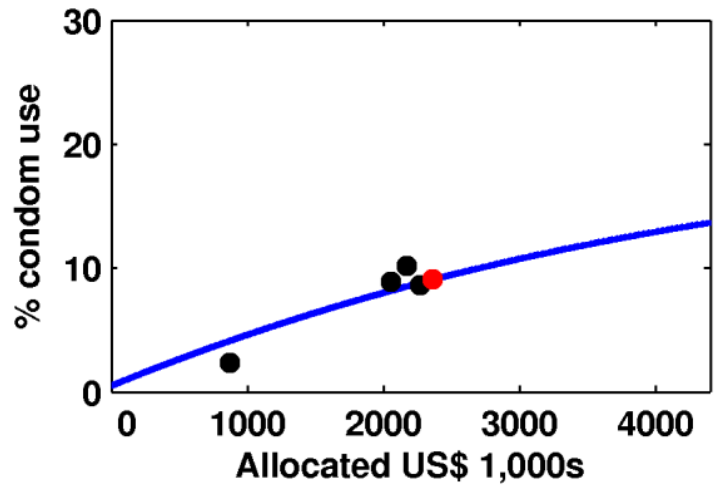

Condom use with FSW

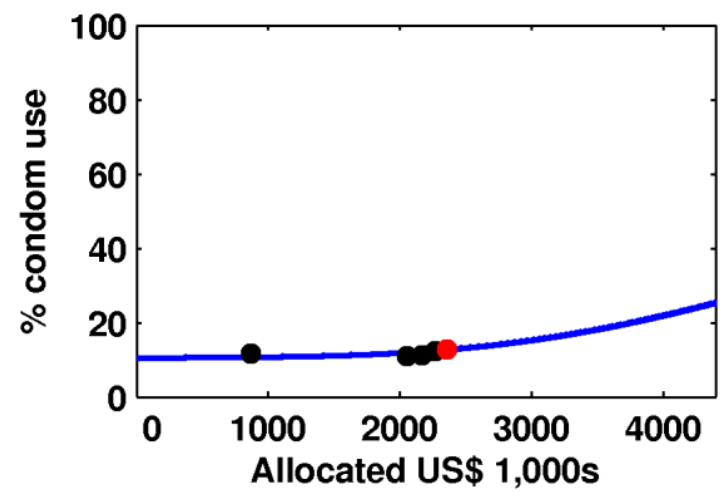

Number of regular partners

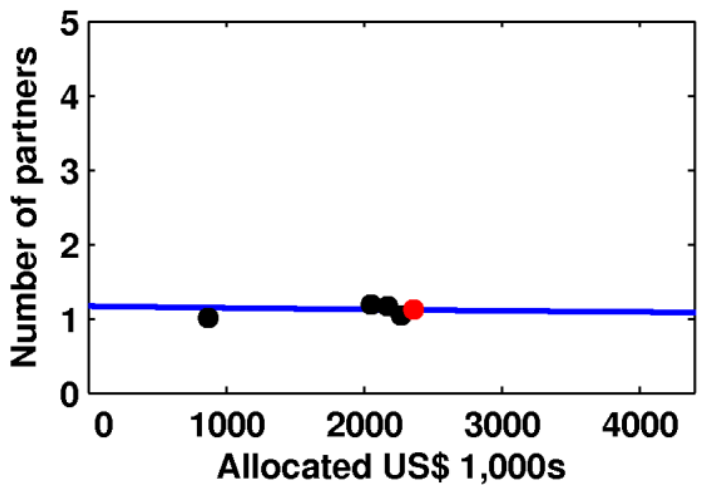



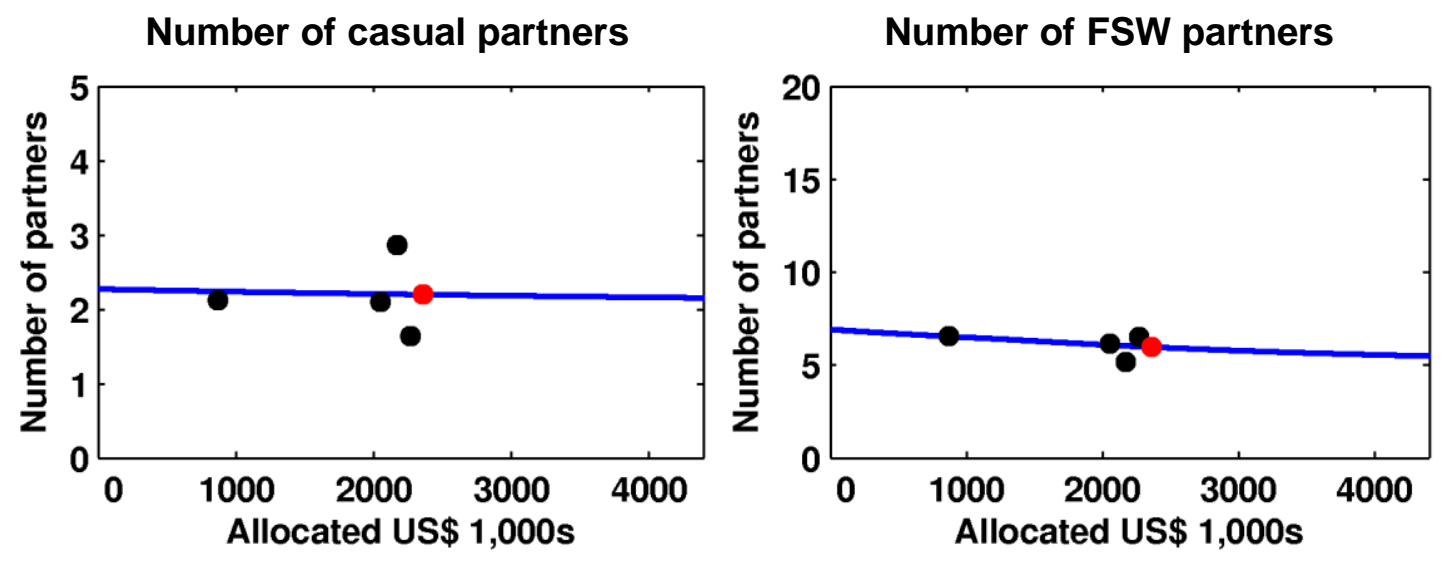

HIV testing

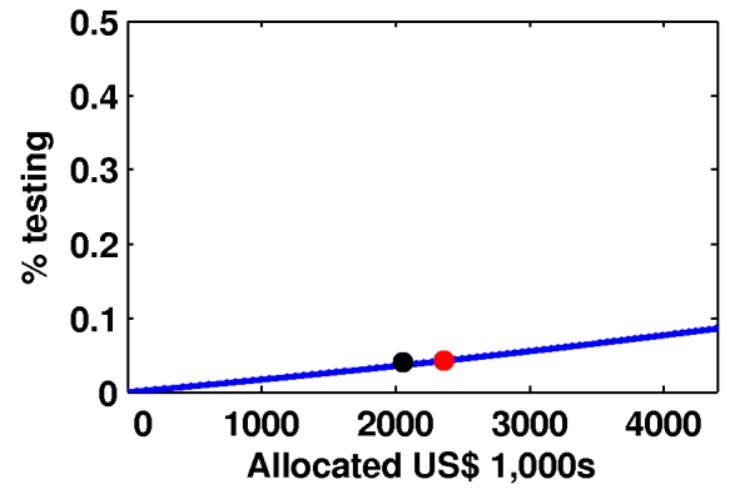

Relationships between the behavioral parameters of MSM and spending on programs prioritizing MSM and Waria - Non-Papua
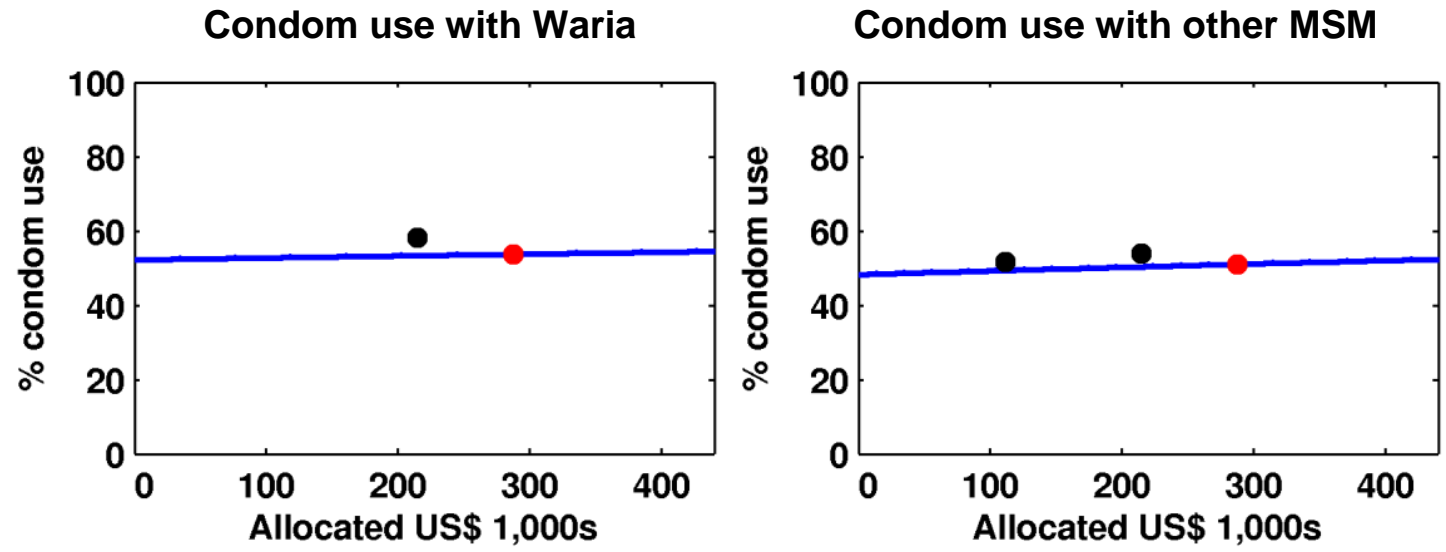

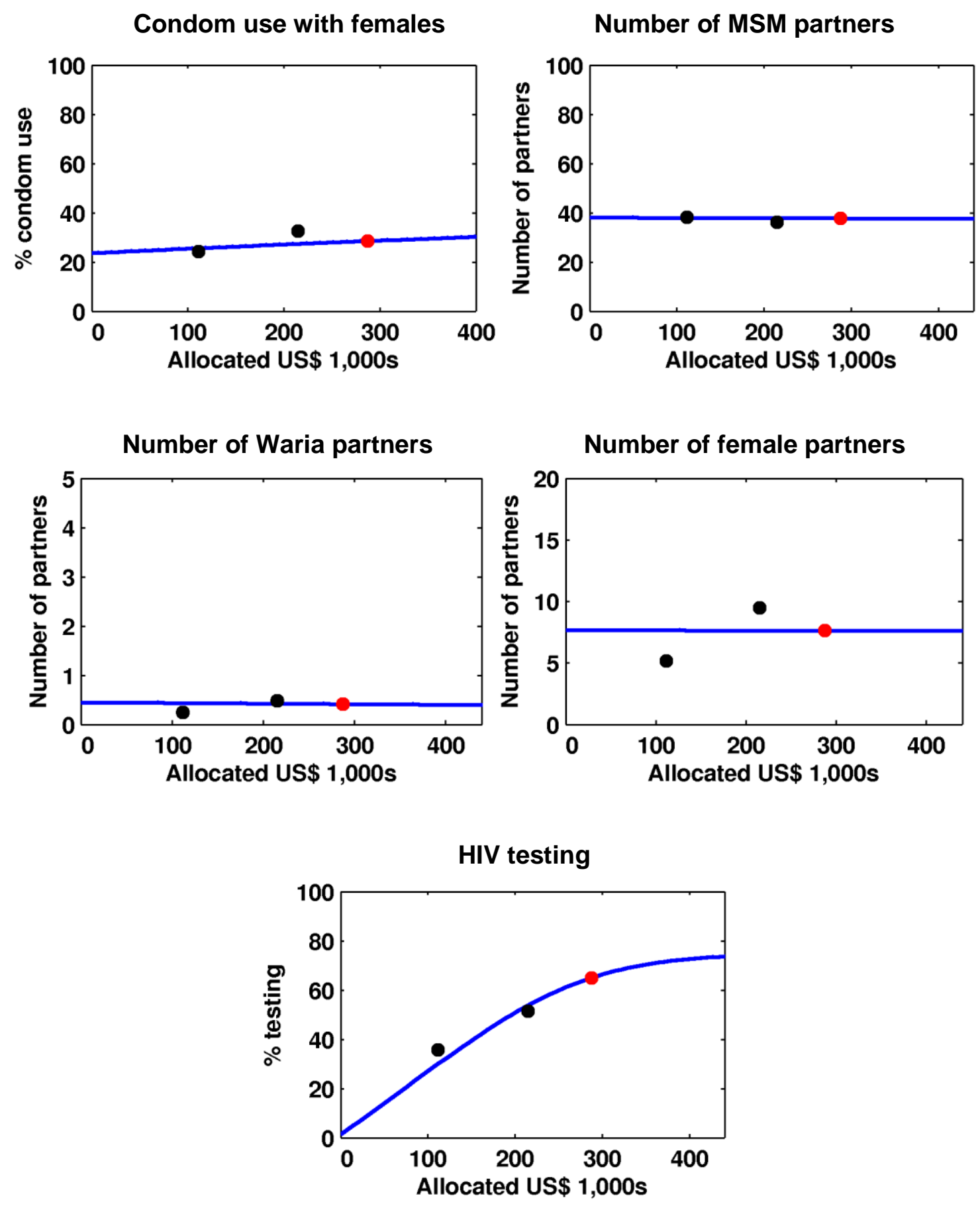
Relationships between the behavioral parameters of Waria and spending on programs prioritizing MSM and Waria - Non-Papua

Condom use with casual partners

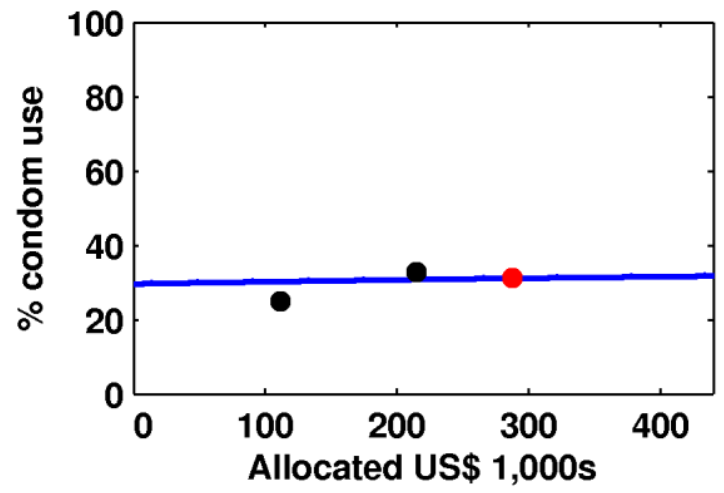

Number of casual partners

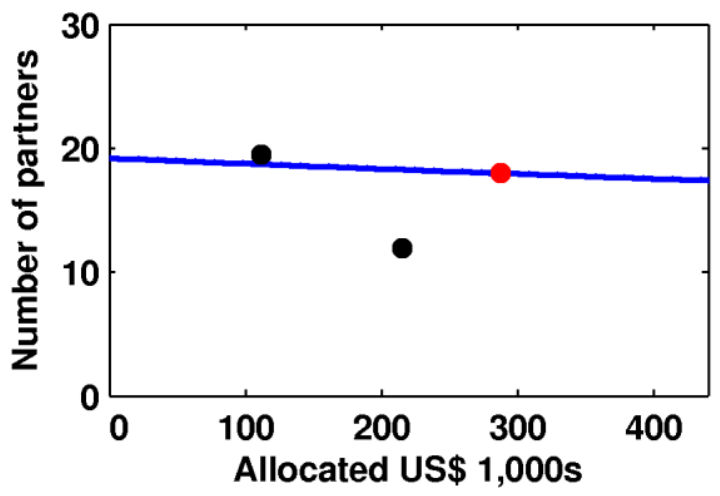

Condom use with commercial partners

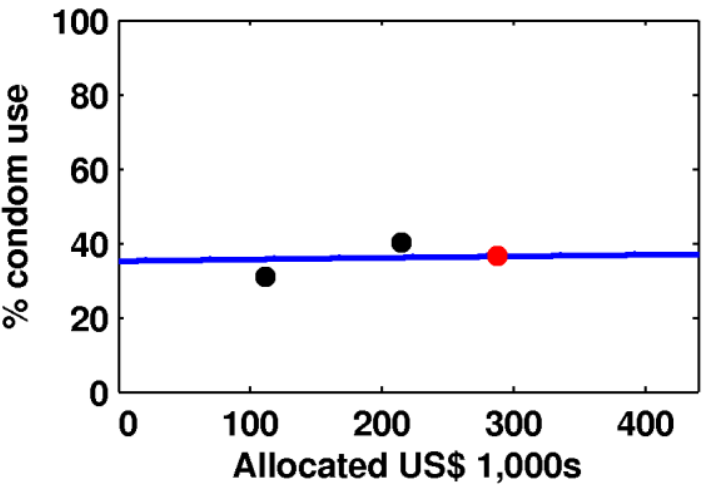

Number of commercial partners

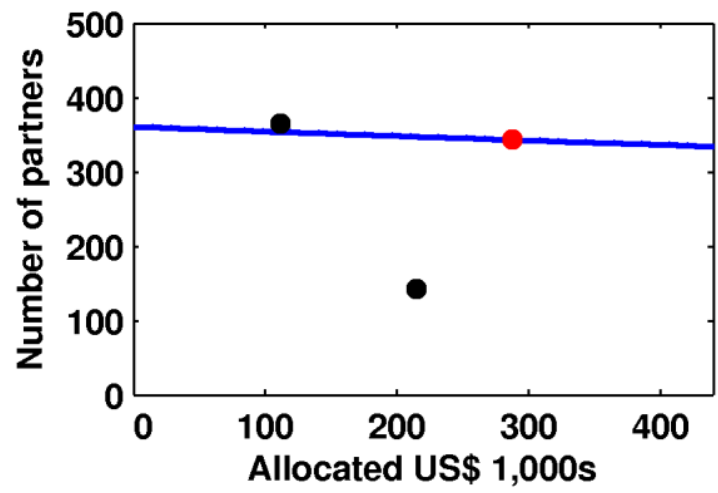

HIV testing

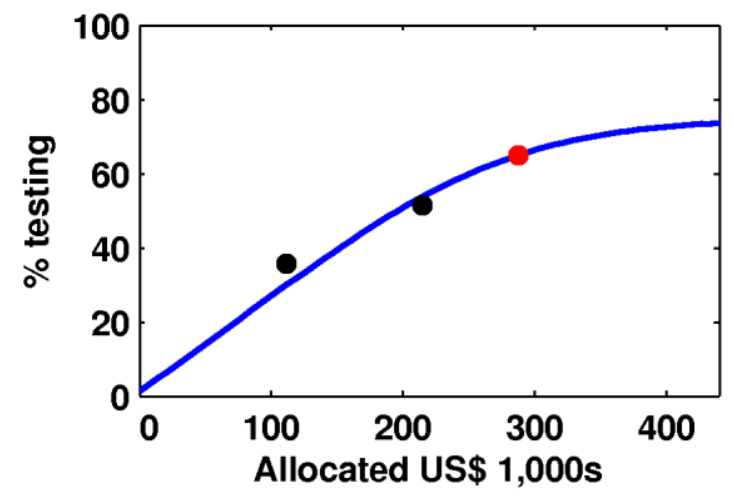


Relationships between the behavioral parameters of IDU and spending on NSP programs - Non-Papua

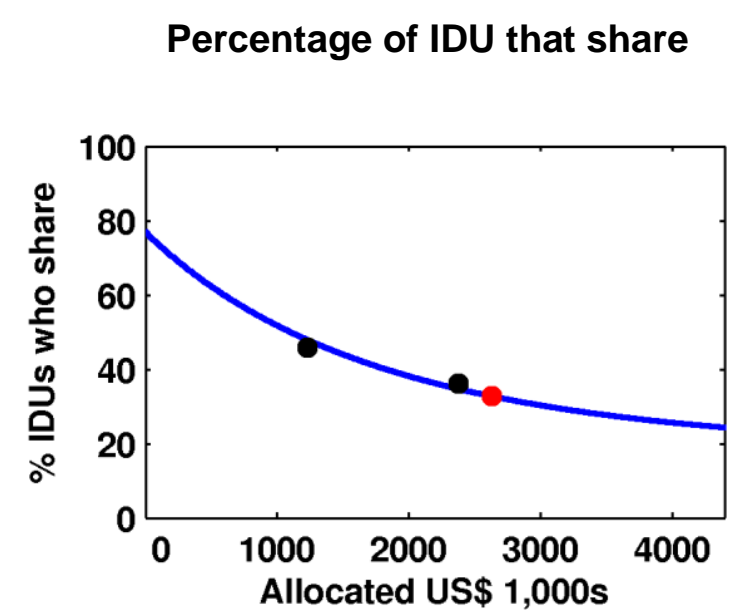

Condom use with regular partners

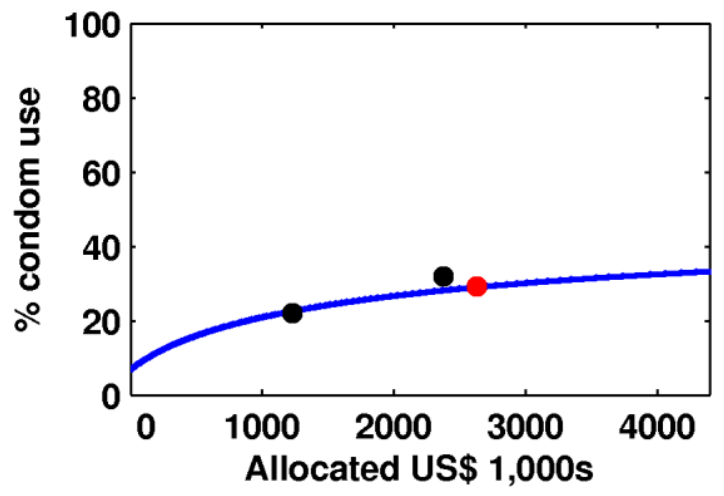

For IDU who share the percentage of injections shared

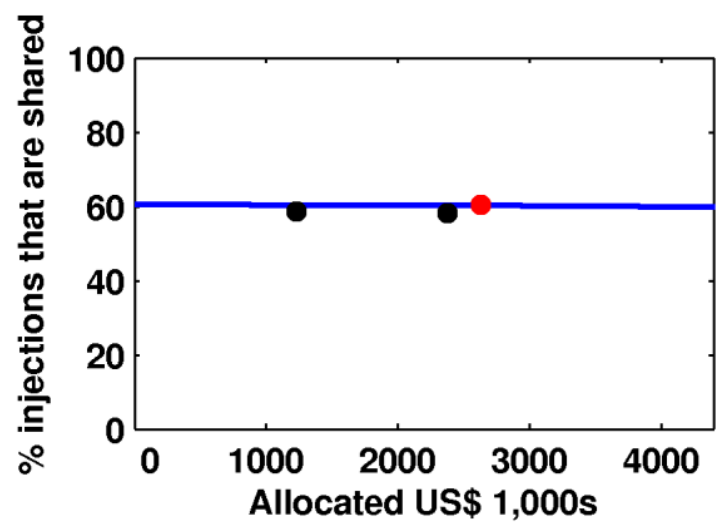

Condom use with casual partners

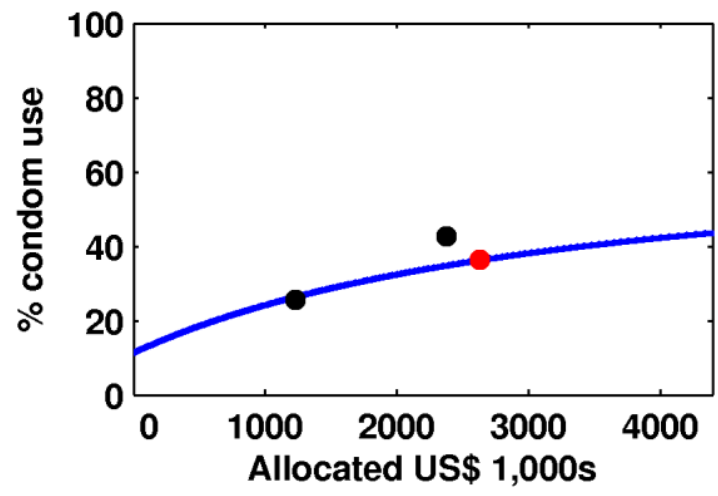


Condom use with commercial partners

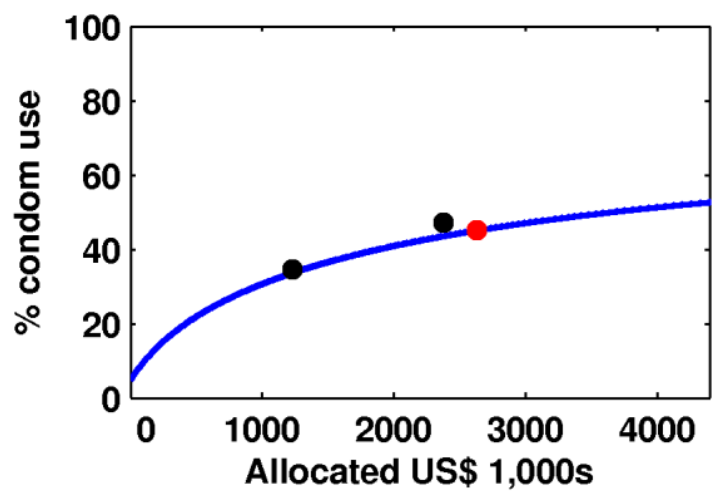

Number of casual partners

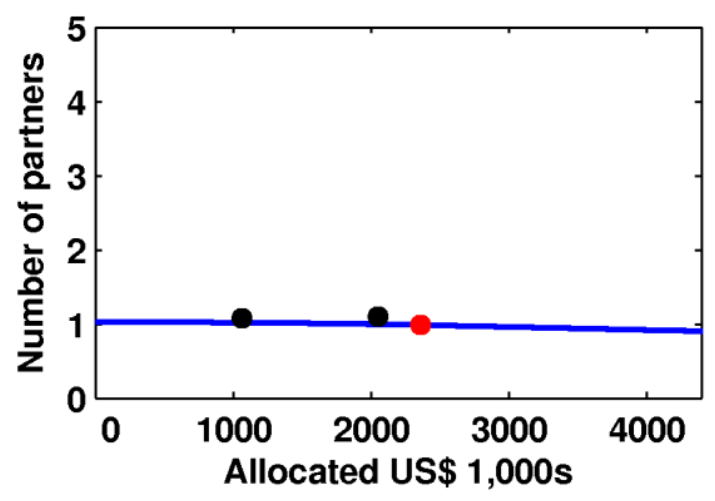

Number of regular partners

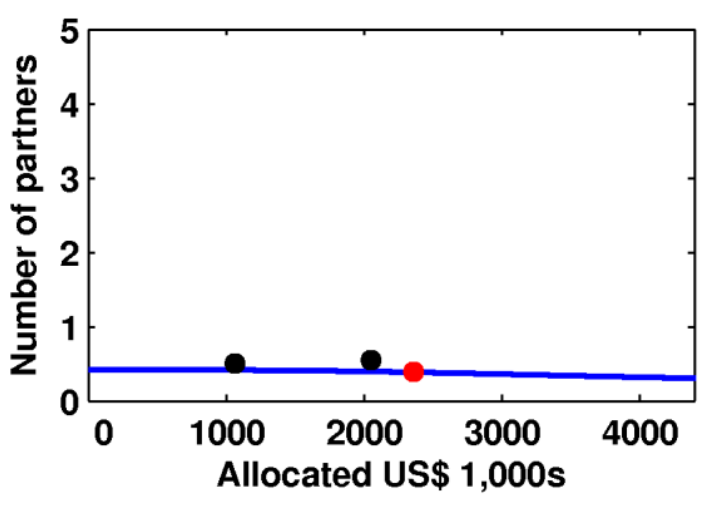

Number of commercial partners

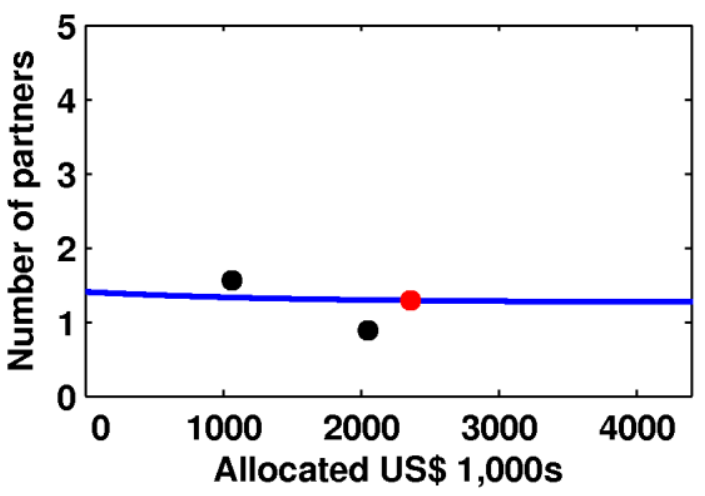

HIV testing

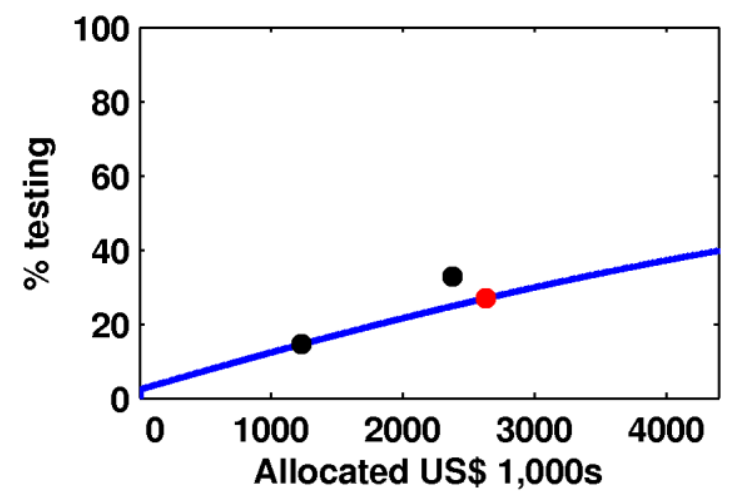


Relationships between the behavioral parameters of IDU and spending on MMT programs - Non-Papua

Percentage of IDU on MMT

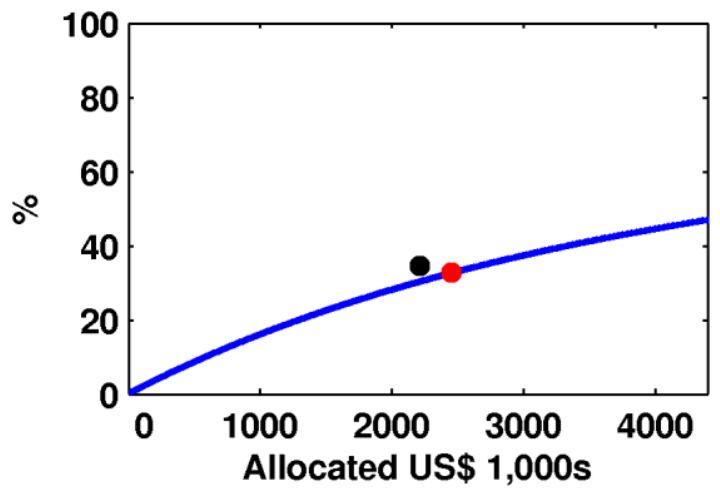

Number of injections per year

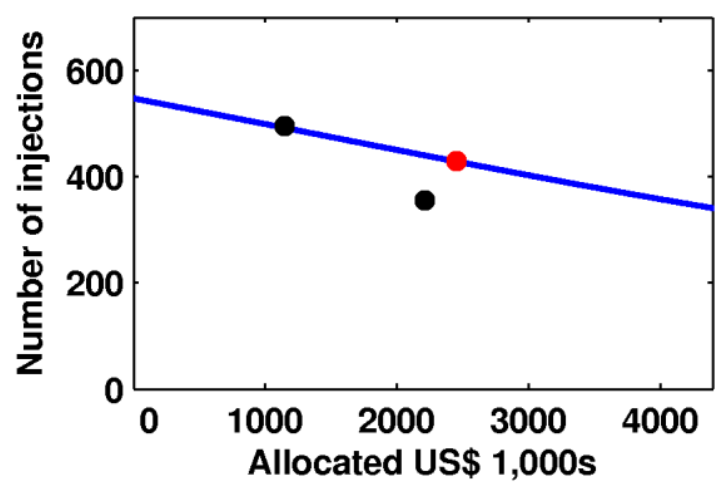

Relationships between low risk male and female behavioral parameters and spending on programs for the general population - Non-Papua

Condom use with regular partners: males

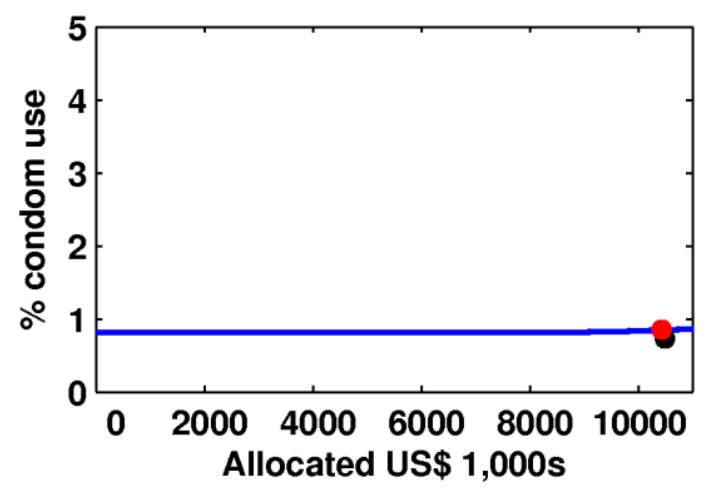

Condom use with regular partners: females

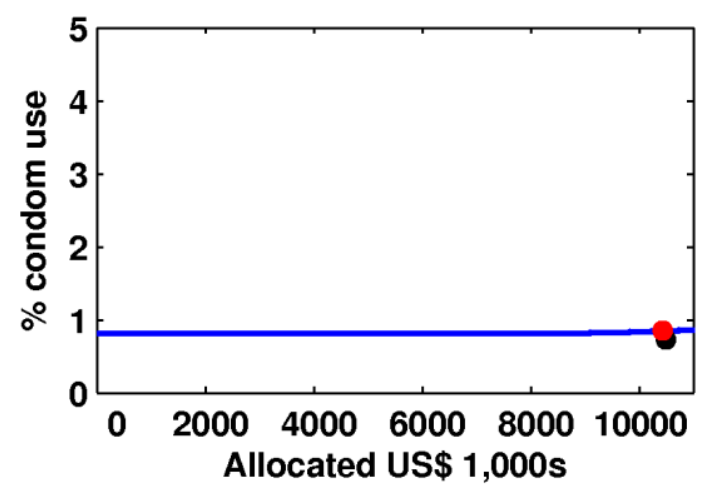


Condom use with casual

partners: males

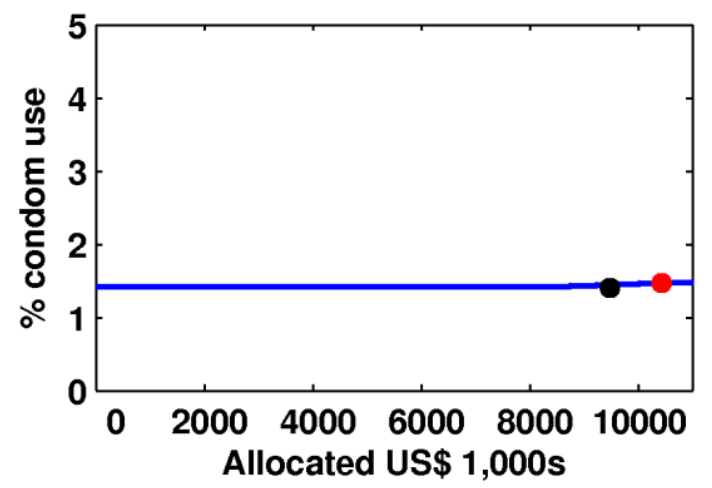

Number of regular partners: males

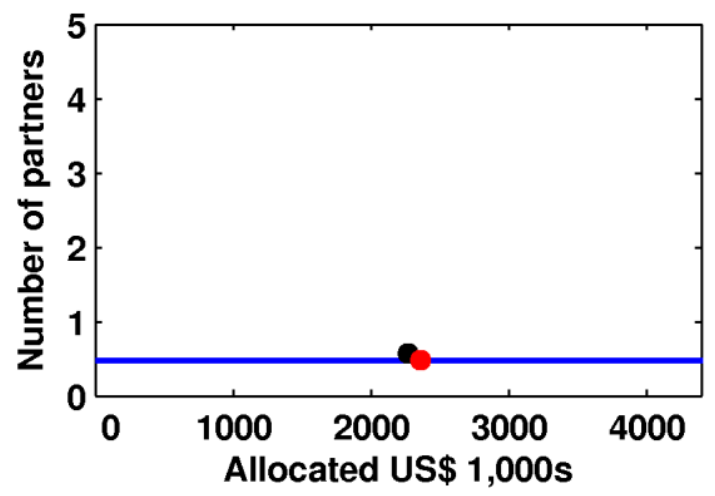

Number of casual partners: males

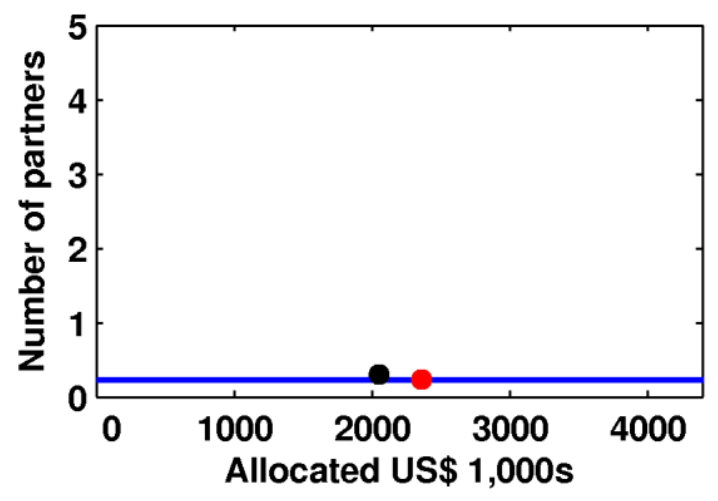

Condom use with casual

partners: females

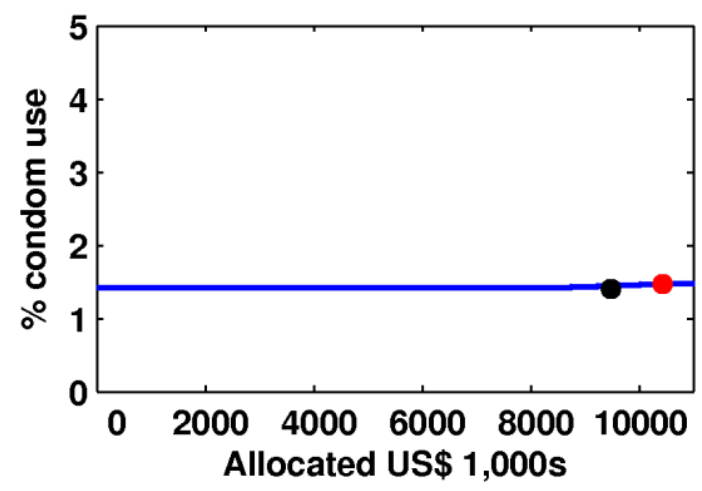

Number of regular partners: females

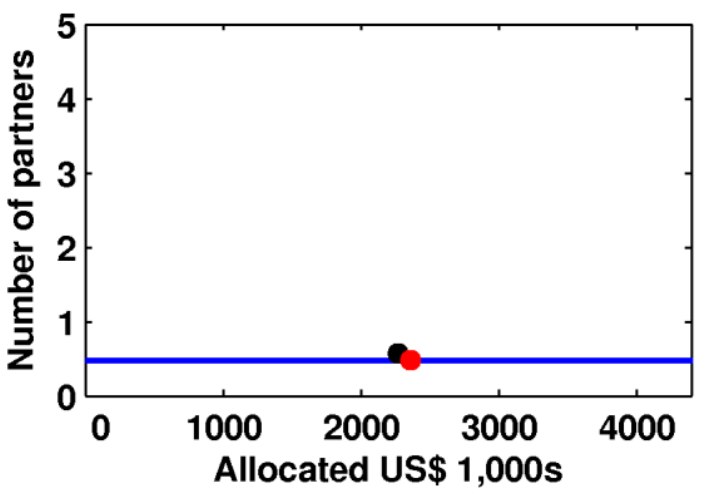

Number of casual partners: females

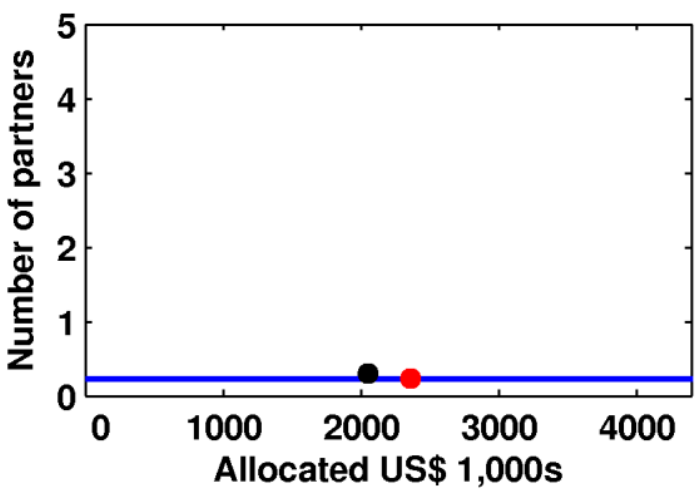


HIV testing

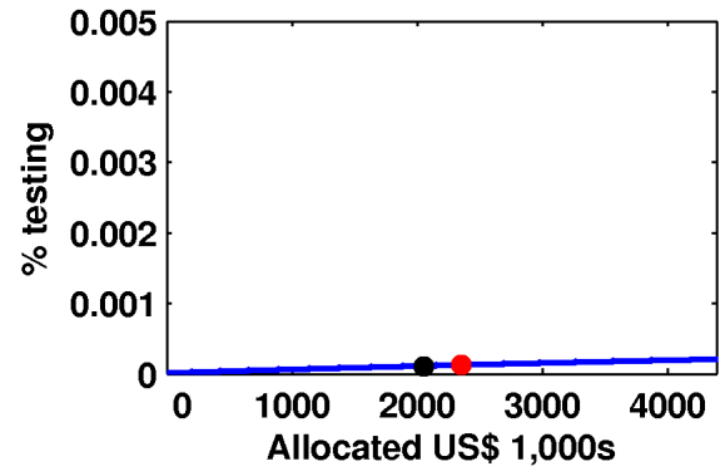

Papua

Relationships between FSW behavioral parameters and spending on programs prioritizing FSW and clients - Papua

Condom use with casual partners Condom use with regular partners
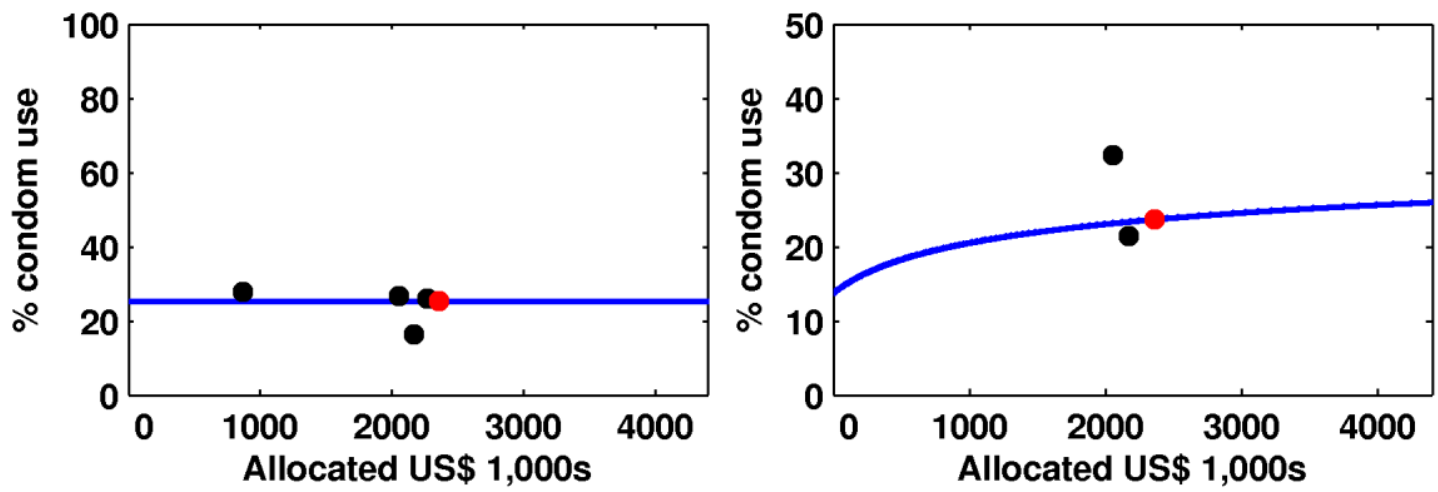
Condom use with clients: direct

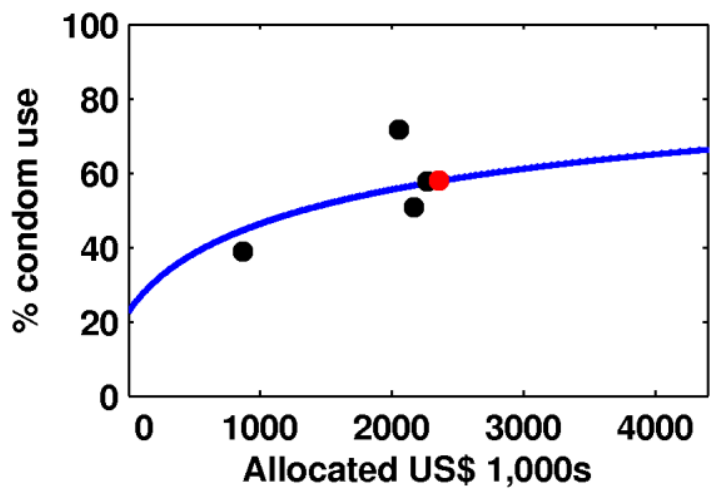

Number of clients: indirect

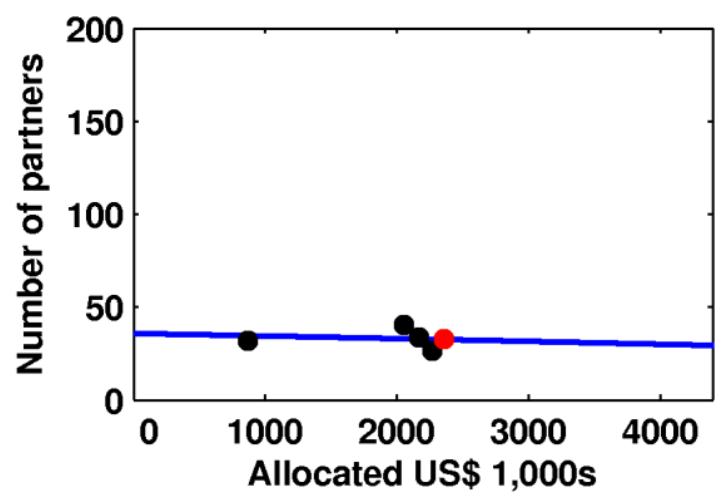

Number of casual partners

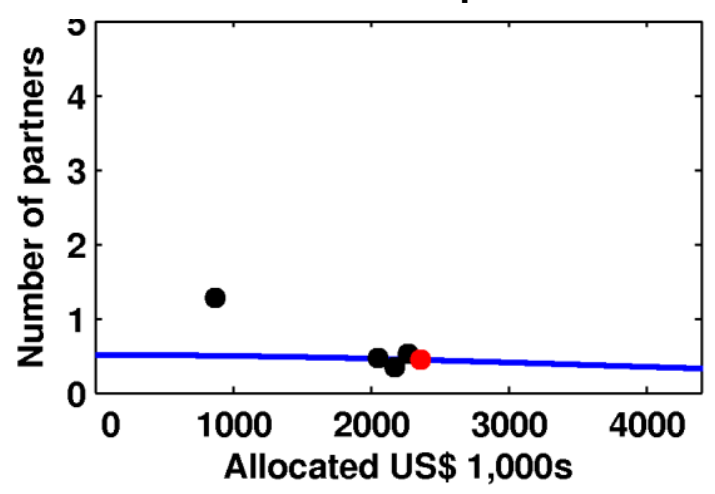

Condom use with clients: indirect

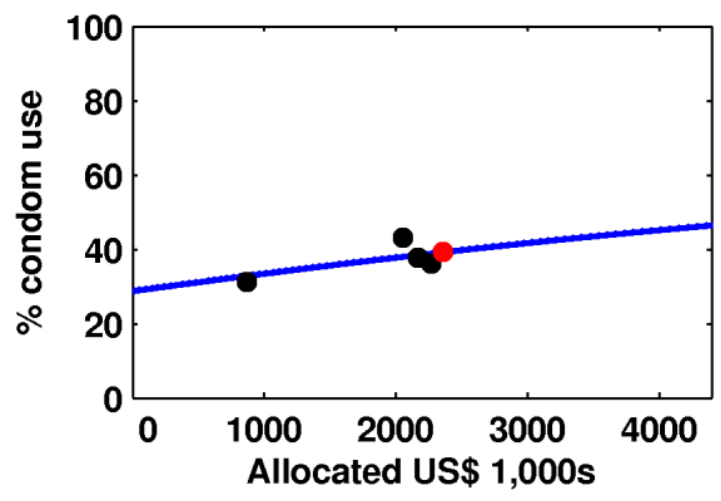

Number of clients: direct

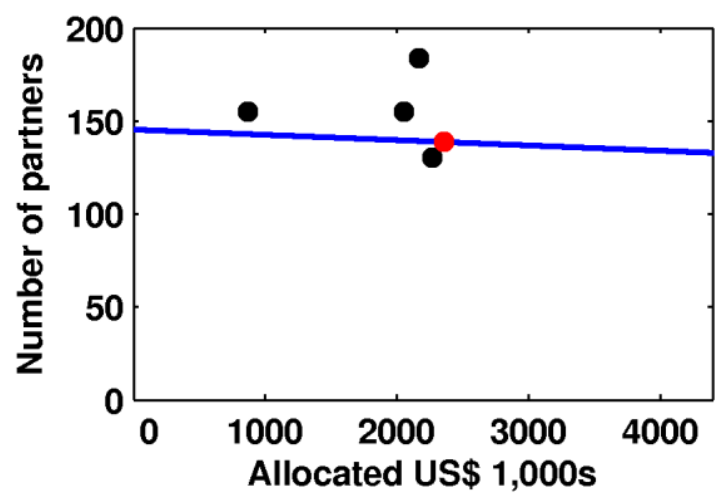

Number of regular partners

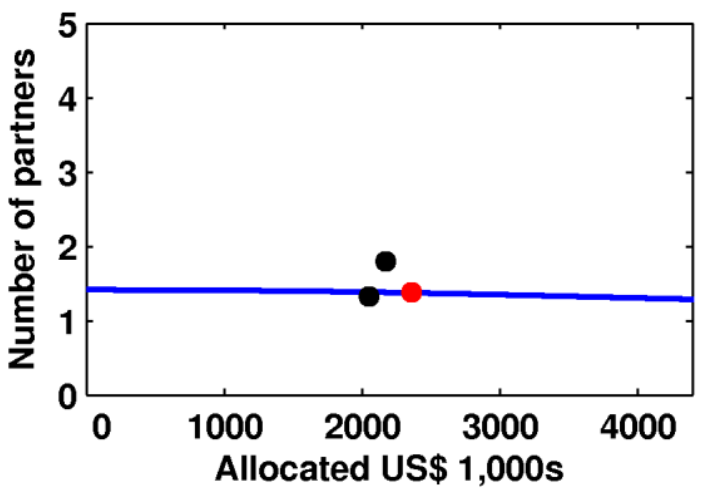


HIV testing

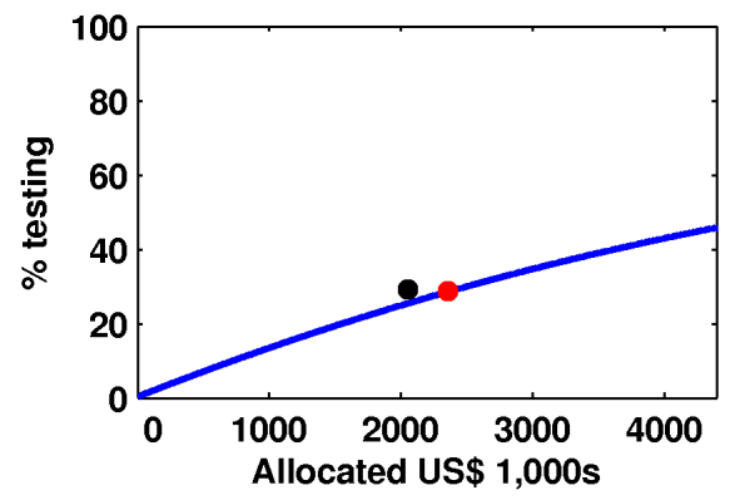

Relationships between the behavioral parameters of clients and spending on programs prioritizing FSW and clients - Papua

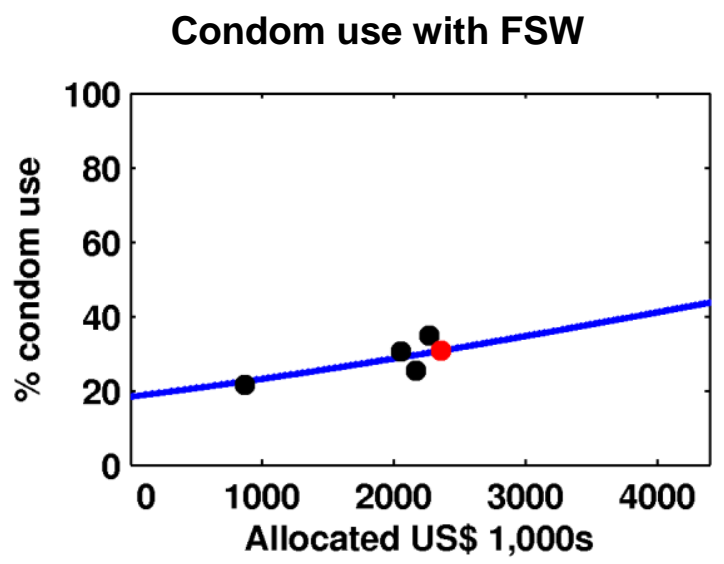

Condom use with casual partners

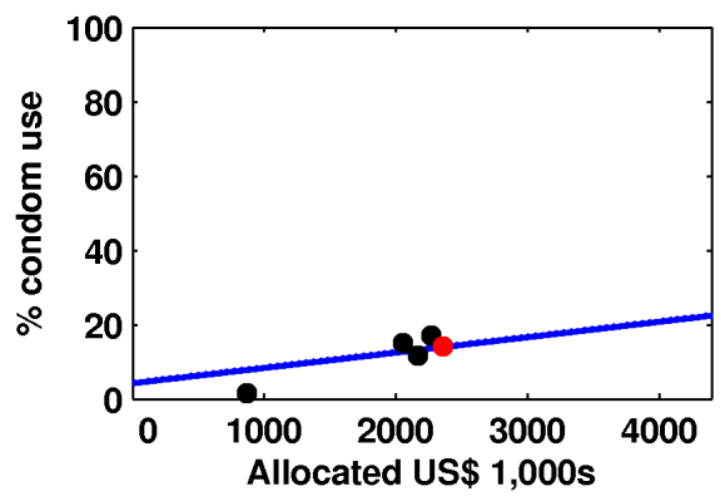

Condom use with regular partners Number of FSW partners
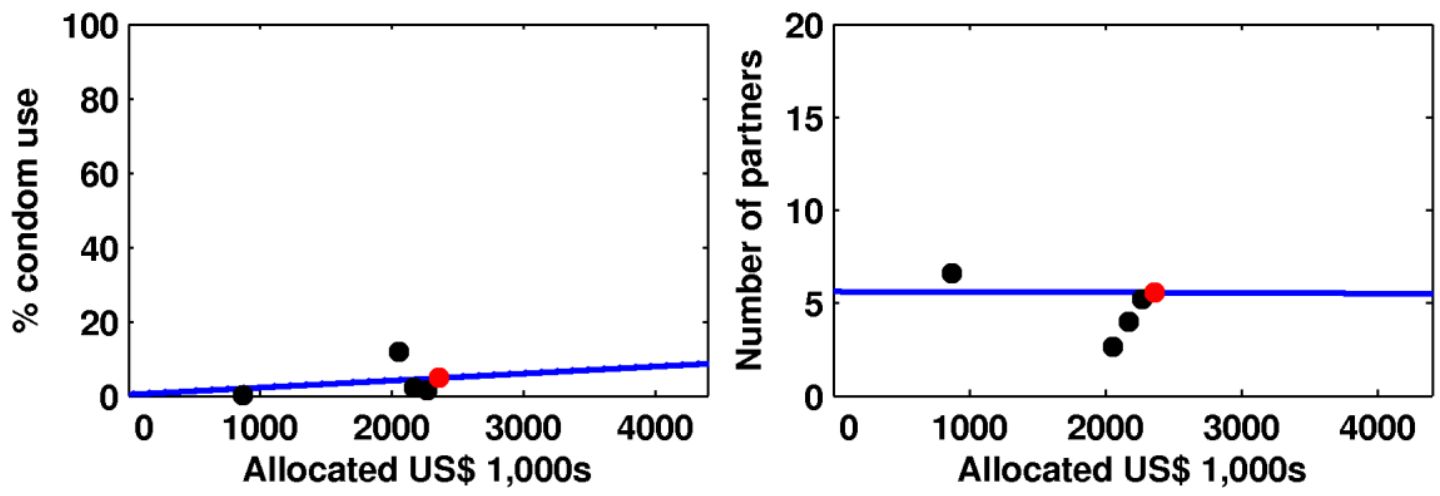

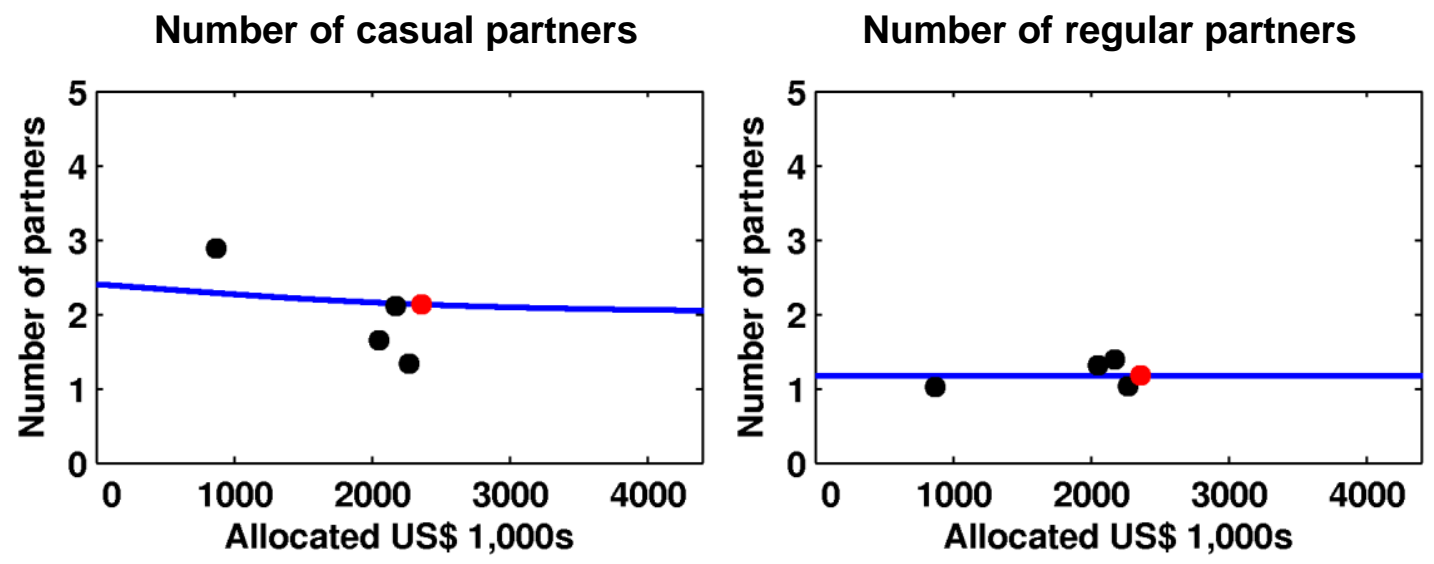

HIV testing

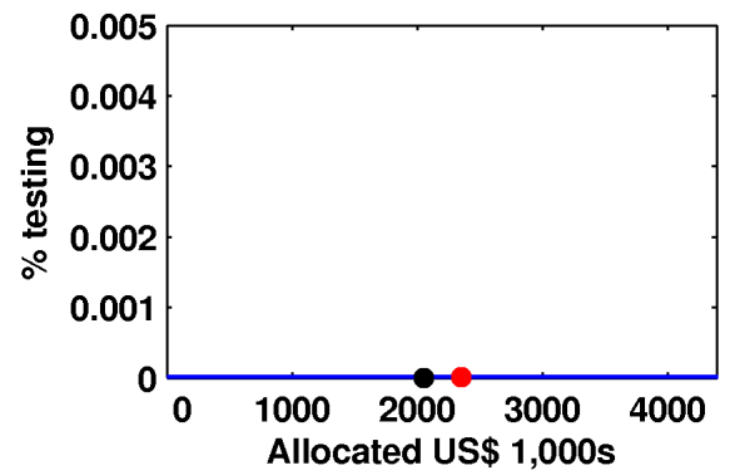


Condom use with casual

partners: males

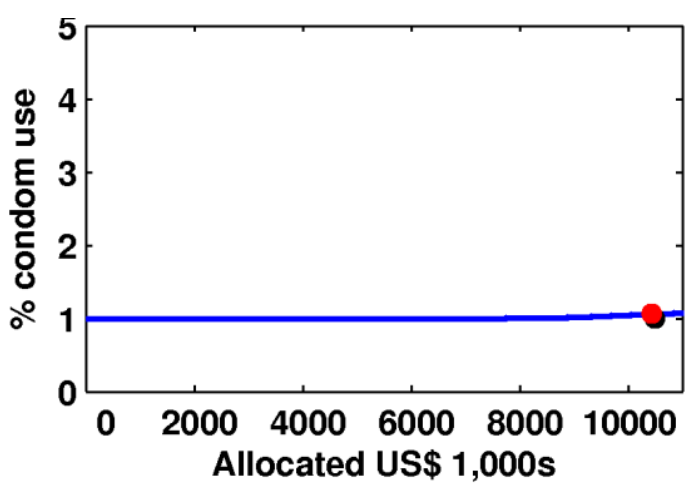

Condom use with regular partners: males

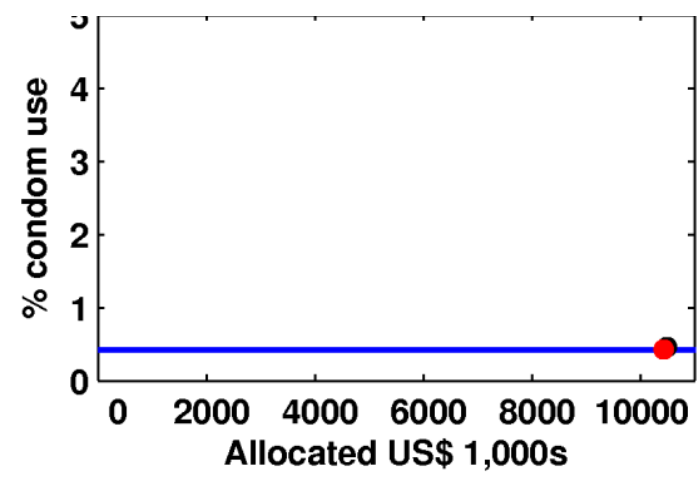

Number of casual partners: males

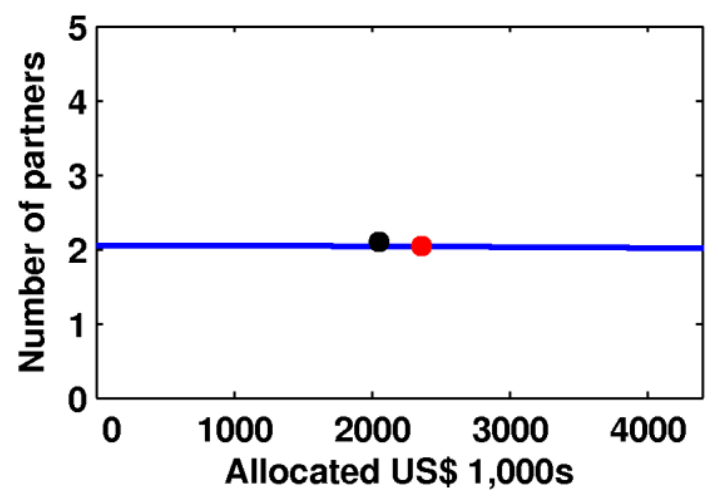

Condom use with casual

partners: females

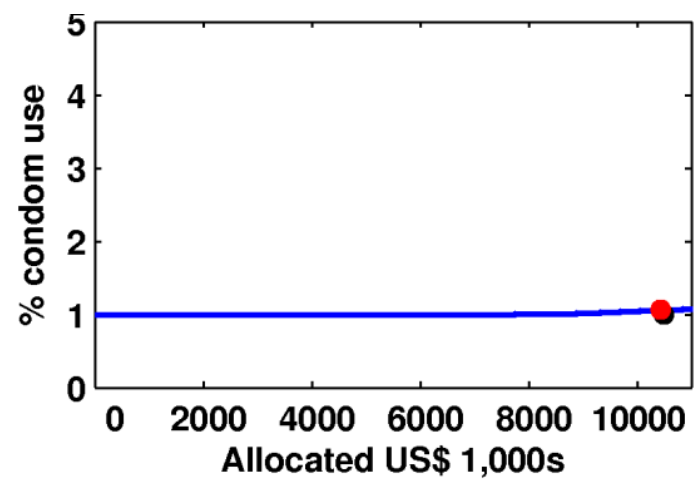

Condom use with regular partners: females

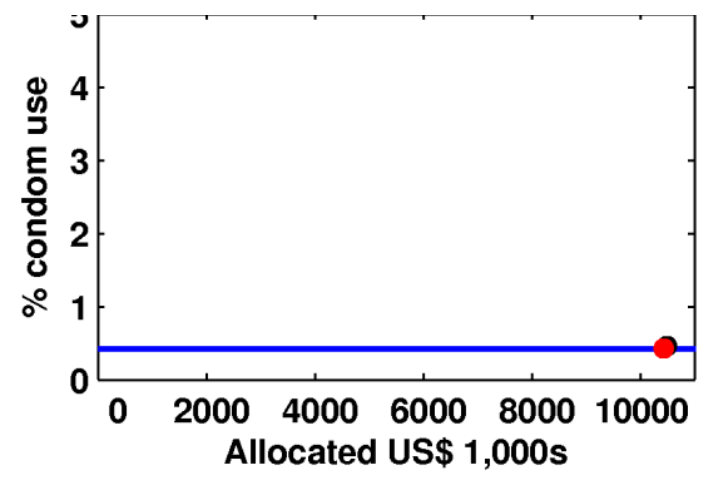

Number of casual partners: females

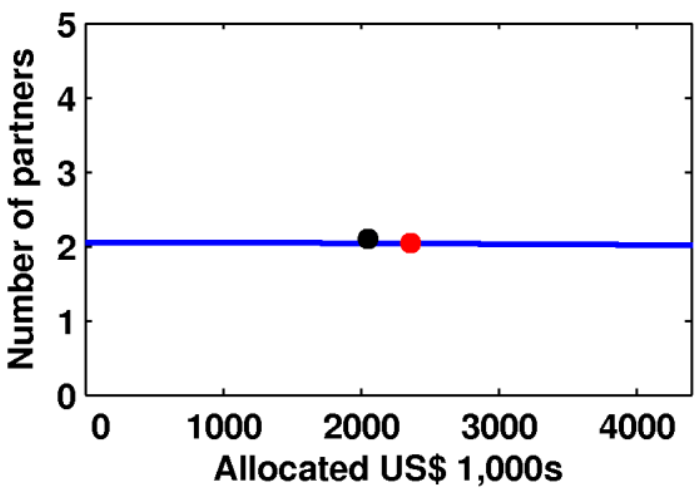


Number of regular partners: males

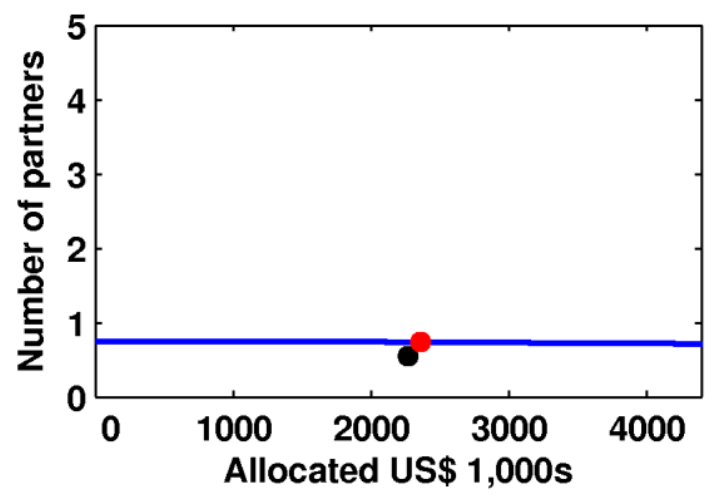

Number of regular partners: females

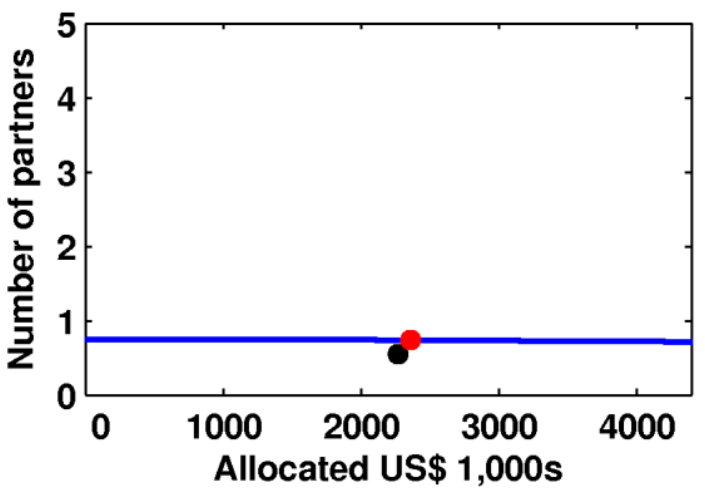

HIV testing

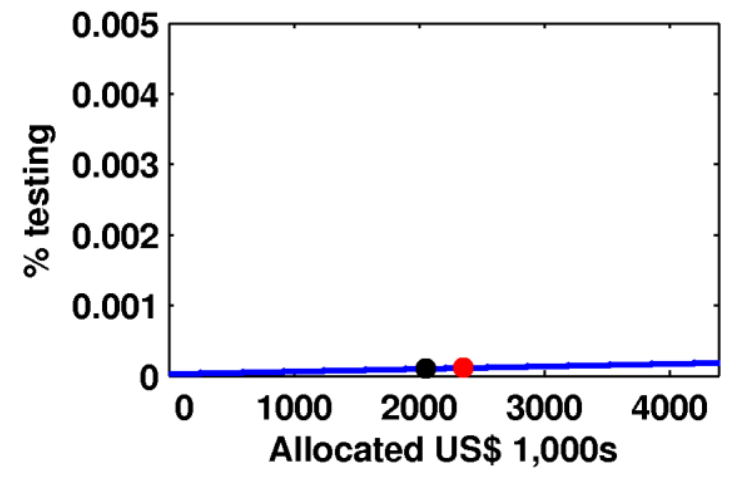




\section{Appendix 5: Counterfactuals used in analyses}

To determine the cost-effectiveness of HIV program funding in Indonesia we simulated counterfactual scenarios for the 2000 to 2010 period using HIM. These scenarios were based on the assumed effect of the removal of specific programs. For each prioritized population, we developed two counterfactual scenarios for the behavioral parameters affected by prevention programs prioritizing that population. The parameters for the other populations remained at their calibrated values. One counterfactual assumed all changes in behavior since 2000 was due to the introduction of the prevention programs. The other counterfactual assumed that $50 \%$ of the annual change in parameter values from 2000 to 2010 was due to the program with the remaining $50 \%$ of change occurring naturally or due to indirect effects from programs prioritizing other populations.

All counterfactual scenarios are plotted below. In these plots, the black discs represent available data and the blue line is the fitted values obtained during calibration using HIM. The blue circle marking the $y$-intercept is the 2000 model value for all the parameters determined during model calibration. We assume these values represent behavior prior to the introduction of HIV prevention programs. Red lines represent the two counterfactual scenarios with cross-hatching representing the potential range for changes due to the presence of prevention programs.

Cost-effectiveness analyses were carried out to compare outcomes (QALYs gained) and costs between each counterfactual scenario against the current conditions.

The unit costs shown in Table 9 were used to estimate the total amount spent on health care of individuals with HIV in each year.

The incremental cost-effectiveness ratio (ICER), expressed as cost per QALY gained, was computed as the difference between the total healthcare costs in current conditions and without the intervention (for the lifetime of the program) divided by the difference between the total QALYs in current conditions and without the intervention. Costs were discounted by an annual rate of 3\% (and 10\%). ICERs were computed for two time horizons: from 2000-2010 and 2000Lifetime, which is assumed to be 2000-2100.

Total costs for implementation of the program were estimated from NASA data and total costs indicated in UNGASS reports. 


\section{Non-Papua}

Counterfactuals for programs for FSW and their clients

Female sex workers

Condom use with regular partners

Condom use with casual partners

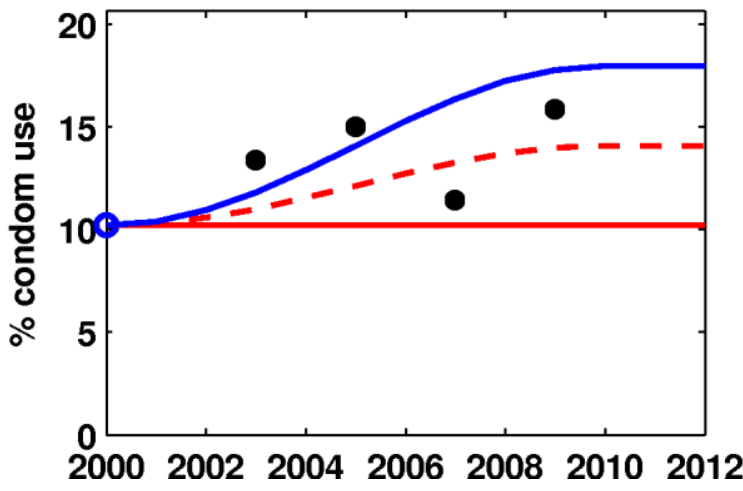

Condom use with clients: direct

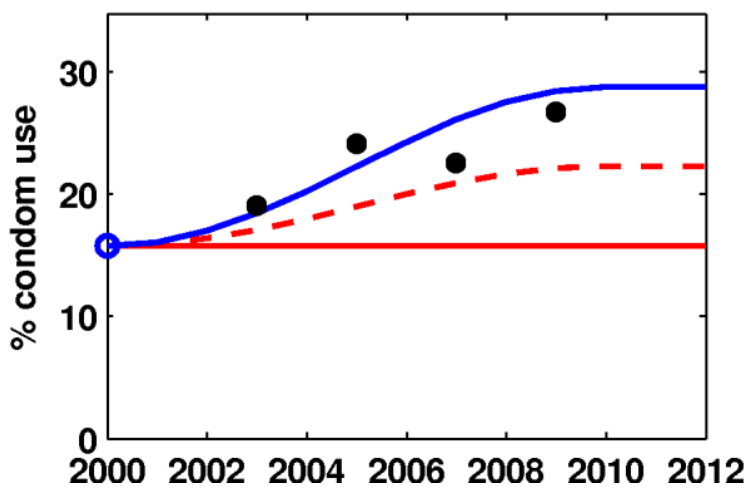

Number of casual partners

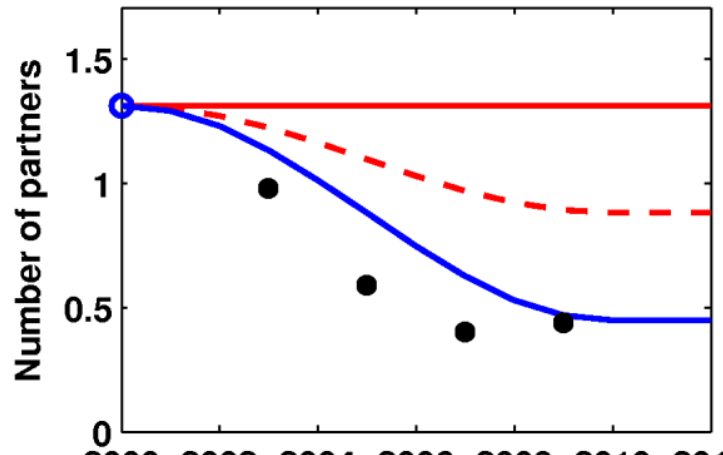

2000200220042006200820102012
Number of casual partners

Condom use with regular partners

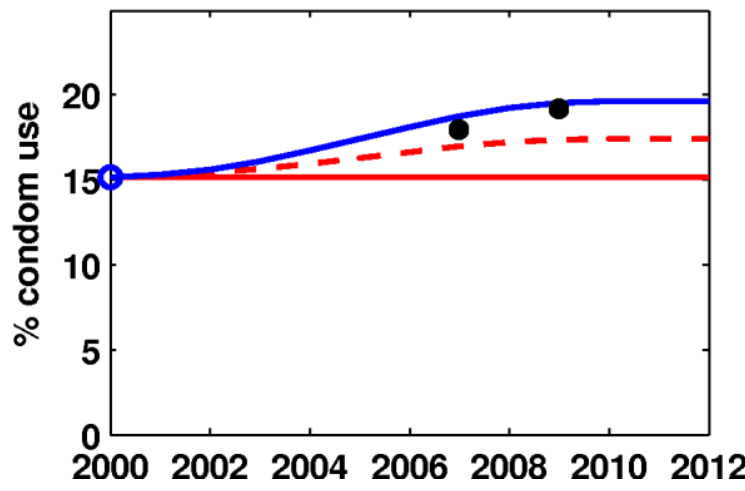

Condom use with clients: indirect

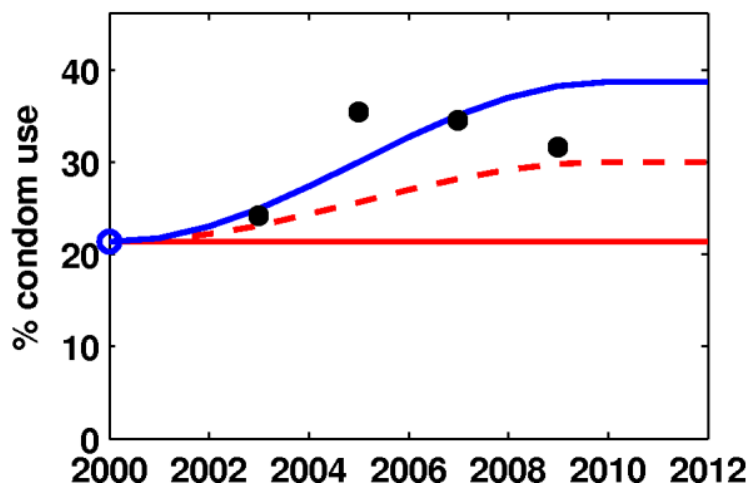

Number of regular partners

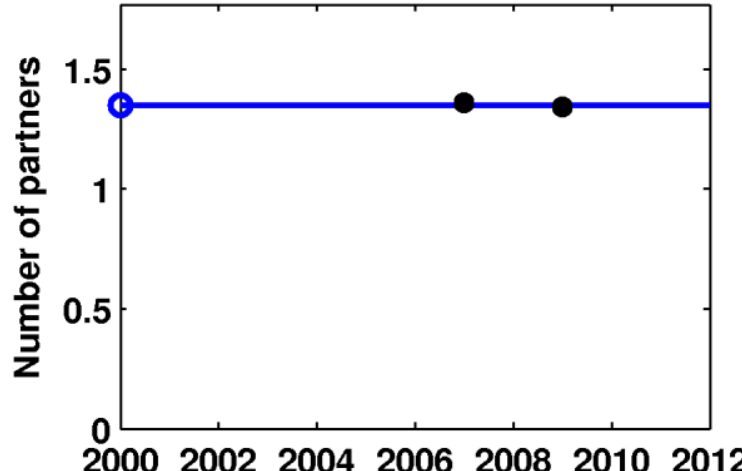


Number of clients: direct

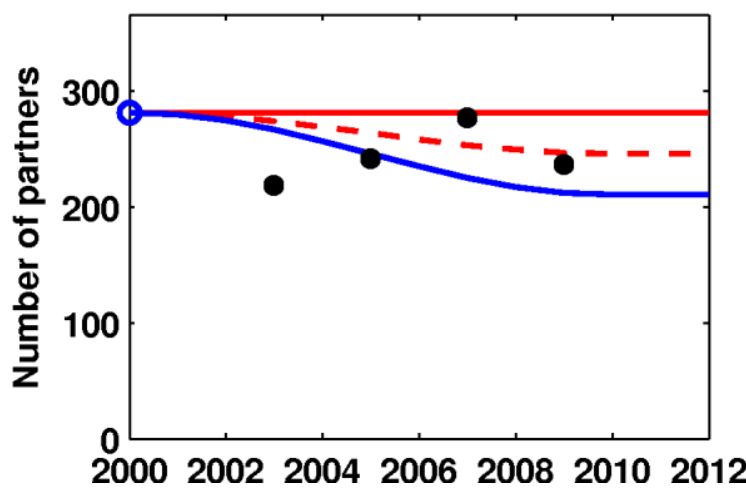

Number of clients: indirect

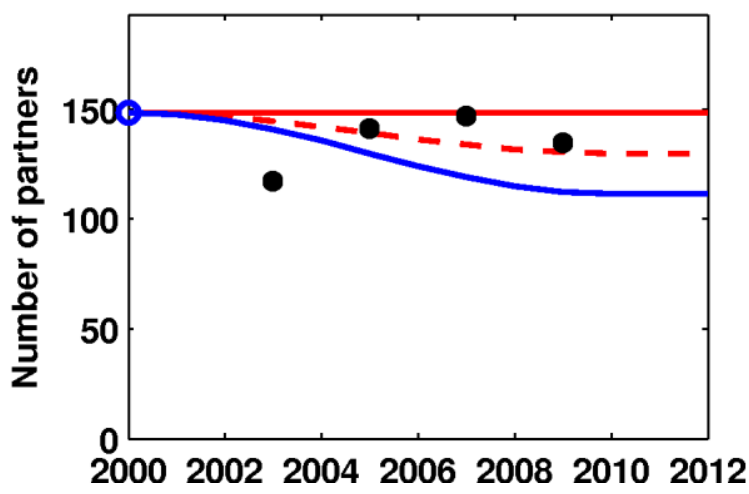

HIV Testing

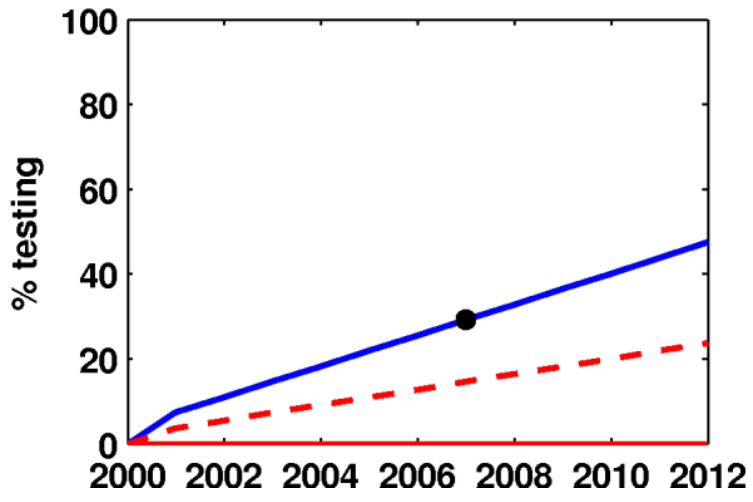

Clients of FSW

Condom use with casual partners

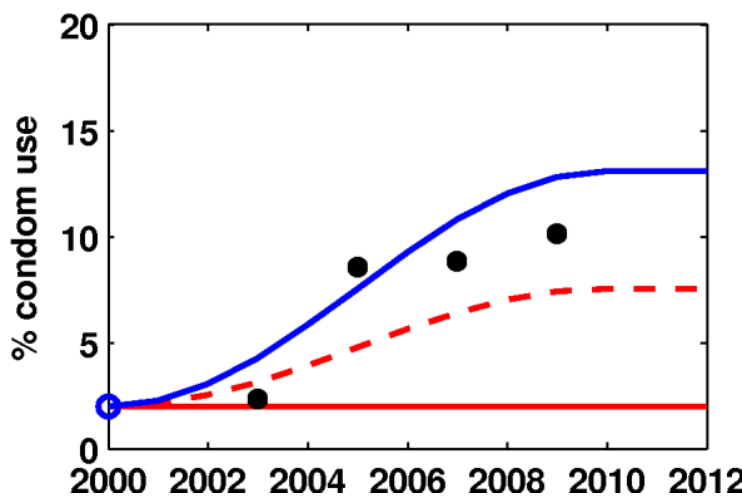

Condom use with FSW

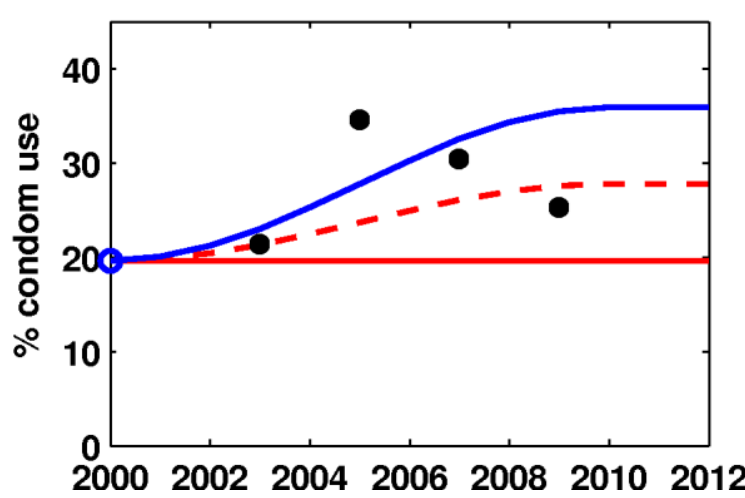


Condom use with regular partners

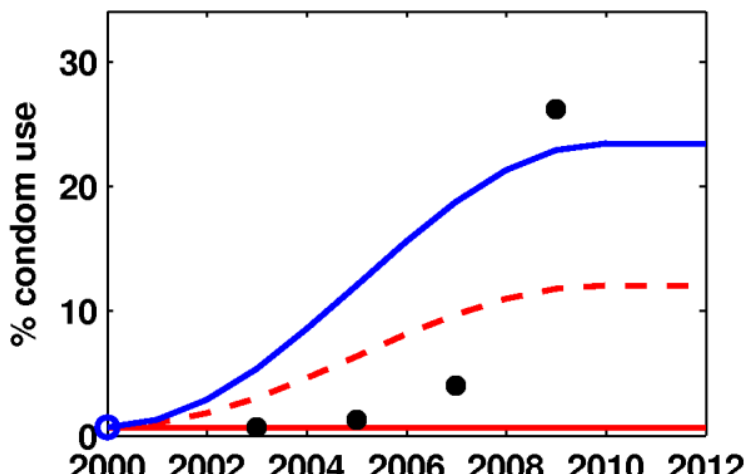

Number of regular partners

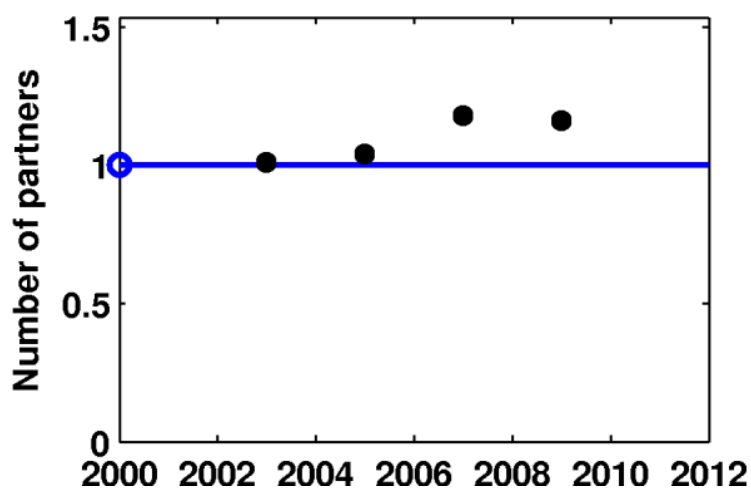

Number of casual partners
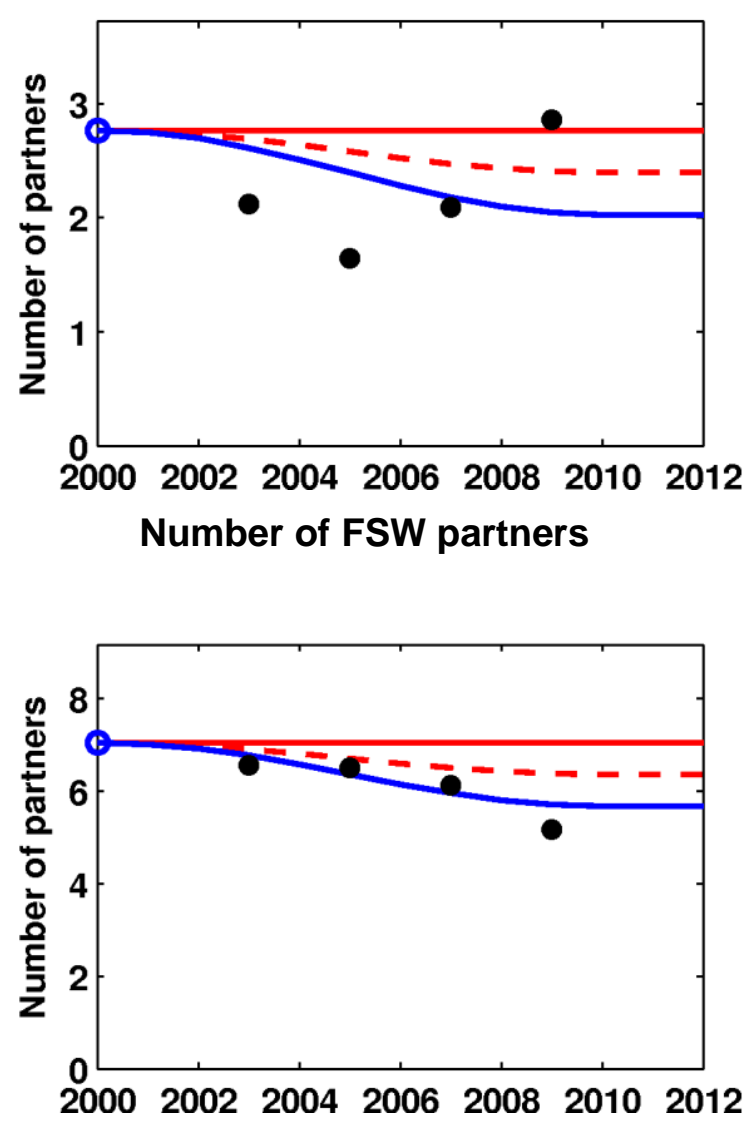

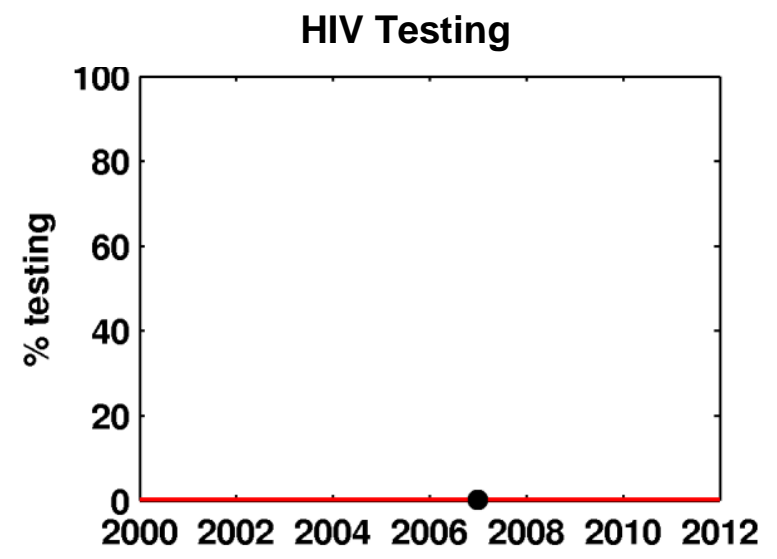


Condom use with females

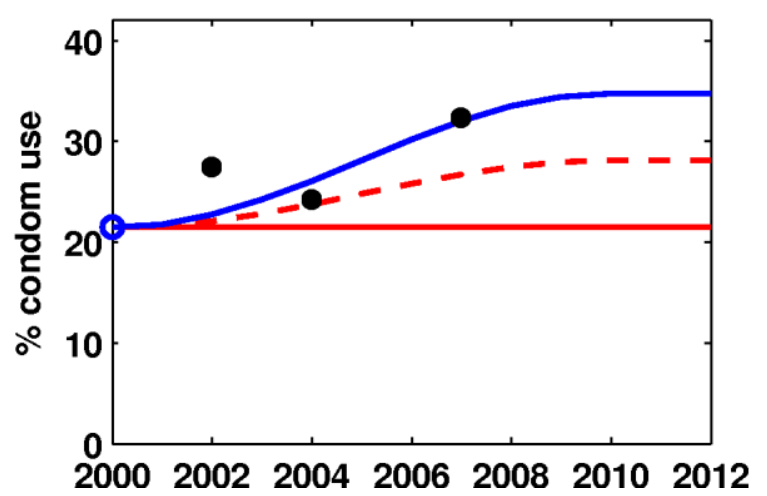

Condom use with Waria
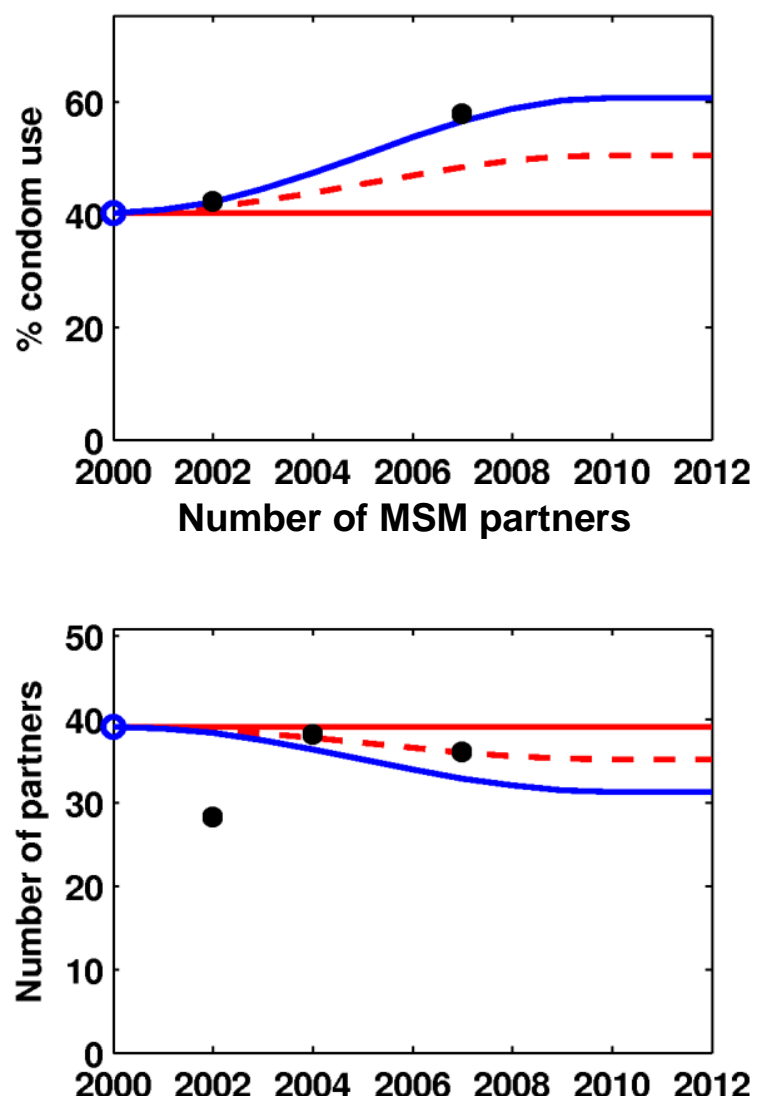

Condom use with other MSM

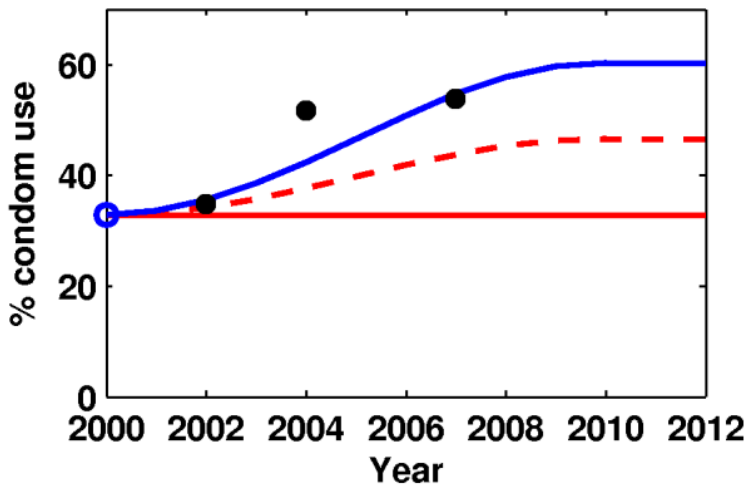

Number of female partners
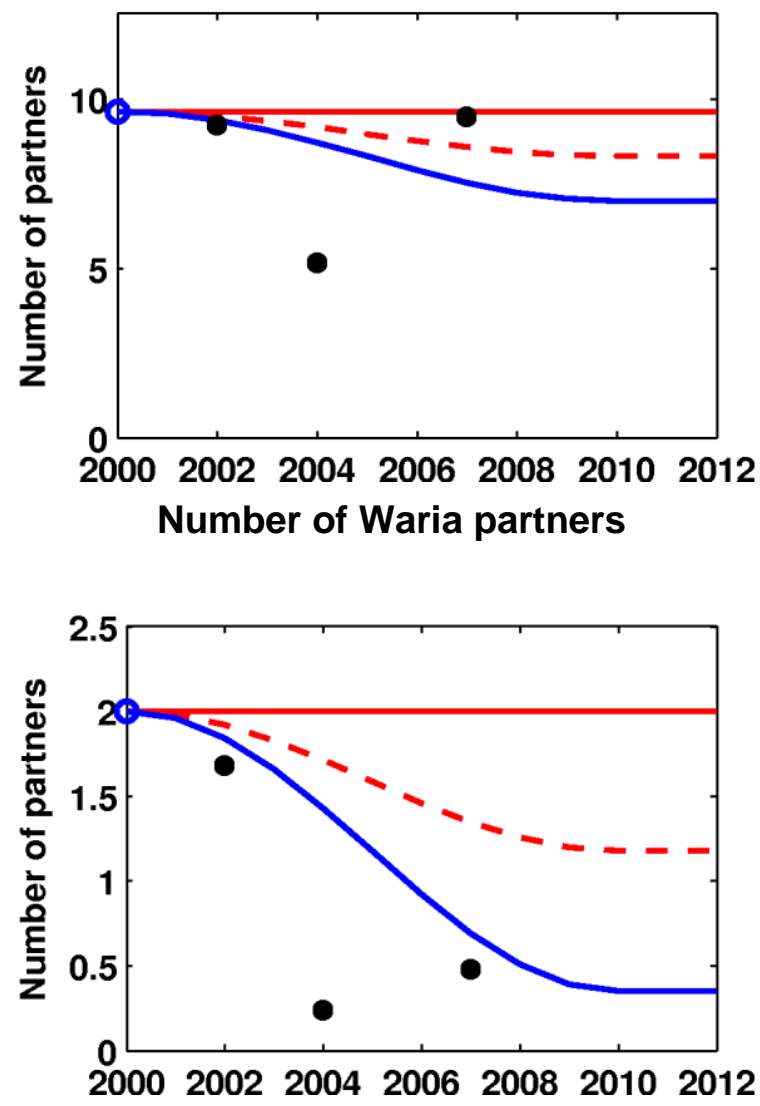
HIV testing

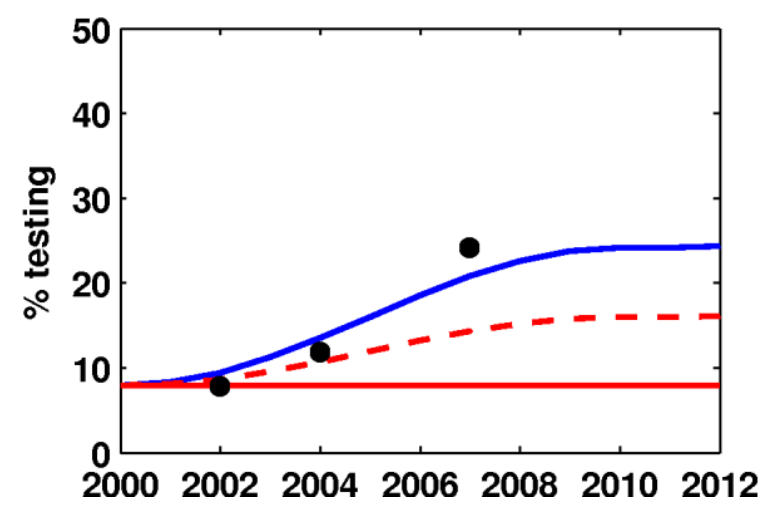

Counterfactuals for Waria programs

Condom use with casual partners
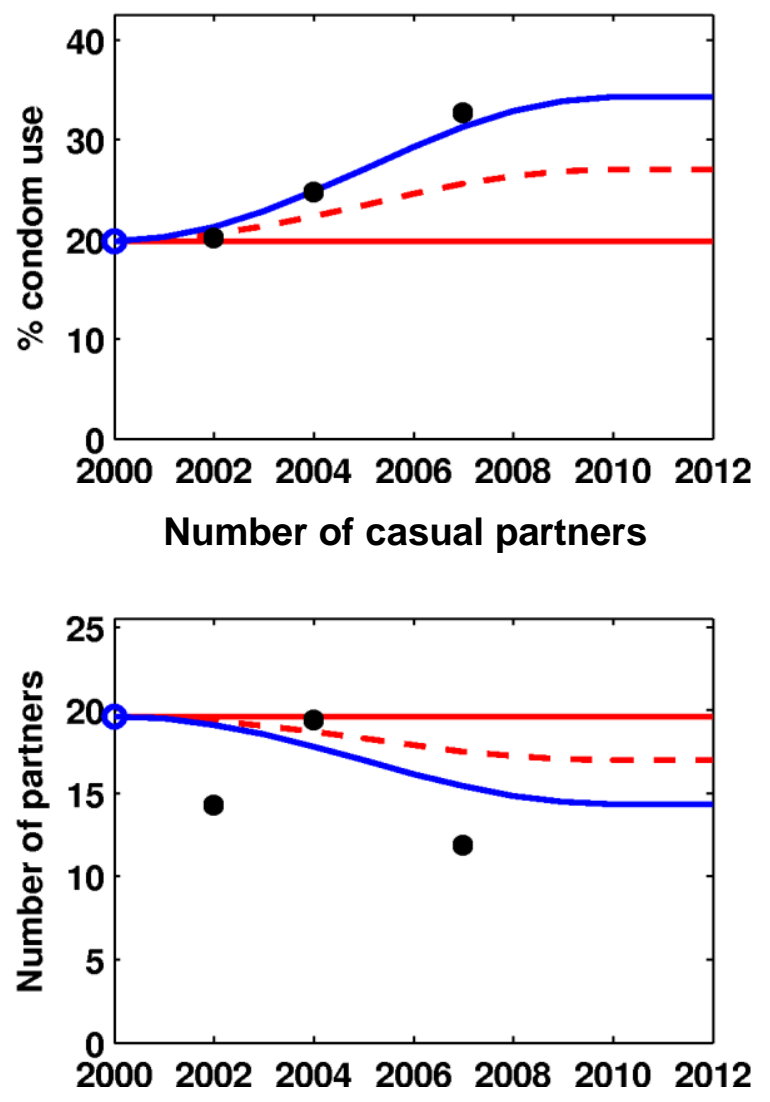

Condom use with commercial partners
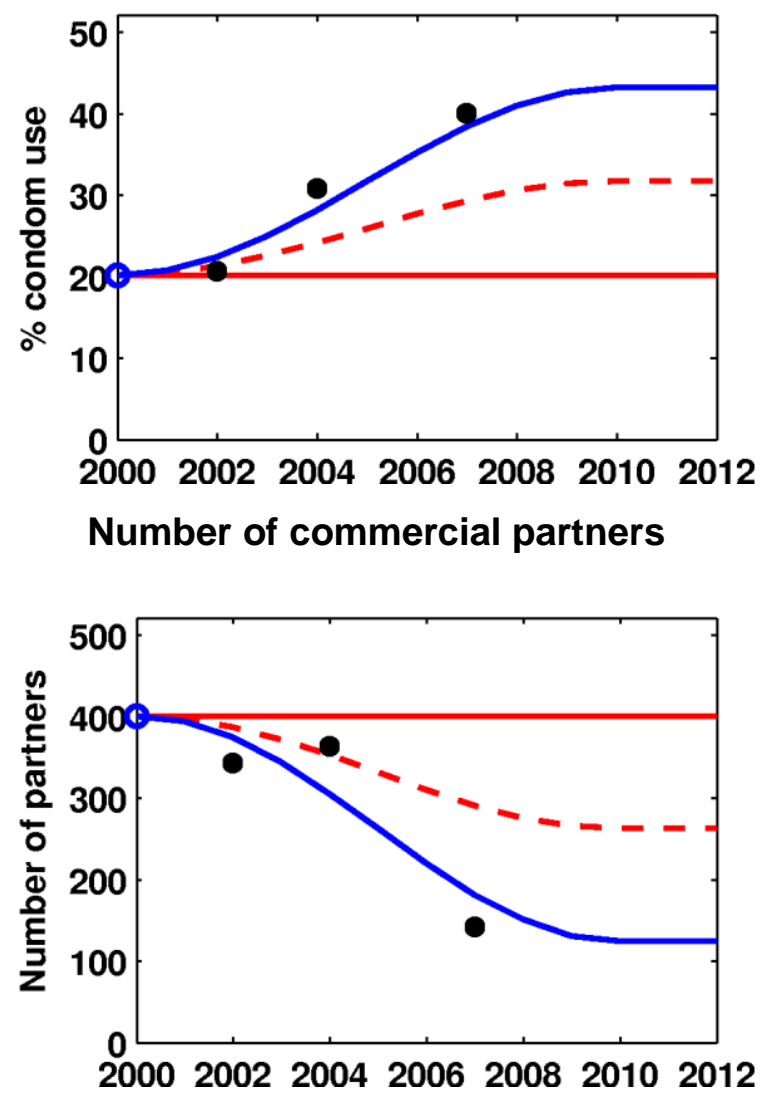

HIV Testing 


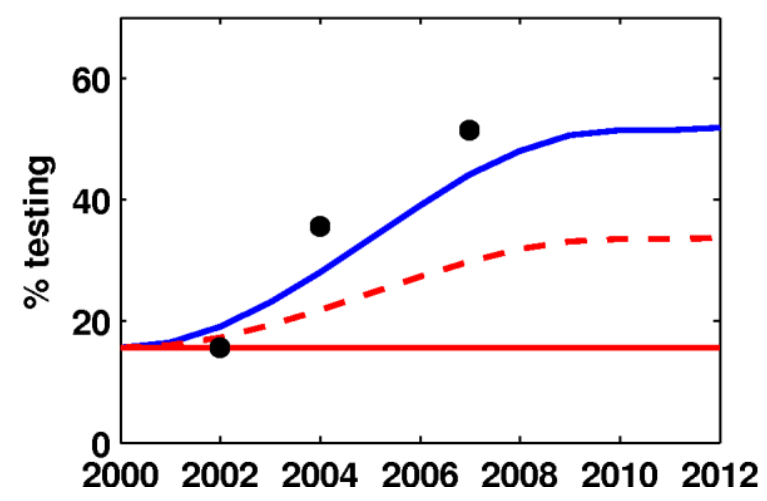

Counterfactuals for IDU needle syringe programs

Percentage of IDUs that share

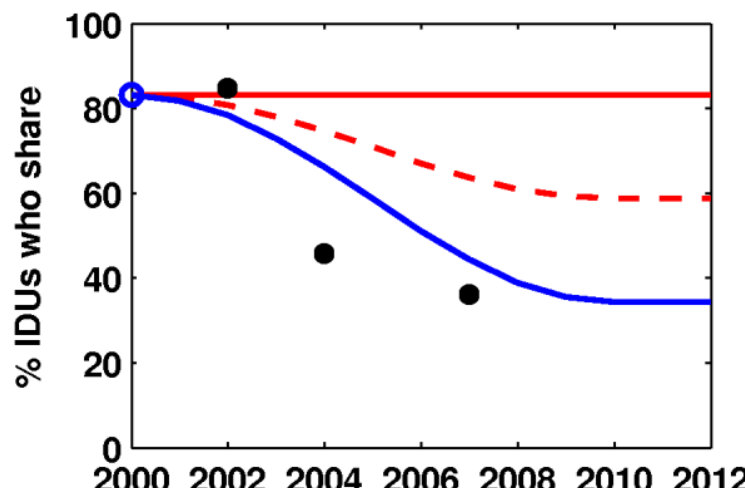

2000200220042006200820102012
Of IDUs that share percentage of injections shared

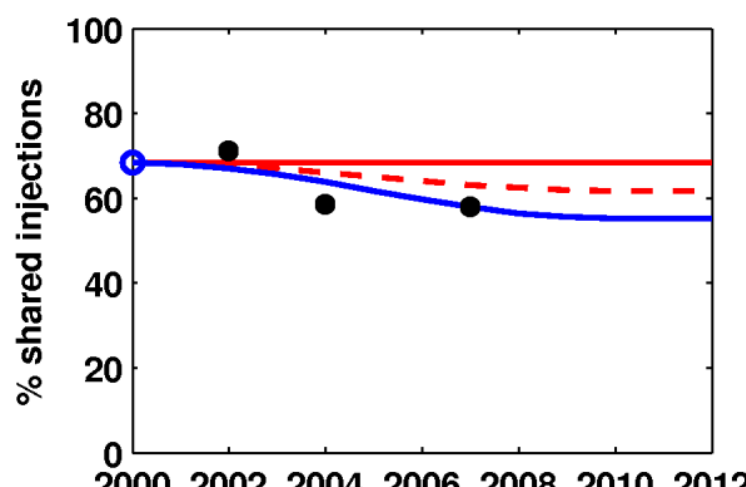

2000200220042006200820102012
Condom use with regular partners

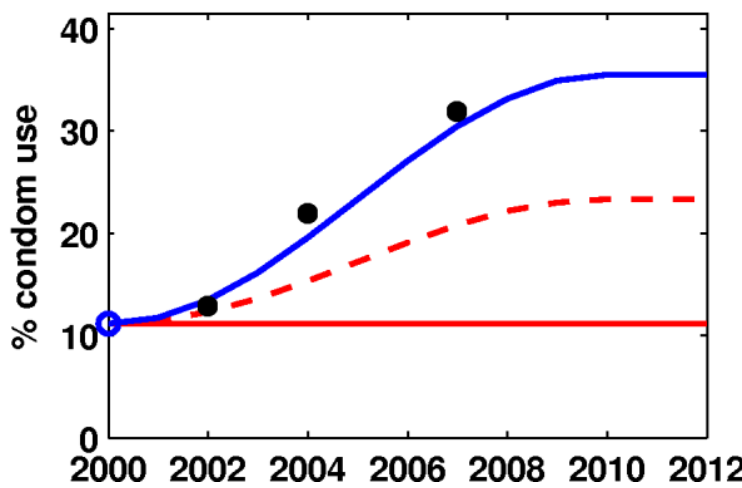

Condom use with casual partners

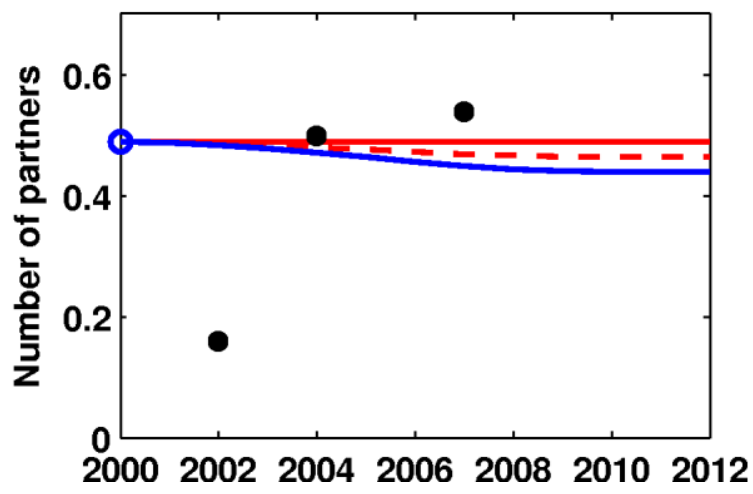

Number of casual partners 


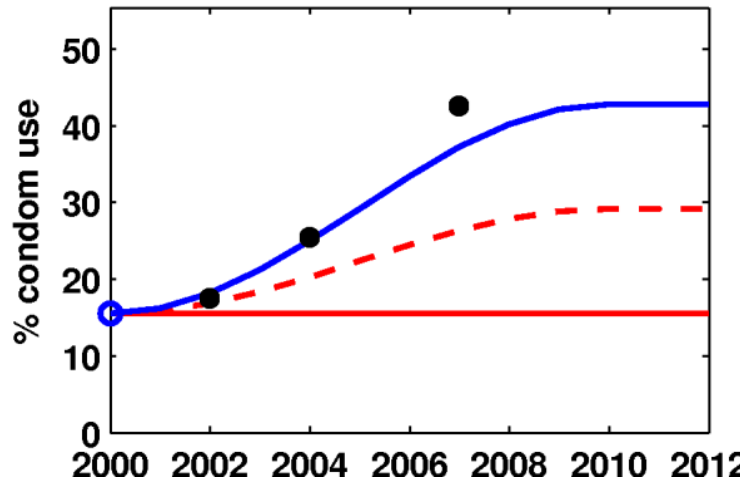

Condom use with commercial partners

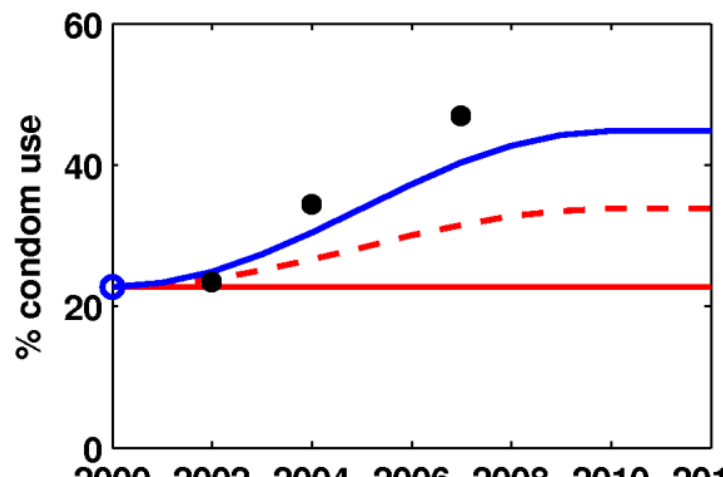

2000200220042006200820102012

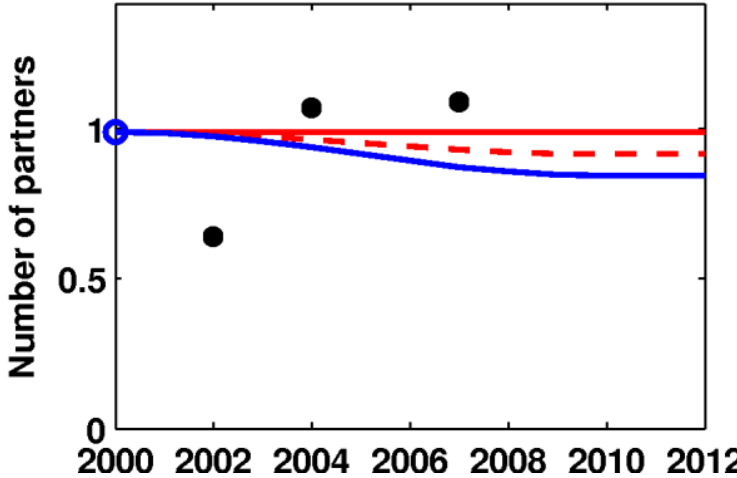

Number of commercial partners

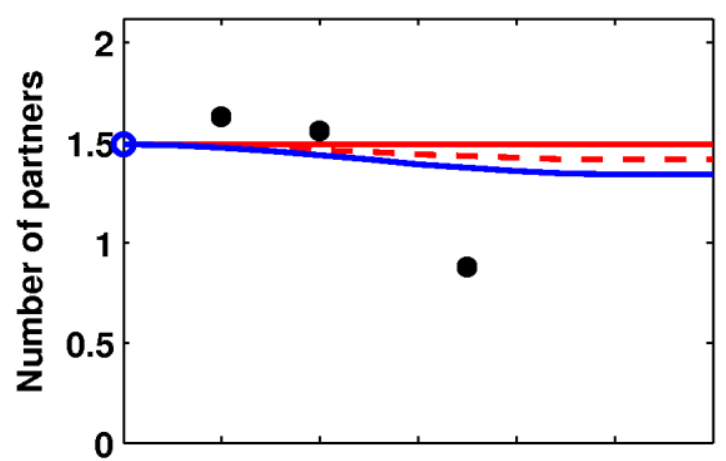

2000200220042006200820102012

HIV Testing

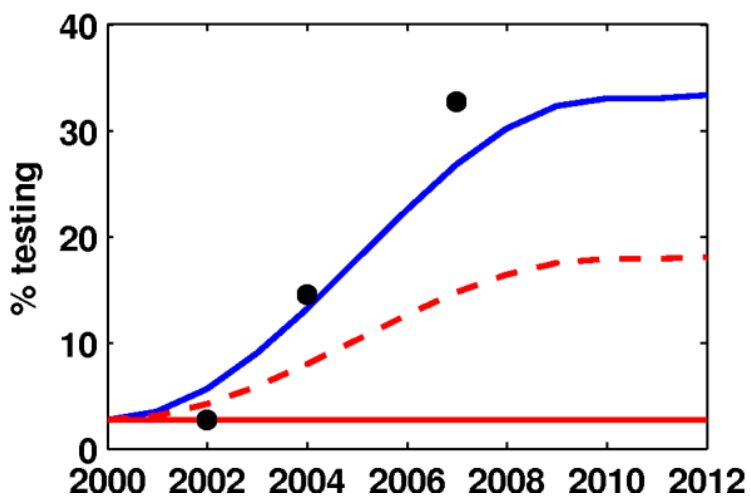


Number of injections

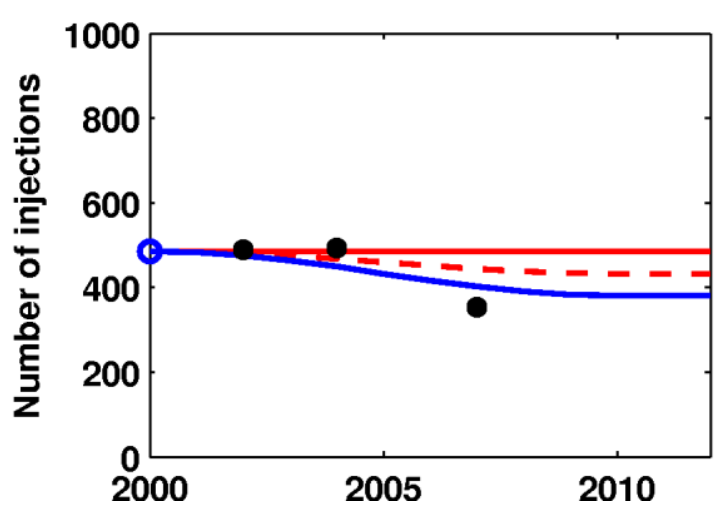

Percentage on MMT

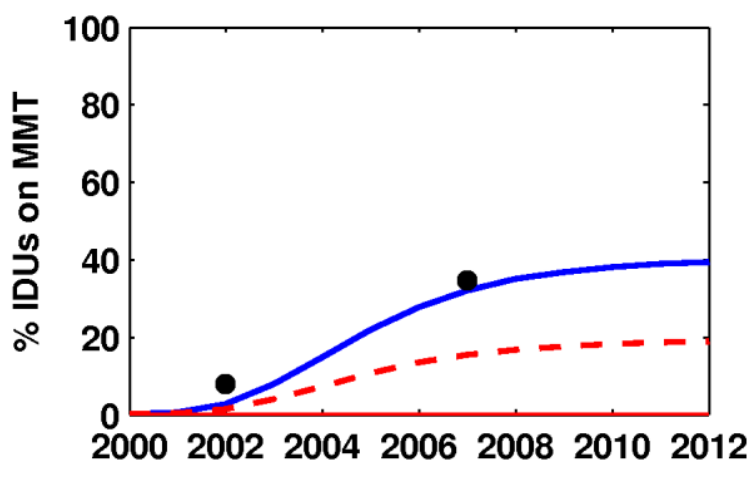

\section{Papua}

Counterfactuals for FSW programs

Condom use with regular partners

Condom use with casual partners
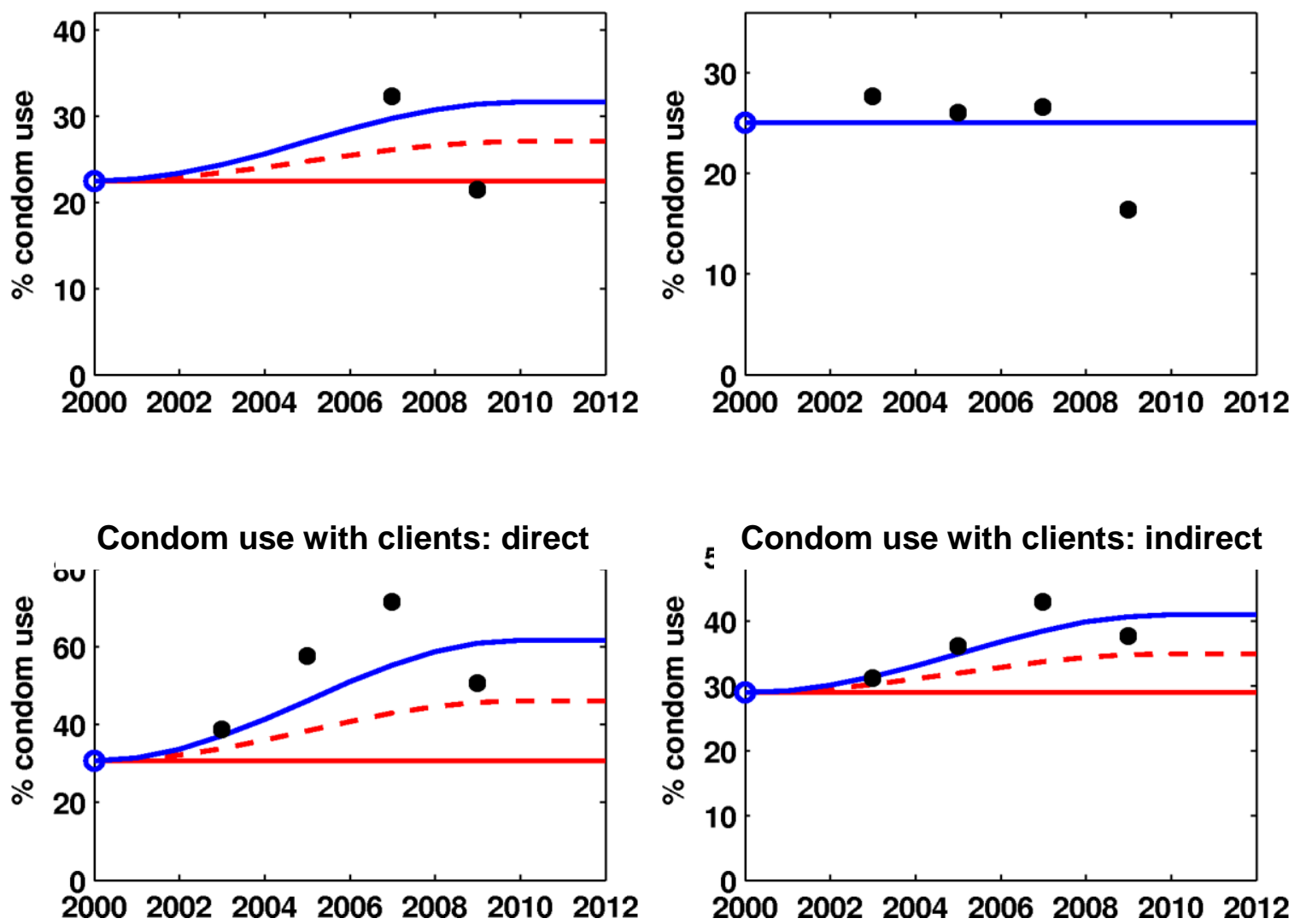

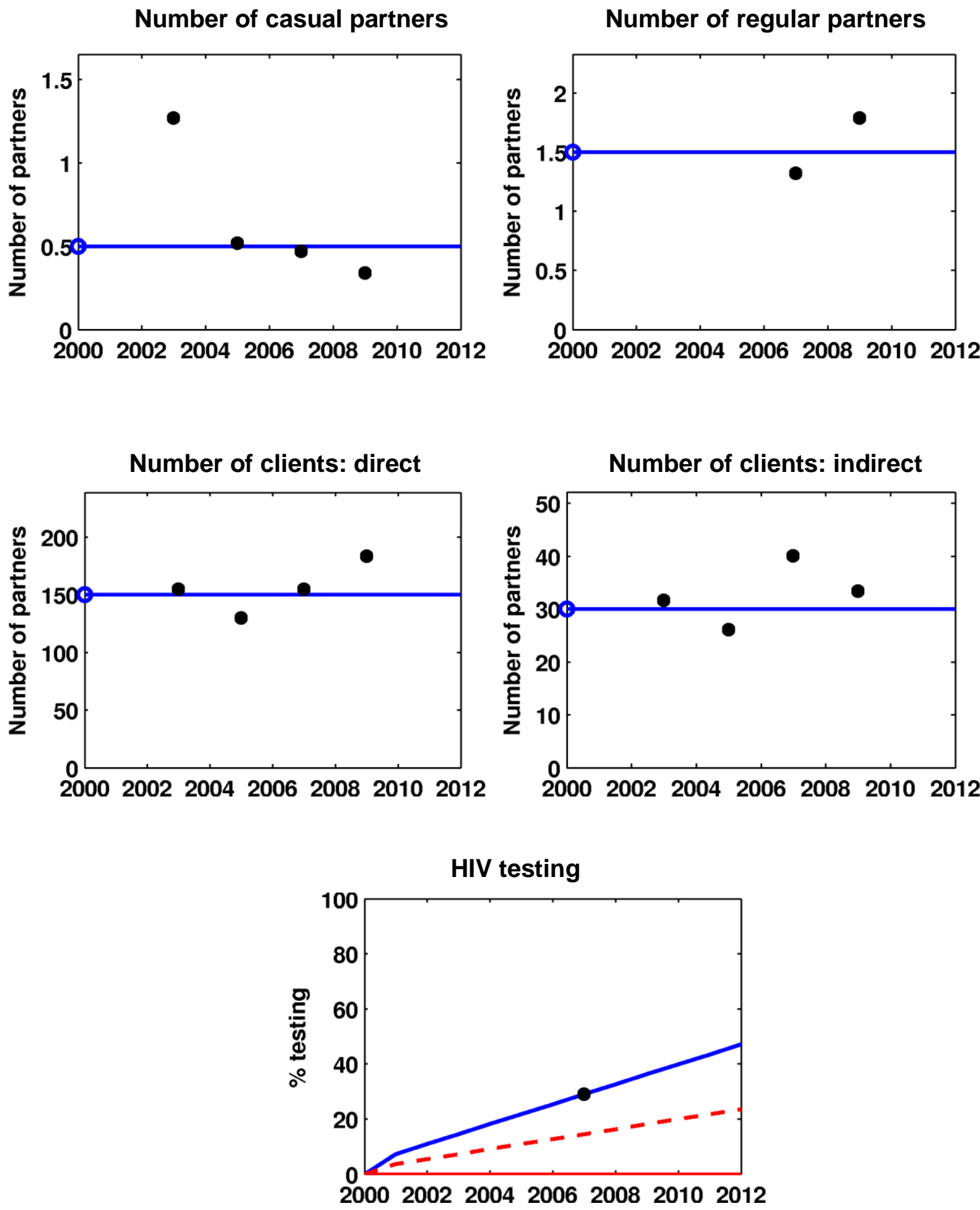


\section{Counterfactuals for Clients programs}

Condom use with casual partners

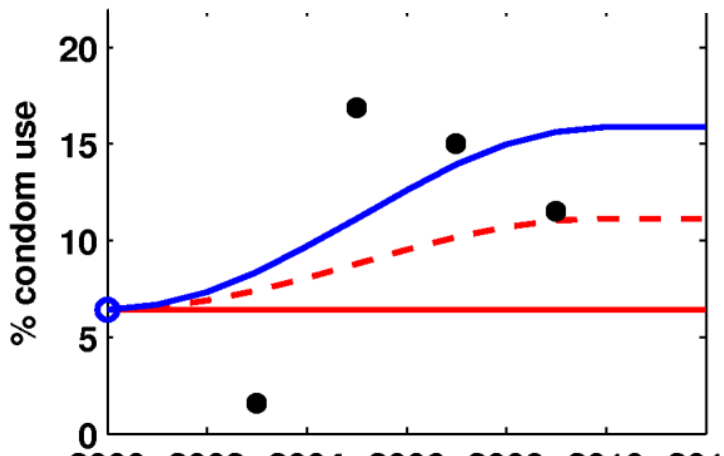

2000200220042006200820102012

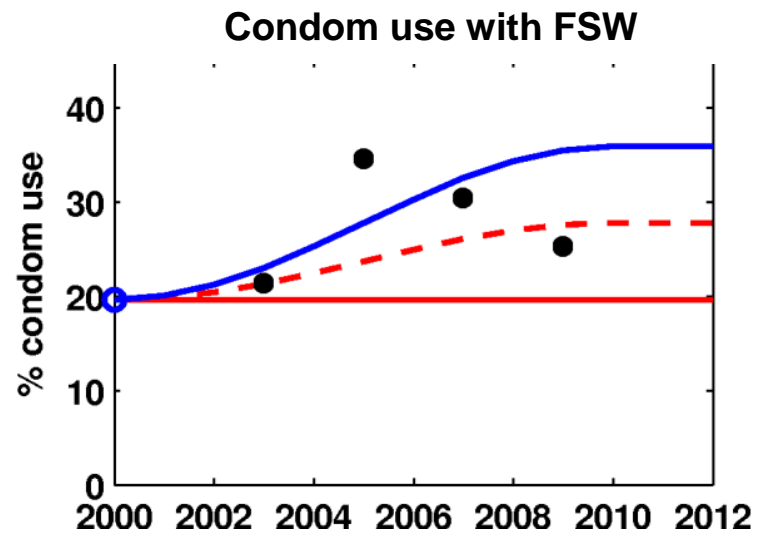

Condom use with regular partners

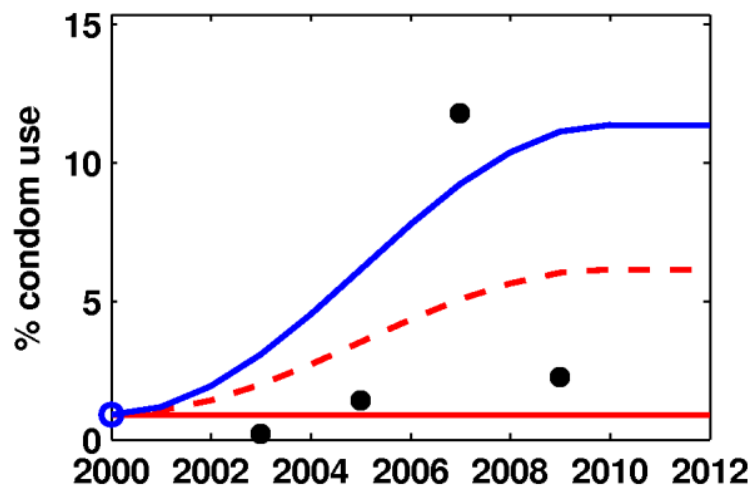

Number of FSW partners

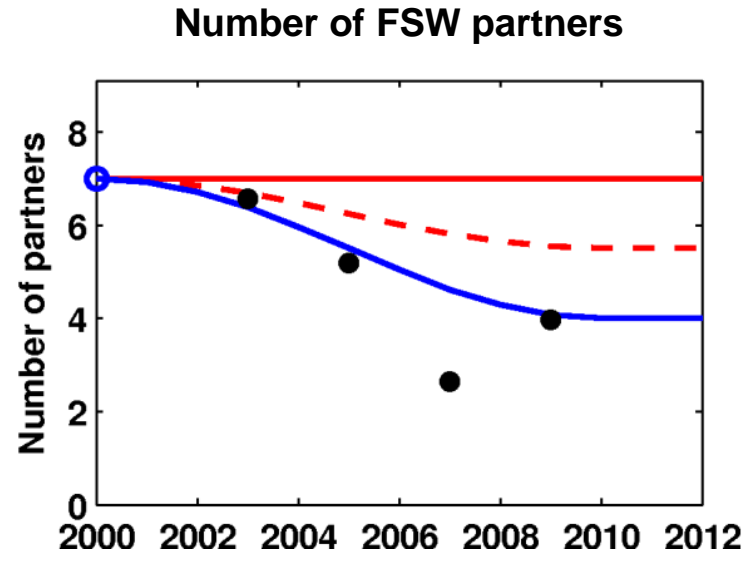

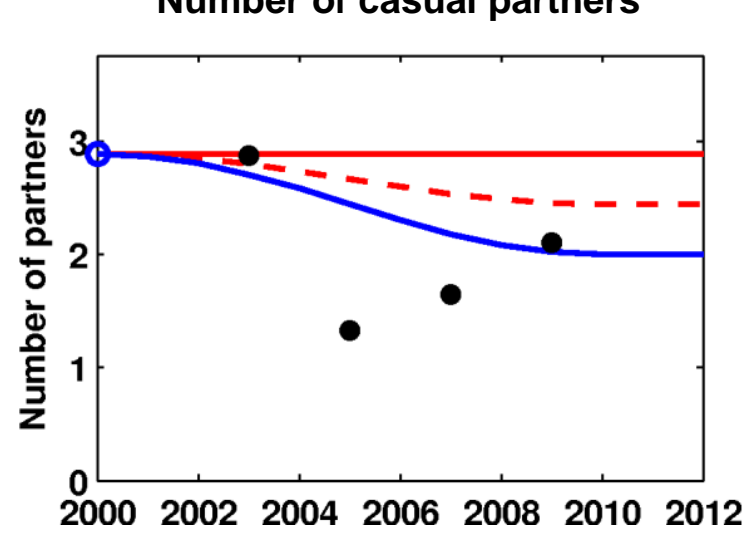

Number of casual partners

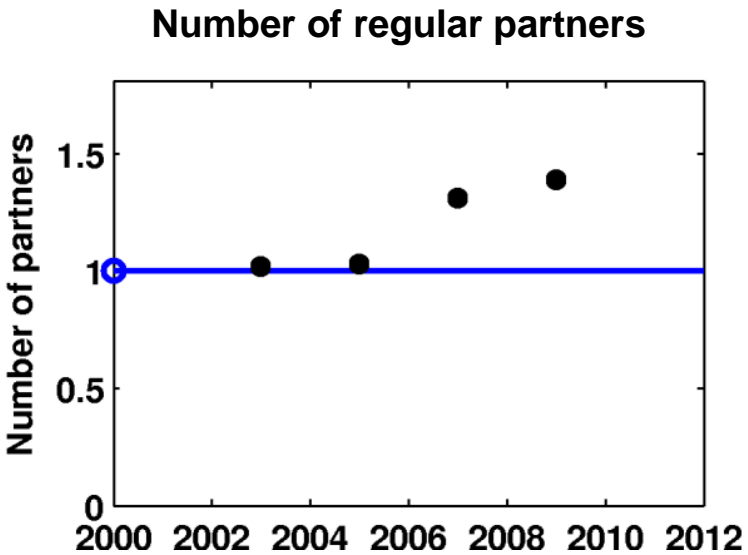


HIV testing

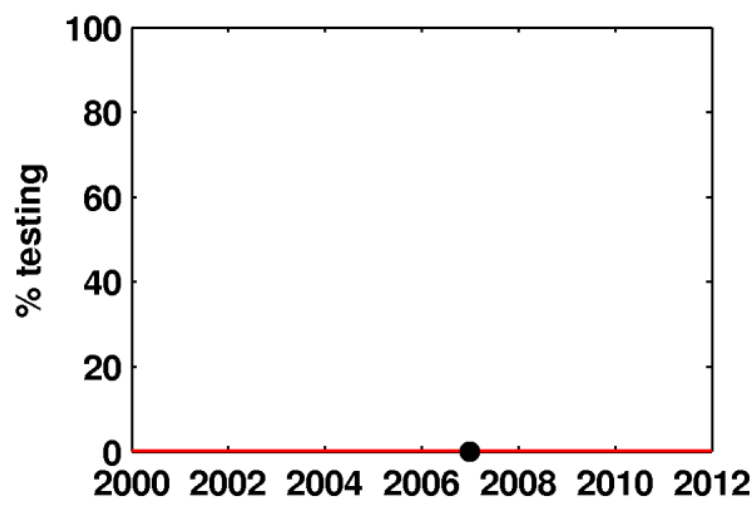

Counterfactuals for low-risk programs

Condom use with regular partners

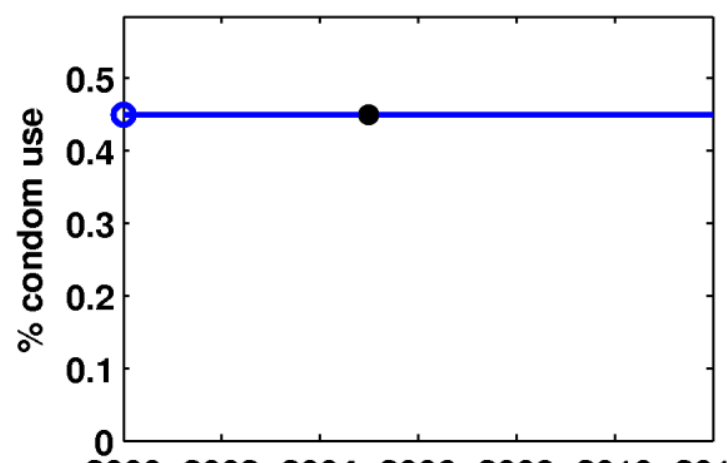

2000200220042006200820102012
Number of regular partners

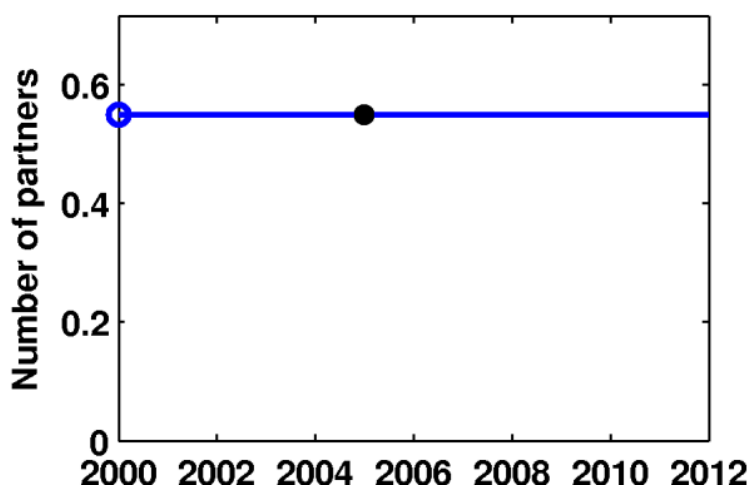

Number of casual partners

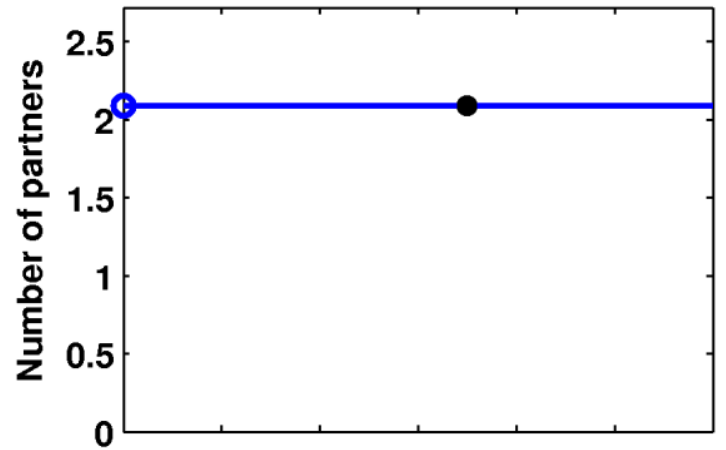

$2000200220042006200820102012 \quad 2000200220042006200820102012$ 


\section{Appendix 6: Optimal Allocation of Future HIV Funding}

To determine the optimal allocation of resources to HIV programs we performed a simple parameter space search. The dimensions of this parameter space represent the proportion of total funding allocated to each HIV prevention program and ART for 2010-2019. We sampled this parameter space a total of 11,000 times and ran HIM on each of the resulting parameter sets for the years 2010 to 2019. The spending proportions that corresponded to the minimum cumulative incidence, minimum number of HIV/AIDS deaths or the maximum number of QALYS gained are then determined. These proportions correspond to the optimal allocation.

To incorporate treatment as prevention we considered three scenarios. For each of these scenarios the testing and treatment rates were changed after 2010 such that the number of people on ART matched the available funding allocation for ART. The first scenario increased the rate that diagnosed infected people with CD4 < 350 begin treatment. The second scenario changed the ART initiation rate for all diagnosed people; this rate was assumed to be equal for all CD4 levels. The final scenario doubled the testing rate of the population and increased the rate that diagnosed people begin ART. 